Supporting information for

\title{
Sesquiterpenoids from Cultures of the Basidiomycetes Irpex lacteus
}

Meng Wang,${ }^{\dagger}$ Jiao-Xian Du, ${ }^{\dagger}$ Hui-Xiang Yang,,${ }^{\dagger}$ Quan Dai,${ }^{\dagger}$ Ya-Pei Liu,${ }^{\dagger}$ Juan He,${ }^{\dagger}$ Yi

Wang, ${ }^{\ddagger}$ Zheng-Hui Li ${ }^{\dagger}{ }^{\top}$ Tao Feng, ${ }^{*}, \dagger, \S$ and Ji-Kai Liu ${ }^{*}, \dagger$

†School of Pharmaceutical Sciences, South-Central University for Nationalities, Wuhan 430074,

People's Republic of China

${ }^{\S}$ Institute for Organic Chemistry and Centre for Biomolecular Drug Research, Leibniz University

Hannover, Hannover 30167, Germany

${ }^{*}$ Bristol-Myers Squibb, Lawrenceville, New Jersey 08648, USA

Corresponding authors: tfeng @mail.scuec.edu.cn (T. Feng); liujikai @mail.scuec.edu.cn (J.-K. Liu)

Content

Section S1. ECD calculations for 1, 2, and 7

Section S2. Results of immunosuppressive activities assay

Section S3. Results of cytotoxicities

Section S4. NMR, HRESIMS, and(or) CD spectra for tremutins A-H (1-8) 
Section S1. ECD calculations for 1, 2, and 7

\section{S1.1. Computational details for compound 1 (ECD)}<smiles>Cc1ccc2c(c1)[C@H]1COC(=O)[C@@H]1[C@H](O)C[C@H]2C</smiles>

$1 \mathrm{a}$<smiles>Cc1ccc2c(c1)[C@H]1COC(=O)[C@H]1[C@H](O)C[C@H]2O</smiles>

$1 \mathrm{~b}$

Conformation search based on molecular mechanics with MMFF94S force fields were performed for 1a and 1b gave 1 and 1 stable conformers, respectively. All these conformers were further optimized by the density functional theory method at the B3LYP/6-31G(d) level by Gaussian 16 program package. The ECD were calculated using density functional theory (TDDFT) at B3LYP/6-31+G(d) level in methanol with IEFPCM model. The calculated ECD curves were all generated using SpecDis 1.71 with $\sigma=0.35 \mathrm{eV}$, and UV shift $-25 \mathrm{~nm}$, respectively.

Figure S1. Comparison of the calculated ECD spectra for $\mathbf{1 a}$ and $\mathbf{1 b}$ with the experimental spectrum of $\mathbf{1}$ in methanol with PCM model.

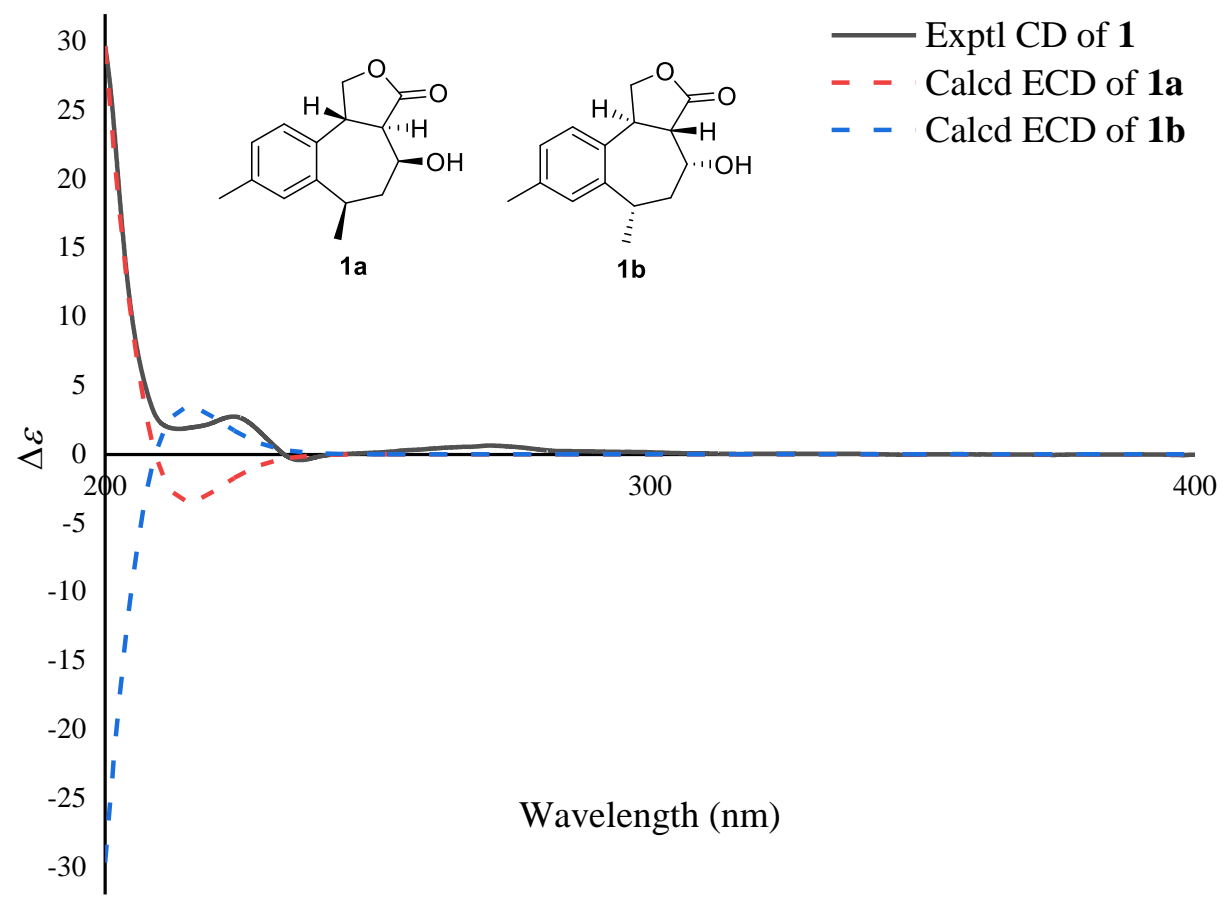


Table S1. Energy analysis for conformers of $\mathbf{1 a A}$ at B3LYP/6-31G(d) level in the gas phase

\begin{tabular}{llllllrr}
\hline Species & $E^{\prime}=E+Z P E$ & $E$ & $H$ & $G$ & $\Delta G$ & $\Delta E(\mathrm{kcal} / \mathrm{mol})$ & $P E \%$ \\
\hline 1aA & -807.824669 & -807.808580 & -807.807636 & -807.867919 & 0 & 0 & $100 \%$ \\
\hline
\end{tabular}

$E, E^{\prime}, H, G$ : total energy, total energy with zero point energy (ZPE), enthalpy, and Gibbs free energy

Figure S2. Main conformers of $\mathbf{1 a}$.

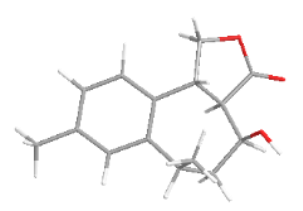

$1 \mathbf{a A}$

Table S2. Energy analysis for conformers of $\boldsymbol{1 b A}$ at B3LYP/6-31G(d) level in the gas phase

\begin{tabular}{llllllrl}
\hline Species & $E^{\prime}=E+Z P E$ & $E$ & $H$ & $G$ & $\Delta G$ & $\Delta E(\mathrm{kcal} / \mathrm{mol})$ & $P E \%$ \\
\hline 1bA & -807.824669 & -807.808580 & -807.807636 & -807.867919 & 0 & 0 & $100 \%$ \\
\hline
\end{tabular}

Figure S3. Main conformers of $\mathbf{1 b}$.

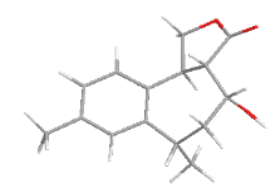

1bA 


\section{S1.2. Computational details for compound 2 (ECD)}<smiles>Cc1cc2c(cc1O)C1=C(CC[Ge](C)(C)C1)C(=O)OC2</smiles>

2a<smiles>Cc1cc2c(cc1O)C1=C(CC[C@H](C)C1)C(=O)OC2</smiles>

$2 b$

Conformation search based on molecular mechanics with MMFF94S force fields were performed for $\mathbf{2 a}$ and $\mathbf{2 b}$ gave 3 and 3 stable conformers, respectively. All these conformers were further optimized on the B3LYP/6-31G(d) level of density functional theory method by Gaussian 16 program package. The ECD were calculated using B3LYP/6-31+G(d) level of theory with IEFPCM solvent model $(\mathrm{MeOH})$. The calculated ECD curves and weighted ECD were all generated using SpecDis 1.71 with $\sigma=0.20 \mathrm{eV}$, and UV shift $3 \mathrm{~nm}$, respectively.

Figure S4. Comparison of the calculated ECD spectra for $\mathbf{2 a}$ and $\mathbf{2 b}$ with the experimental spectrum of $\mathbf{2}$ in methanol with PCM model.

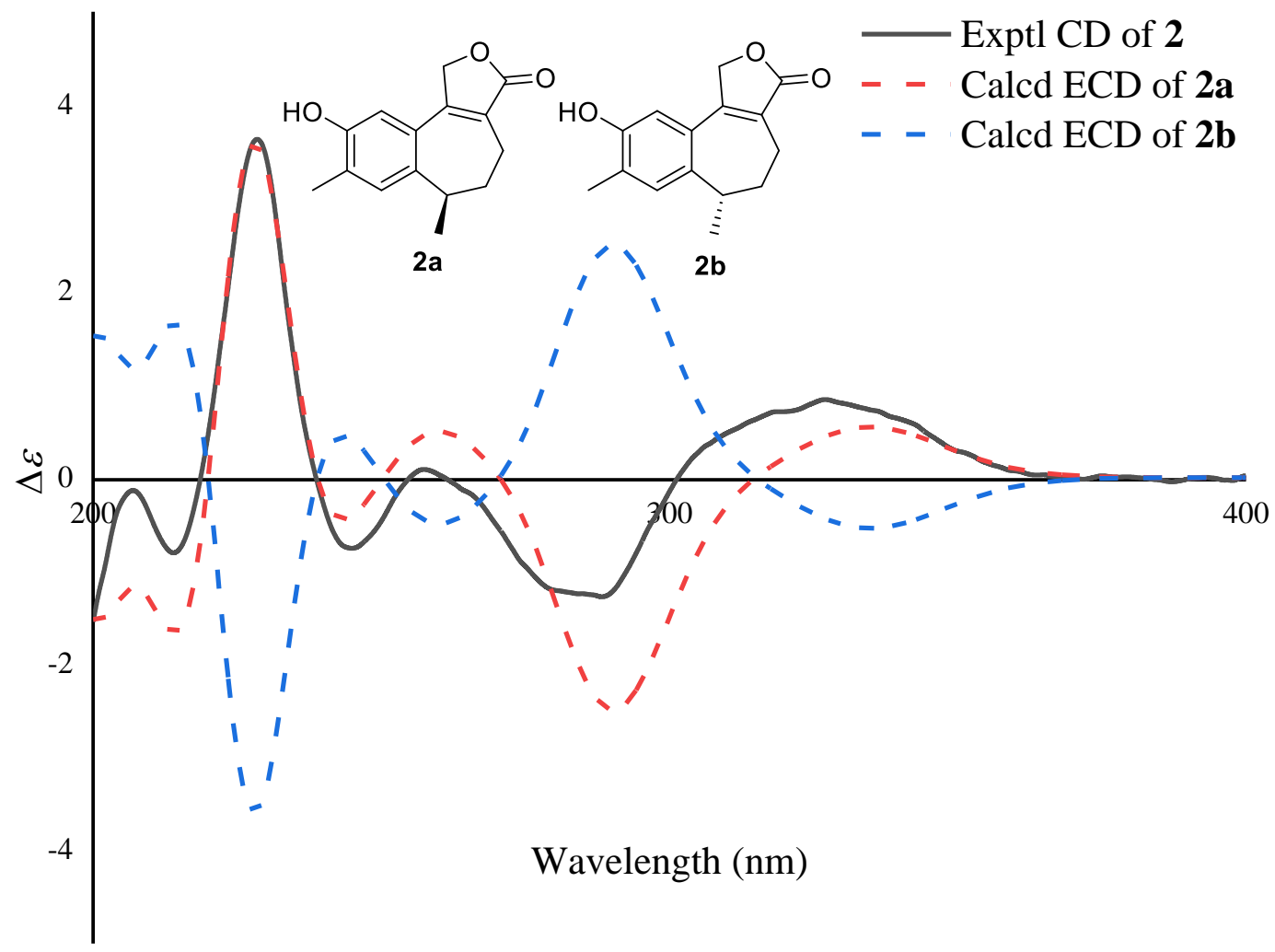


Table S3. Energy analysis for conformers of $\mathbf{2 a A - 2 a C}$ at B3LYP/6-31G(d) level in the gas phase

\begin{tabular}{llllllrl}
\hline Species & $E^{\prime}=E+Z P E$ & $E$ & $H$ & $G$ & $\Delta G$ & $\Delta E(\mathrm{kcal} / \mathrm{mol})$ & \multicolumn{1}{l}{$P E \%$} \\
\hline $\mathbf{2 a A}$ & -806.650624 & -806.634836 & -806.633892 & -806.692957 & 0.000631 & 0.395958 & $31.94 \%$ \\
$\mathbf{2 a B}$ & -806.651293 & -806.635569 & -806.634625 & -806.693588 & 0 & 0 & $62.34 \%$ \\
$\mathbf{2 a C}$ & -806.649037 & -806.633297 & -806.632353 & -806.691334 & 0.002254 & 1.414406 & $5.72 \%$ \\
\hline
\end{tabular}

$E, E^{\prime}, H, G$ : total energy, total energy with zero point energy (ZPE), enthalpy, and Gibbs free energy

Figure S5. Main conformers of 2 a.

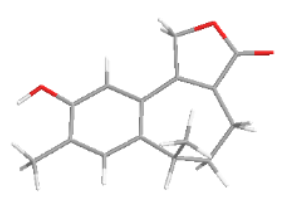

$2 \mathbf{a A}$

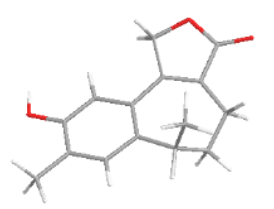

$2 \mathrm{aB}$

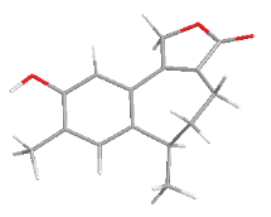

$2 \mathrm{aC}$

Table S4. Energy analysis for conformers of $\mathbf{2} \boldsymbol{b} \boldsymbol{A} \boldsymbol{-}-\mathbf{2} \boldsymbol{b} \boldsymbol{C}$ at B3LYP/6-31G(d) level in the gas phase

\begin{tabular}{llllllrr}
\hline Species & $E^{\prime}=E+Z P E$ & $E$ & $H$ & $G$ & $\Delta G$ & $\Delta E(\mathrm{kcal} / \mathrm{mol})$ & \multicolumn{1}{l}{$P E \%$} \\
\hline 2bA & -806.650624 & -806.634836 & -806.633892 & -806.692957 & 0.000631 & 0.395958 & $31.94 \%$ \\
2bB & -806.651293 & -806.635569 & -806.634625 & -806.693588 & 0 & 0 & $62.34 \%$ \\
2bC & -806.649037 & -806.633297 & -806.632353 & -806.691334 & 0.002254 & 1.414406 & $5.72 \%$ \\
\hline
\end{tabular}

Figure S6. Main conformers for $\mathbf{2 b}$.

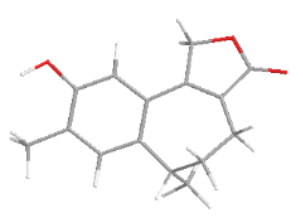

2bA

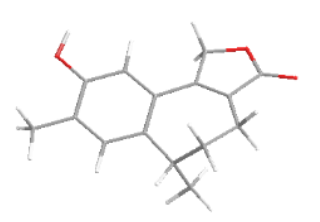

$2 \mathbf{b B}$

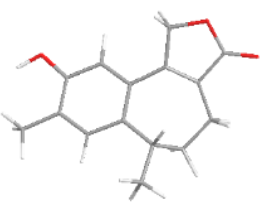

$2 \mathrm{bC}$ 


\section{S1.3. Computational details for compound 7 (ECD)}

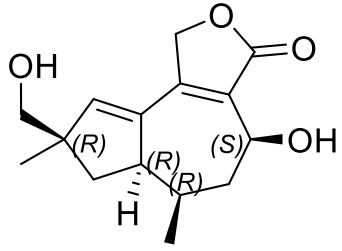

$7 a$

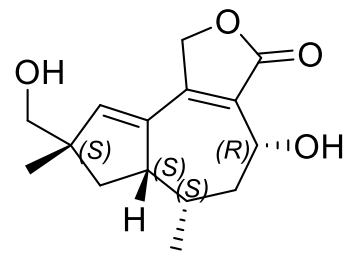

$7 b$

Conformation search based on molecular mechanics with MMFF94S force fields were performed for 7a and 7b gave 8 and 8 stable conformers, respectively. All these conformers were further optimized by the density functional theory method at the B3LYP/6-31G(d) level by Gaussian 16 program package. The ECD were calculated using B3LYP/6-31+G(d) level in methanol with PCM model on B3LYP/6-31+G(d). The calculated ECD curves and weighted ECD were all generated using SpecDis 1.71 with $\sigma=0.30 \mathrm{eV}$, and UV shift $-10 \mathrm{~nm}$, respectively.

Figure S7. Comparison of the calculated ECD spectra for $\mathbf{7 a}$ and $\mathbf{7 b}$ with the experimental spectrum of $\mathbf{7}$ in methanol with PCM model.

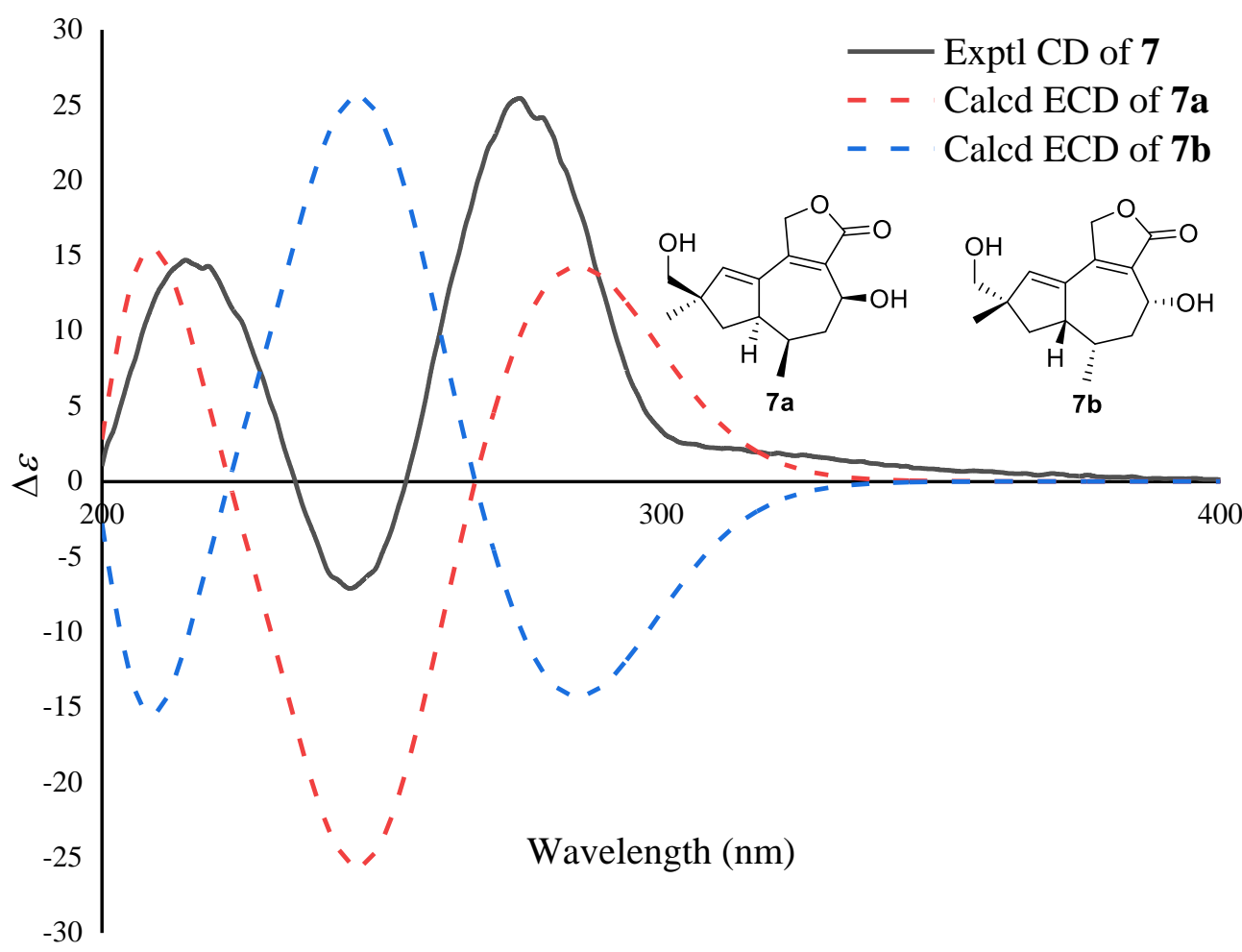


Table S5. Energy analysis for conformers of $7 \boldsymbol{a A}-\mathbf{7 a H}$ at B3LYP/6-31G(d) level in the gas phase

\begin{tabular}{llllllrr}
\hline Species & $E^{\prime}=E+Z P E$ & $E$ & $H$ & $G$ & $\Delta G$ & $\Delta E(\mathrm{kcal} / \mathrm{mol})$ & \multicolumn{1}{l}{$P E \%$} \\
\hline $\mathbf{7 a A}$ & -884.188699 & -884.170778 & -884.169833 & -884.233645 & 0.000464 & 0.291164 & $25.11 \%$ \\
$\mathbf{7 a B}$ & -884.188844 & -884.170953 & -884.170008 & -884.233926 & 0.000183 & 0.114834 & $33.82 \%$ \\
$\mathbf{7 a C}$ & -884.189250 & -884.171462 & -884.170518 & -884.234109 & 0 & 0 & $41.06 \%$ \\
$\mathbf{7 a D}$ & -884.188178 & -884.170210 & -884.169266 & -884.233312 & 0.000797 & 0.500125 & $17.64 \%$ \\
$\mathbf{7 a E}$ & -884.188766 & -884.170891 & -884.169947 & -884.233440 & 0.000669 & 0.419804 & $20.21 \%$ \\
$\mathbf{7 a F}$ & -884.188811 & -884.170900 & -884.169956 & -884.233696 & 0.000413 & 0.259161 & $26.51 \%$ \\
$\mathbf{7 a G}$ & -884.186478 & -884.168647 & -884.167702 & -884.231152 & 0.002957 & 1.855546 & $1.79 \%$ \\
$\mathbf{7 a H}$ & -884.188929 & -884.171110 & -884.170166 & -884.233732 & 0.000377 & 0.236571 & $27.54 \%$ \\
\hline
\end{tabular}

$E, E^{\prime}, H, G$ : total energy, total energy with zero point energy (ZPE), enthalpy, and Gibbs free energy

Figure S8. Main conformers for 7a.

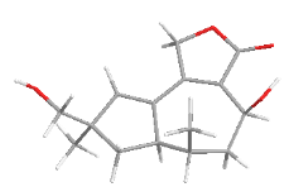

$7 \mathbf{a A}$

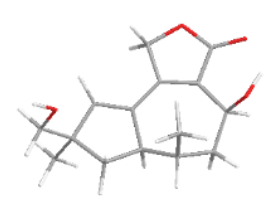

$7 \mathrm{aE}$

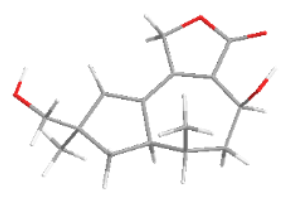

$7 \mathbf{a B}$

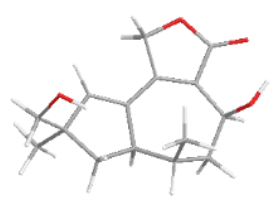

$7 \mathbf{a F}$

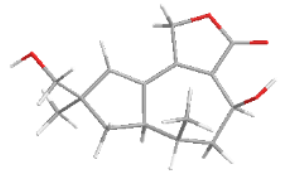

$7 \mathbf{a C}$

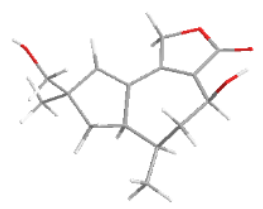

$7 \mathbf{a G}$
$7 \mathrm{aD}$
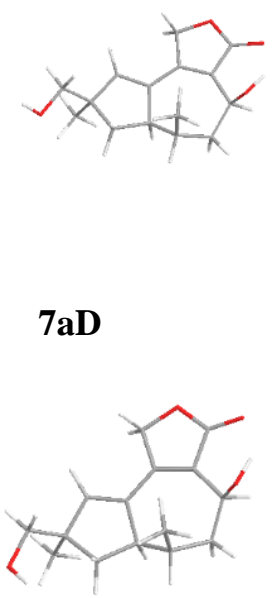

$7 \mathbf{a H}$

Table S6. Energy analysis for conformers of $7 \boldsymbol{b} \boldsymbol{A}-\mathbf{7} \boldsymbol{b} \boldsymbol{H}$ at B3LYP/6-31G(d) level in the gas phase

\begin{tabular}{llllllrl}
\hline Species & $E^{\prime}=E+Z P E$ & $E$ & $H$ & $G$ & $\Delta G$ & $\Delta E(\mathrm{kcal} / \mathrm{mol})$ & \multicolumn{1}{l}{$P E \%$} \\
\hline $\mathbf{7 b A}$ & -884.188699 & -884.170778 & -884.169833 & -884.233645 & 0.000464 & 0.291164 & $25.11 \%$ \\
7bB & -884.188844 & -884.170953 & -884.170008 & -884.233926 & 0.000183 & 0.114834 & $33.82 \%$ \\
7bC & -884.189250 & -884.171462 & -884.170518 & -884.234109 & 0 & 0 & $41.06 \%$ \\
7bD & -884.188178 & -884.170210 & -884.169266 & -884.233312 & 0.000797 & 0.500125 & $17.64 \%$ \\
7bE & -884.188766 & -884.170891 & -884.169947 & -884.233440 & 0.000669 & 0.419804 & $20.21 \%$ \\
7bF & -884.188811 & -884.170900 & -884.169956 & -884.233696 & 0.000413 & 0.259161 & $26.51 \%$ \\
7bG & -884.186478 & -884.168647 & -884.167702 & -884.231152 & 0.002957 & 1.855546 & $1.79 \%$ \\
7bH & -884.188929 & -884.171110 & -884.170166 & -884.233732 & 0.000377 & 0.236571 & $27.54 \%$ \\
\hline
\end{tabular}


Figure S9. Main conformers for $\mathbf{7 b}$.

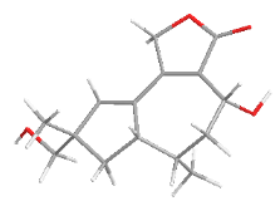

$7 \mathbf{b A}$

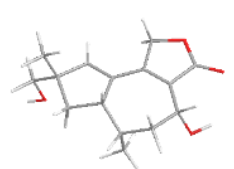

$7 b \mathbf{b}$

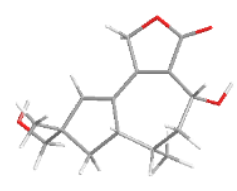

7bB

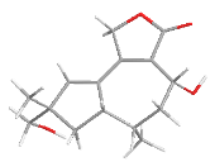

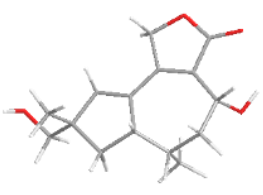

$7 \mathrm{bC}$

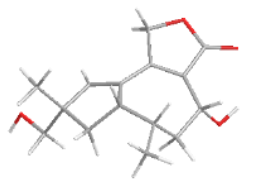

$7 b G$

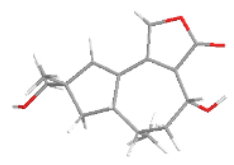

7bD

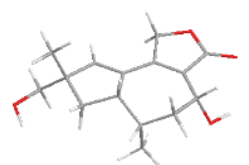

7bH 
Section S2. Results of immunosuppressive activities assay

Table S7. Immunosuppressive activities of $\mathbf{1}$ and $\mathbf{2}^{a}$

\begin{tabular}{|c|c|c|c|c|c|}
\hline \multirow[t]{2}{*}{ Compd } & & \multicolumn{2}{|c|}{$\begin{array}{c}\text { ConA-induced } \\
\text { T-cell } \\
\text { proliferation } \\
\end{array}$} & \multicolumn{2}{|l|}{$\begin{array}{l}\text { LPS-induced } \\
\text { B-cell } \\
\text { proliferation } \\
\end{array}$} \\
\hline & $\begin{array}{l}\mathrm{CC}_{50} \\
(\mu \mathrm{M})\end{array}$ & $\mathrm{IC}_{50}(\mu \mathrm{M})$ & SI & $\mathrm{IC}_{50}(\mu \mathrm{M})$ & $\mathrm{SI}^{\mathrm{a}}$ \\
\hline 1 & 172.3 & $>40$ & $<4.30$ & 22.4 & 7.69 \\
\hline 2 & 115.7 & 26.7 & 4.33 & 13.6 & 8.51 \\
\hline CsA & $>2.50$ & 0.03 & $>83.33$ & 0.32 & $>7.81$ \\
\hline
\end{tabular}

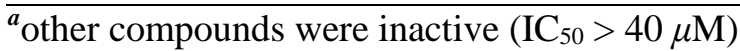




\section{Section S3. Results of cytotoxicities}

Table S8. Cytotoxicities for $\mathbf{6 , 7 , 1 0}$, and $\mathbf{1 1}\left(\mathrm{IC}_{50}, \mu \mathrm{M}\right)^{a}$

\begin{tabular}{cccccc}
\hline No. & HL-60 & SMMC-7721 & SW480 & A-549 & MCF-7 \\
\hline $\mathbf{6}$ & $32.0 \pm 0.81$ & $26.3 \pm 0.77$ & $>40$ & $11.5 \pm 1.13$ & $>40$ \\
$\mathbf{7}$ & $27.6 \pm 0.37$ & $>40$ & $33.3 \pm 0.53$ & $>40 \pm 0.89$ & $24.1 \pm 0.62$ \\
$\mathbf{1 0}$ & $19.3 \pm 0.62$ & $11.7 \pm 0.61$ & $17.7 \pm 0.87$ & $28.9 \pm 1.08$ & $15.4 \pm 1.83$ \\
$\mathbf{1 1}$ & $>40$ & $26.8 \pm 1.31$ & $>40$ & $32.4 \pm 0.43$ & $27.0 \pm 0.91$ \\
Taxol & $<0.008$ & $<0.008$ & $<0.008$ & $<0.008$ & $<0.008$ \\
\hline
\end{tabular}

${ }^{a}$ other compounds were inactive at the concentration of $40 \mu \mathrm{M}$ 
Section S4. NMR, HRESIMS, and(or) CD spectra for tremutins A-H (1-8)

4.1 NMR, HRESIMS, and CD spectra for tremutin A (1)

${ }^{1} \mathrm{H}$ NMR spectrum of $\mathbf{1}$

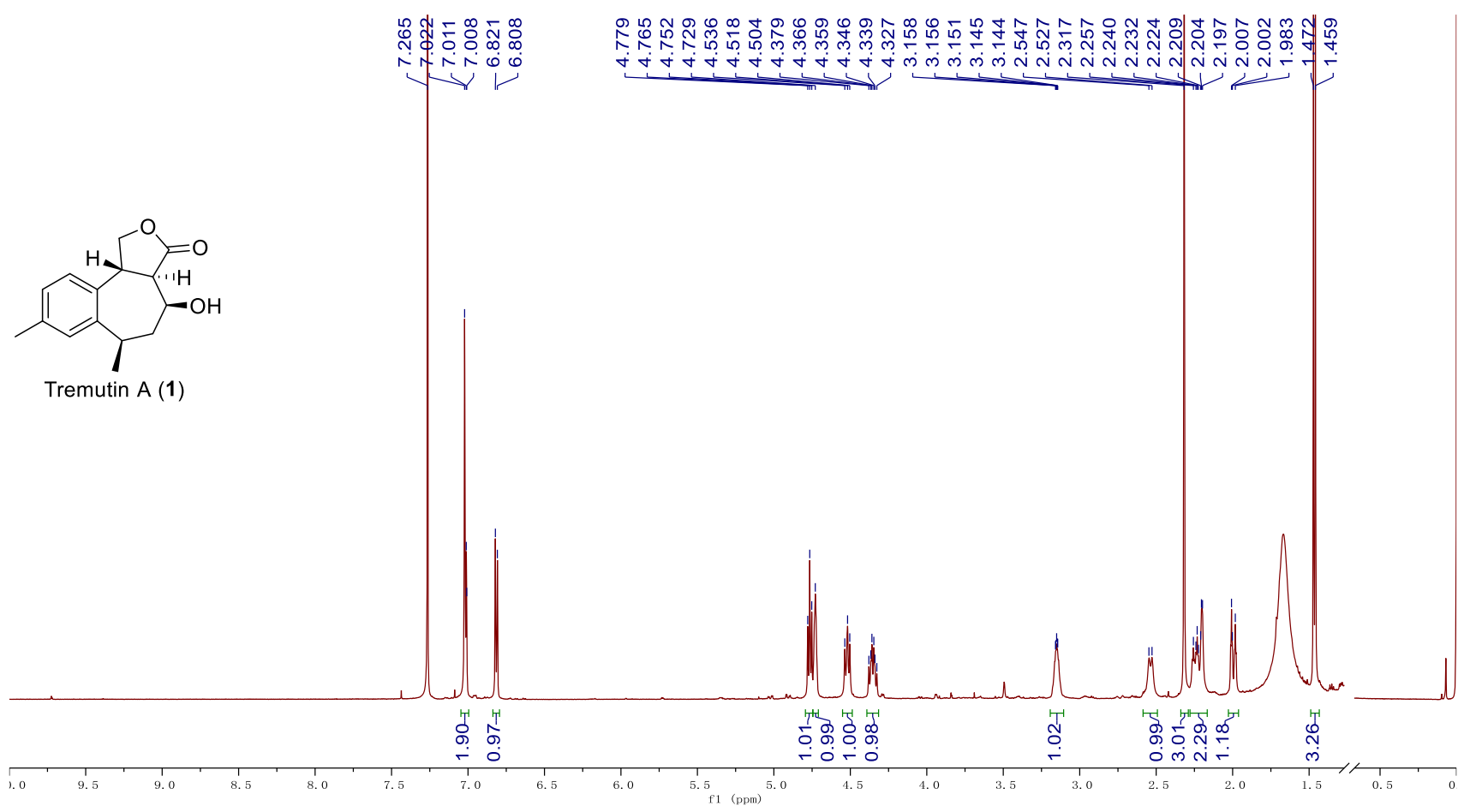

${ }^{13} \mathrm{C}$ NMR and DEPT spectra of $\mathbf{1}$

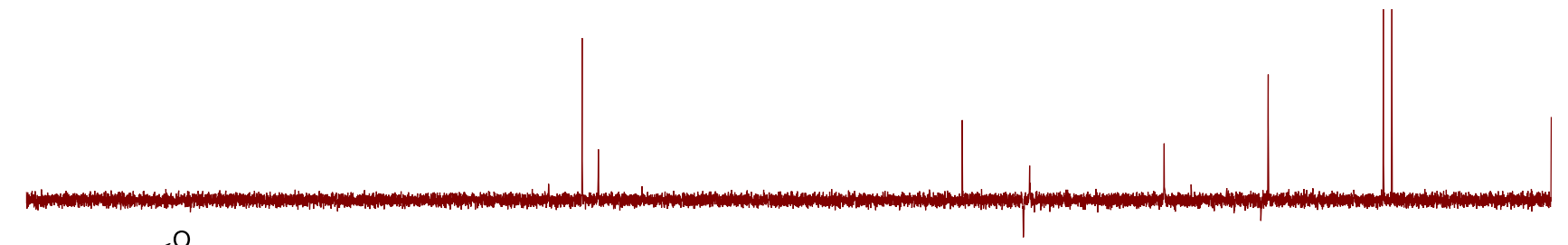<smiles>Cc1ccc2c(c1)C(C)CC(O)[C@H]1C(=O)OC[C@H]21</smiles>

Tremutin A (1)
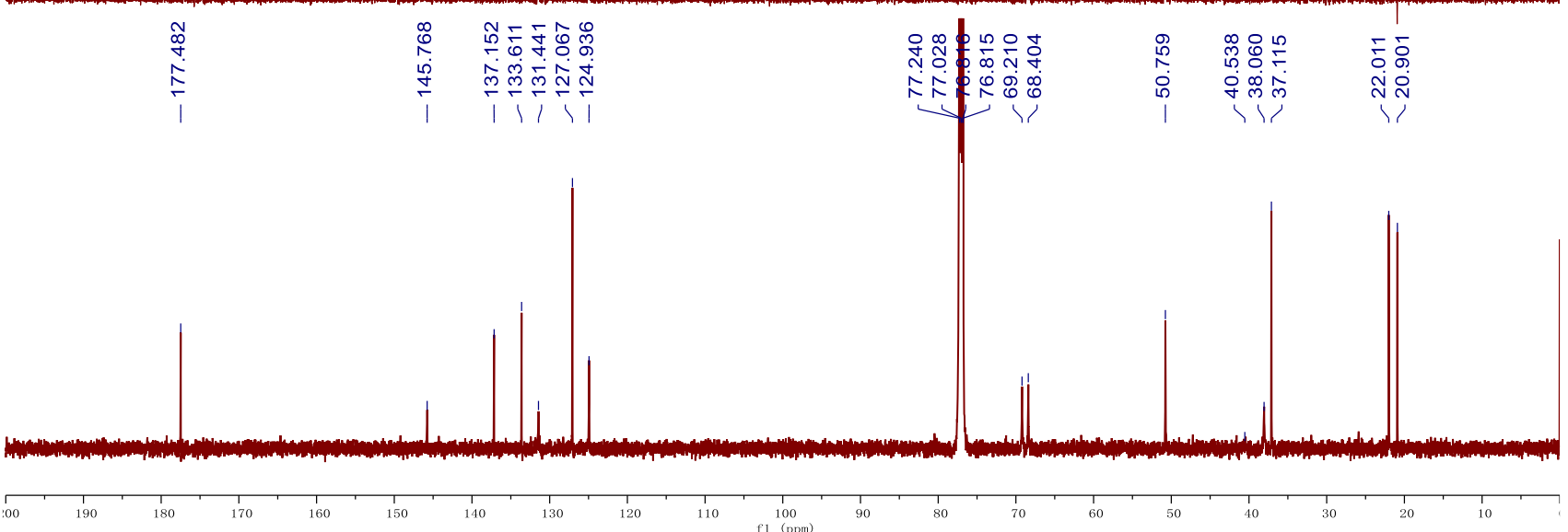
HSQC spectrum of $\mathbf{1}$

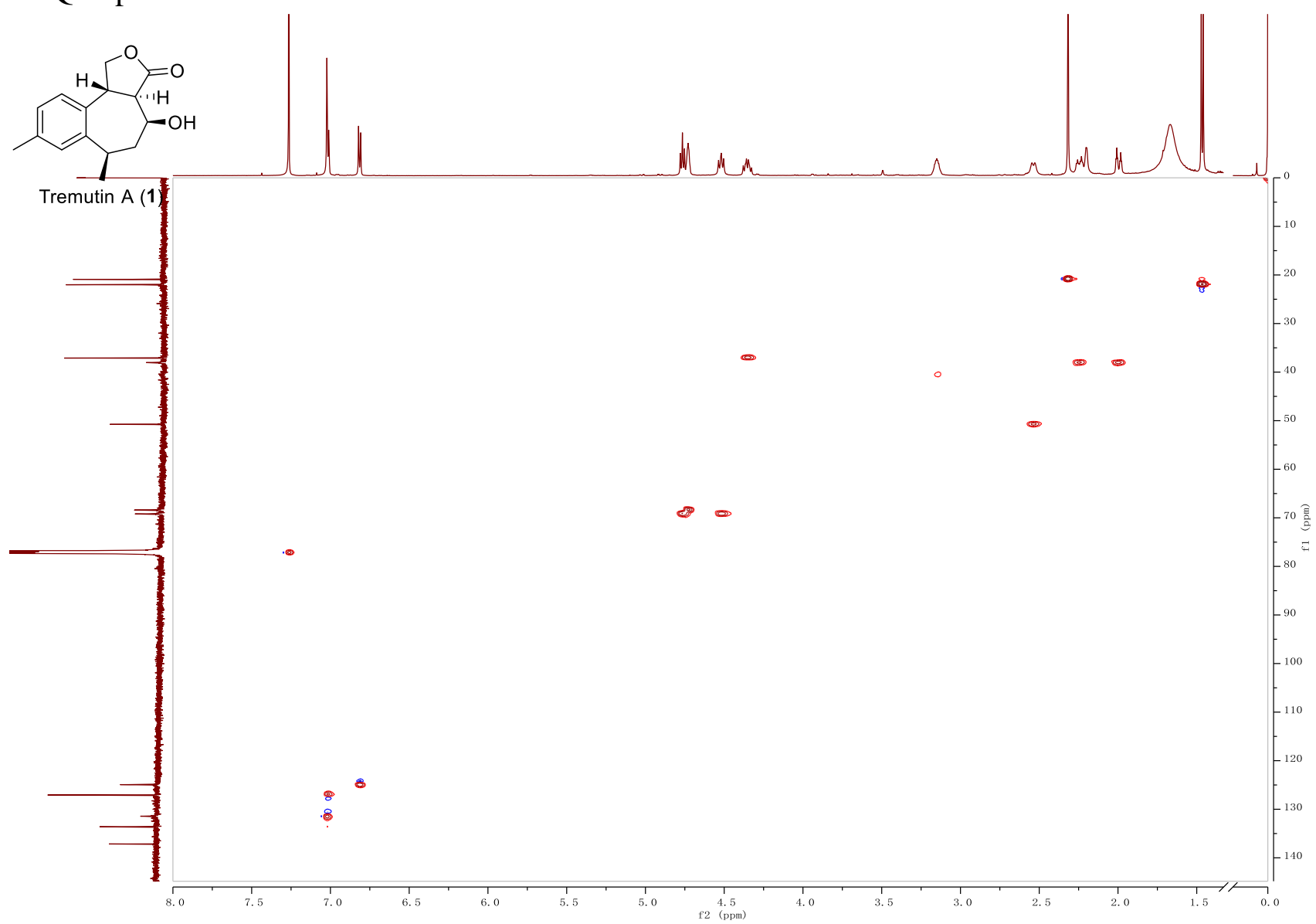

HMBC spectrum of $\mathbf{1}$

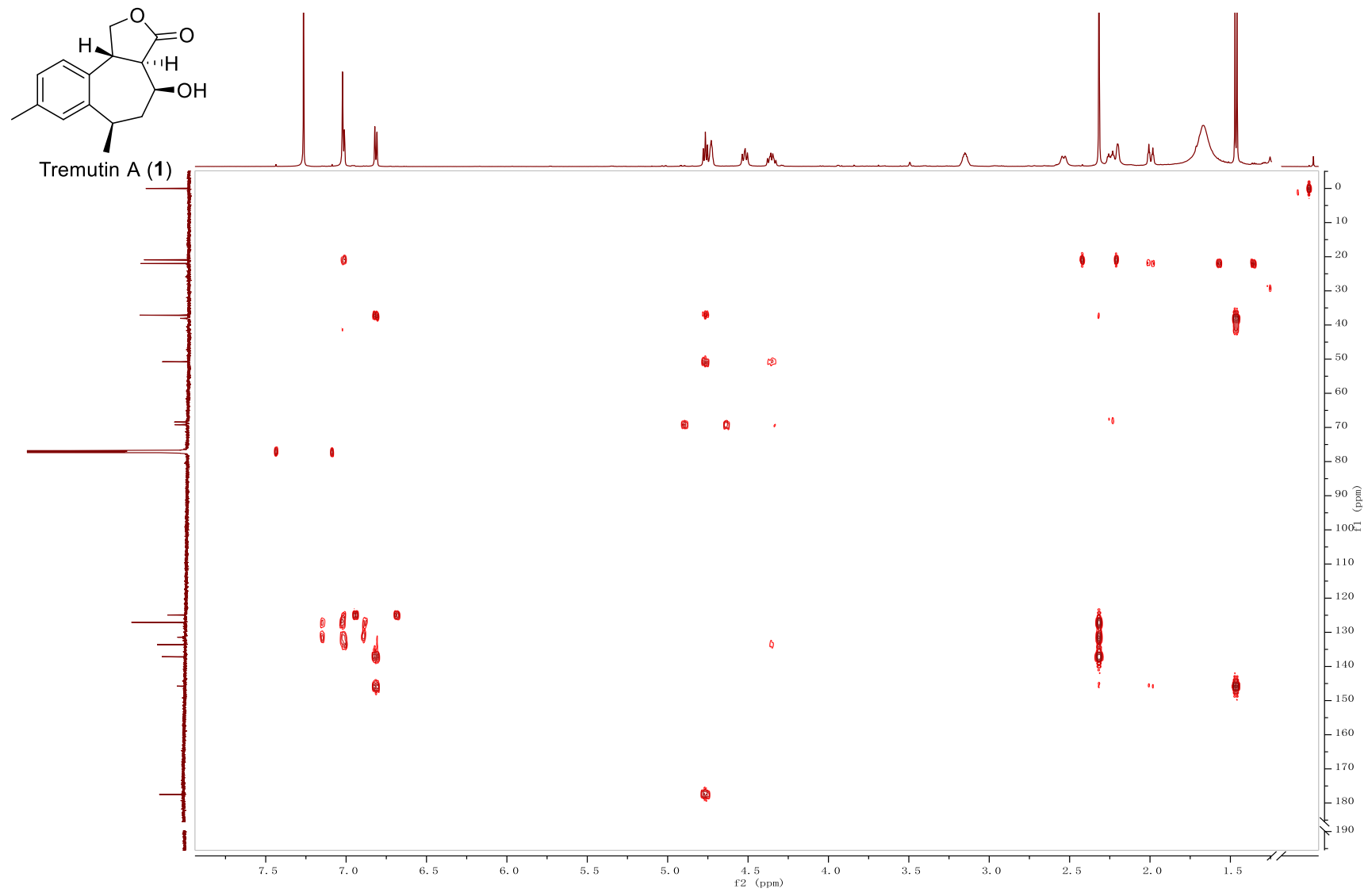


${ }^{1} \mathrm{H}-{ }^{1} \mathrm{H}$ COSY spectrum of $\mathbf{1}$

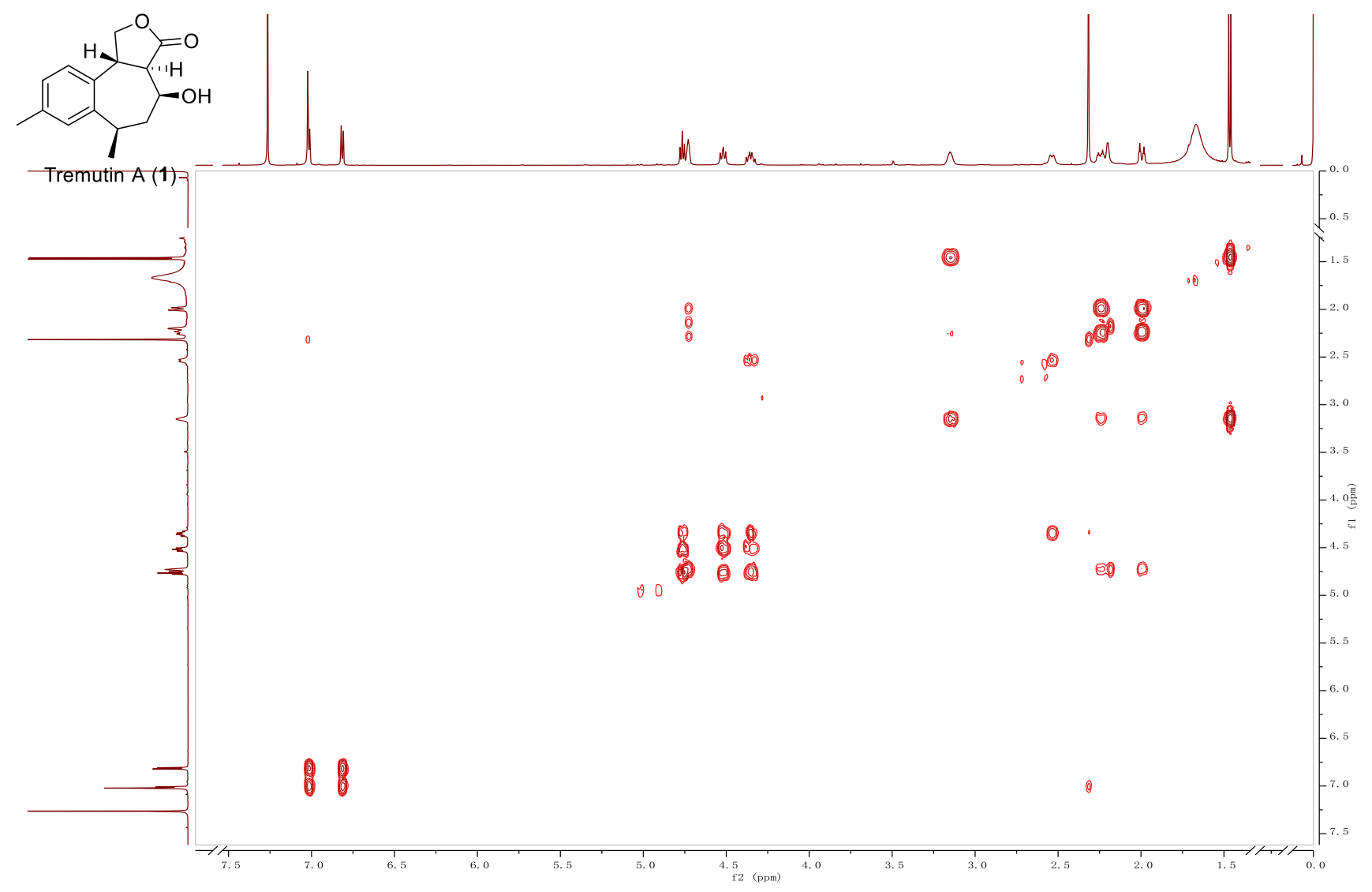

ROESY spectrum of $\mathbf{1}$

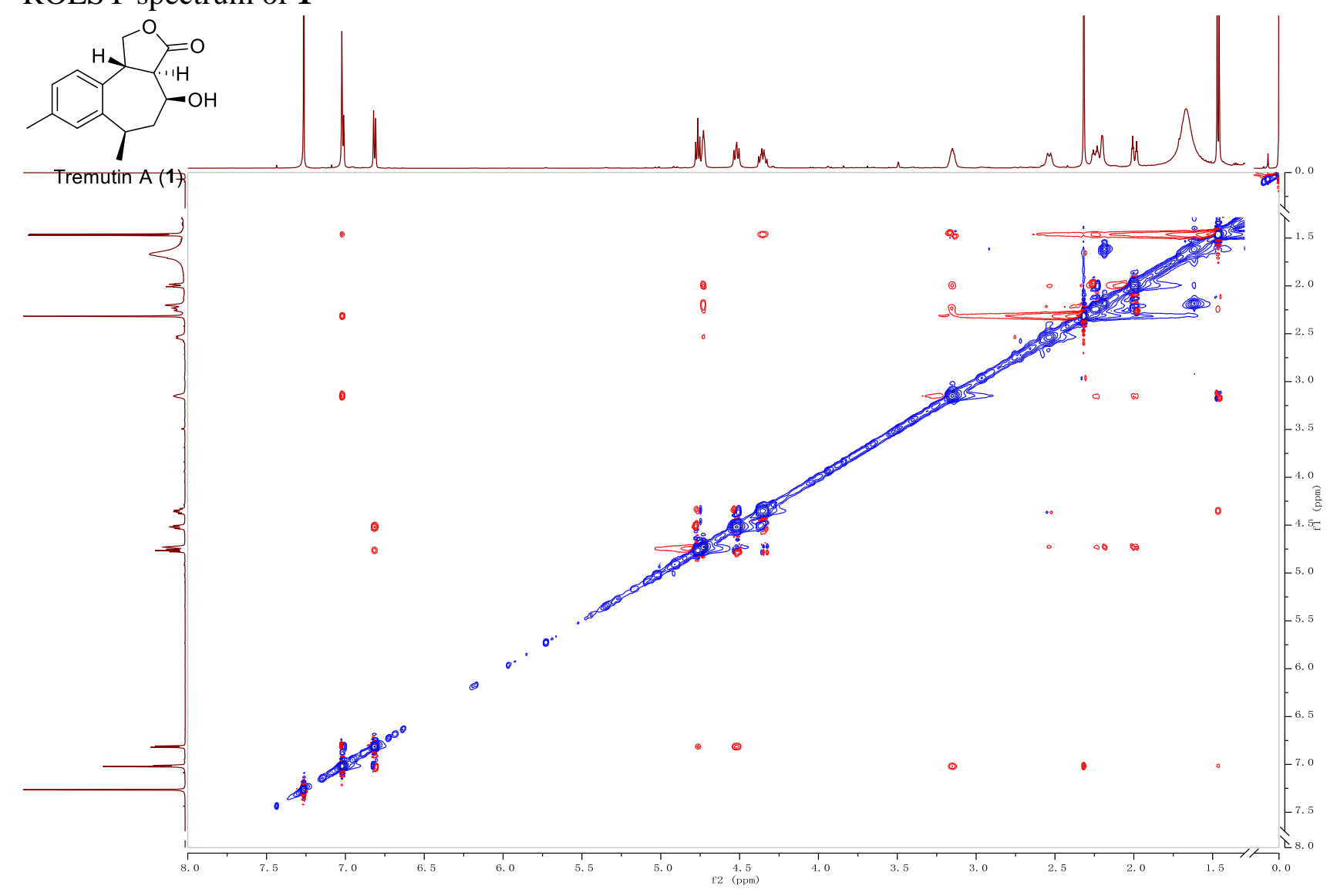




\section{HRESIMS of $\mathbf{1}$}

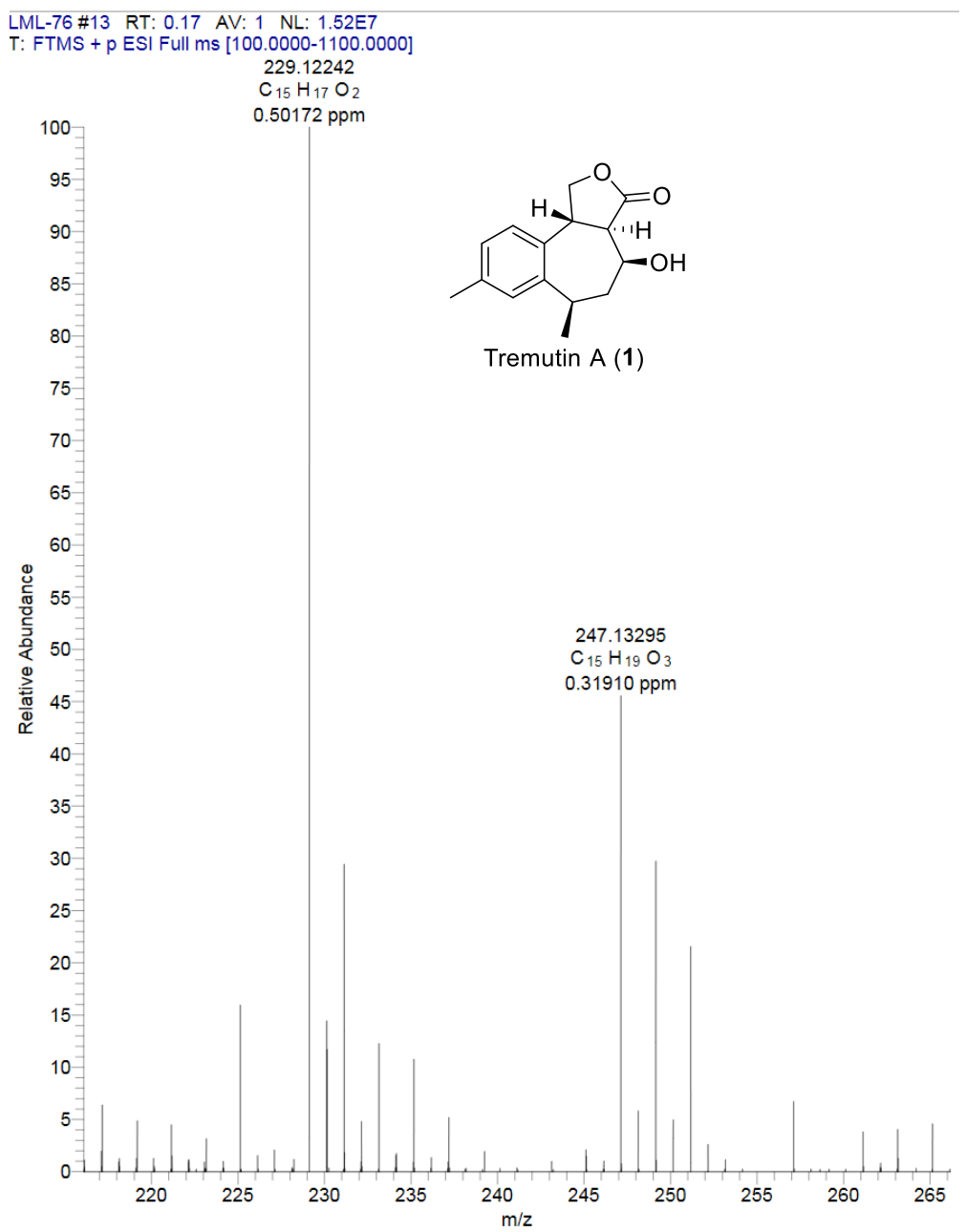

\section{CD spectrum of $\mathbf{1}$}

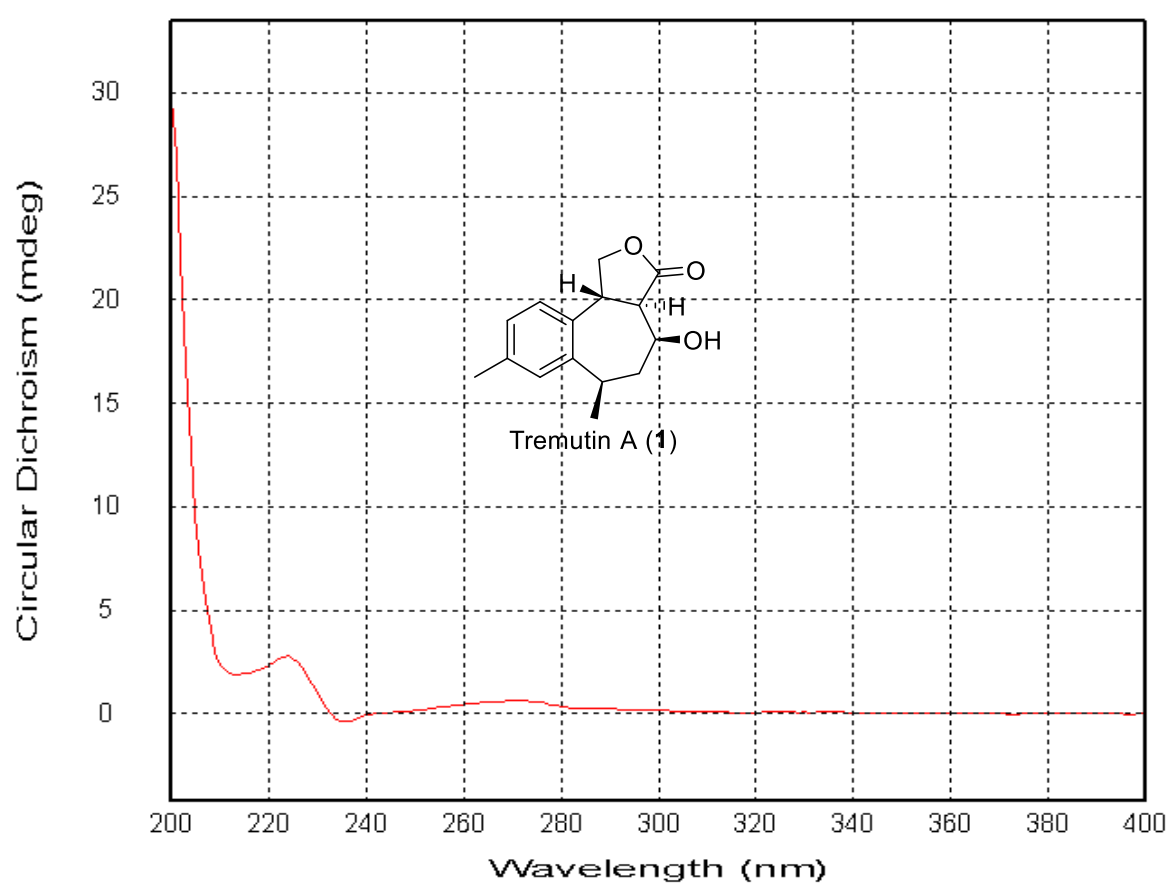

Smooth (s):0 
4.2 NMR, HRESIMS, and CD spectra for tremutin B (2)

${ }^{1} \mathrm{H}$ NMR spectrum of 2

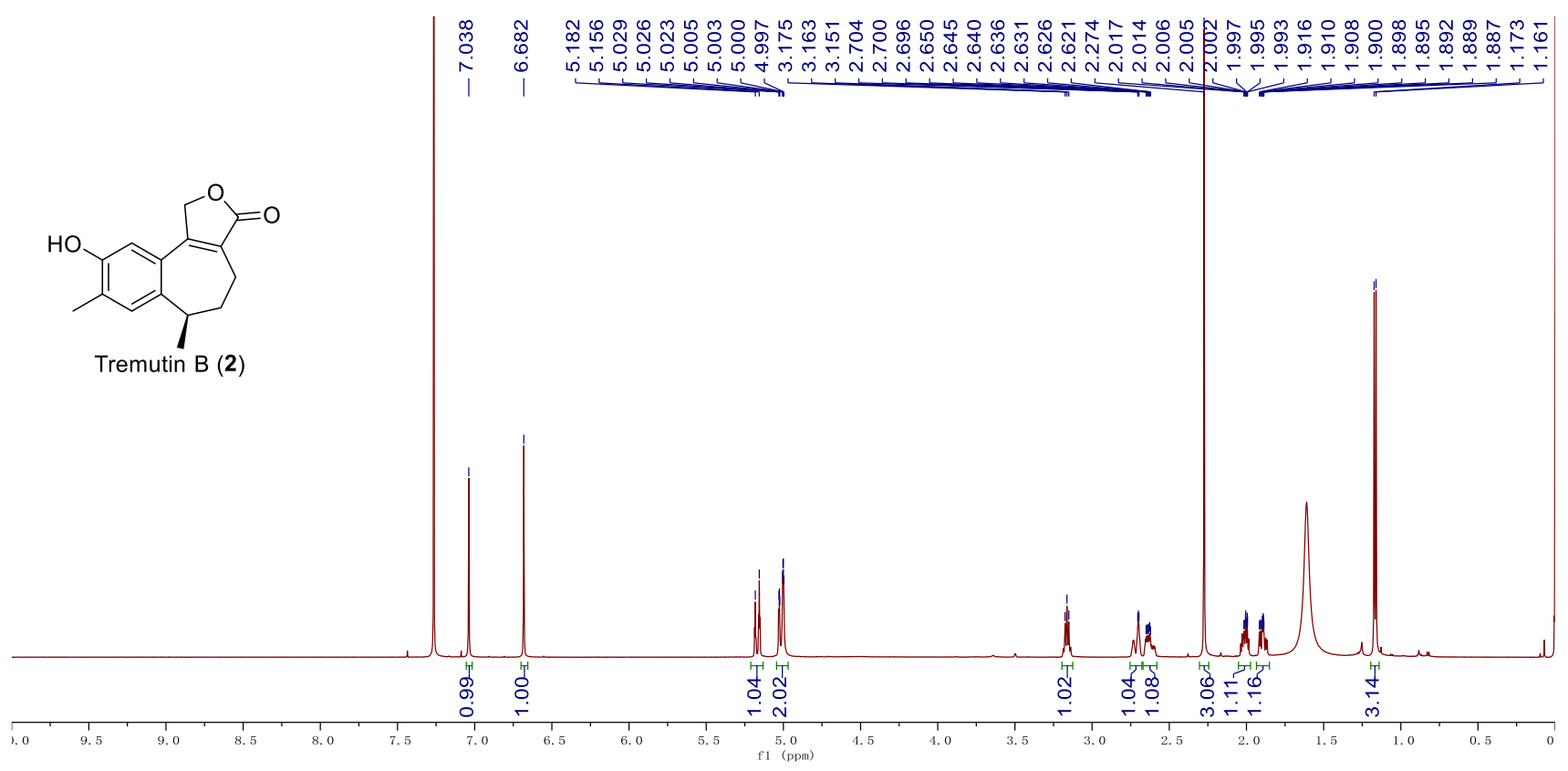

${ }^{13} \mathrm{C}$ NMR and DEPT spectra of 2

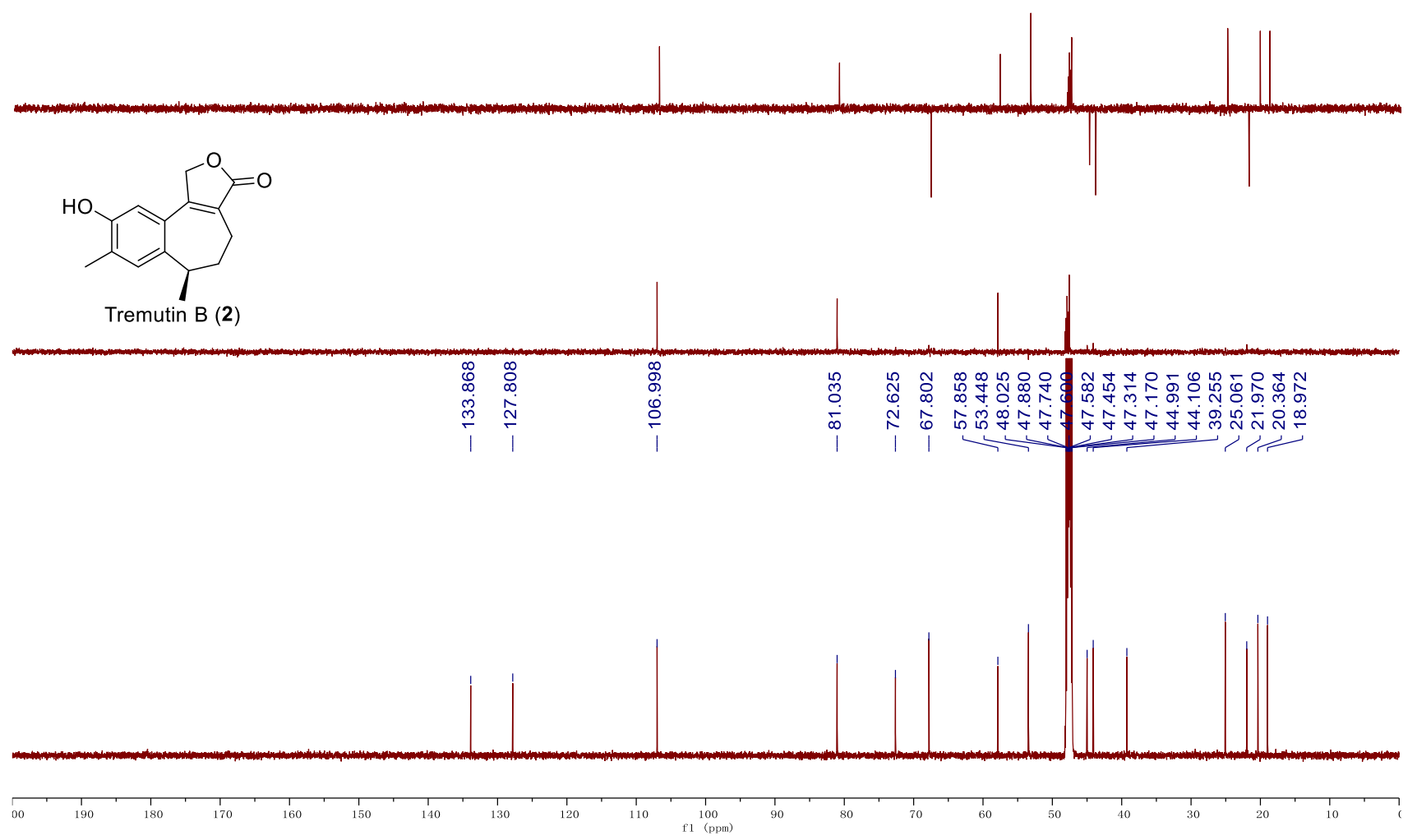


HSQC spectrum of $\mathbf{2}$

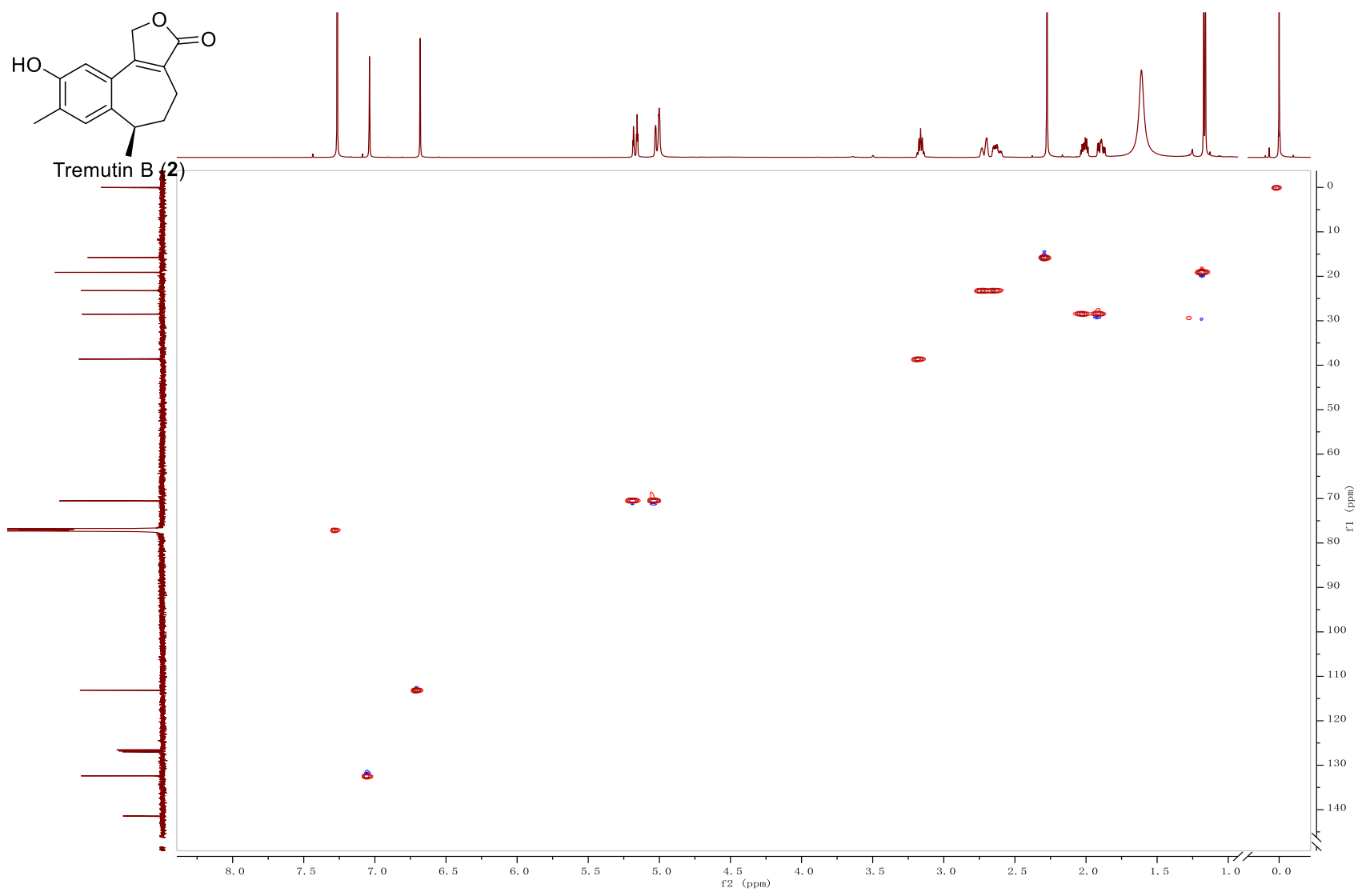

HMBC spectrum of $\mathbf{2}$

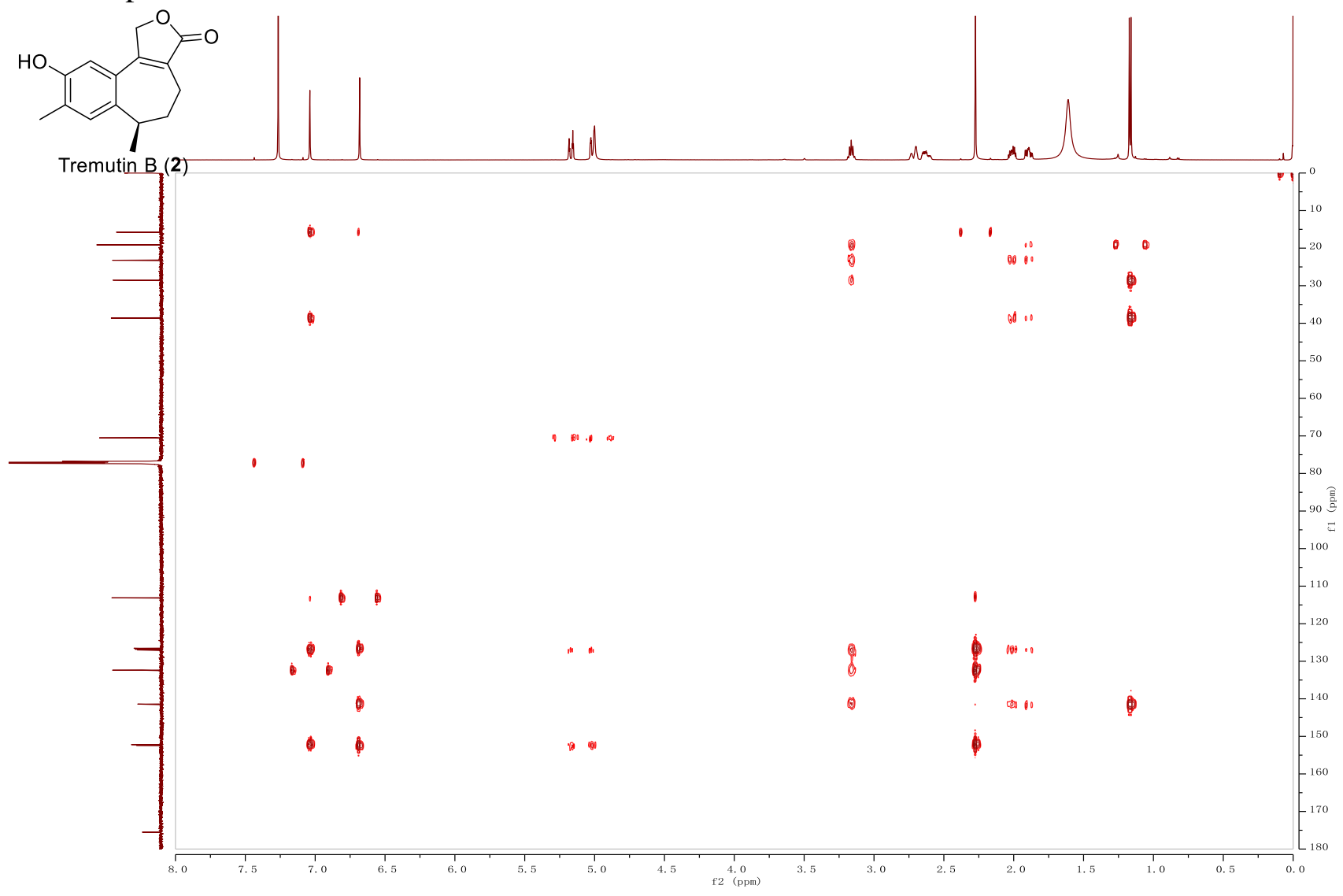




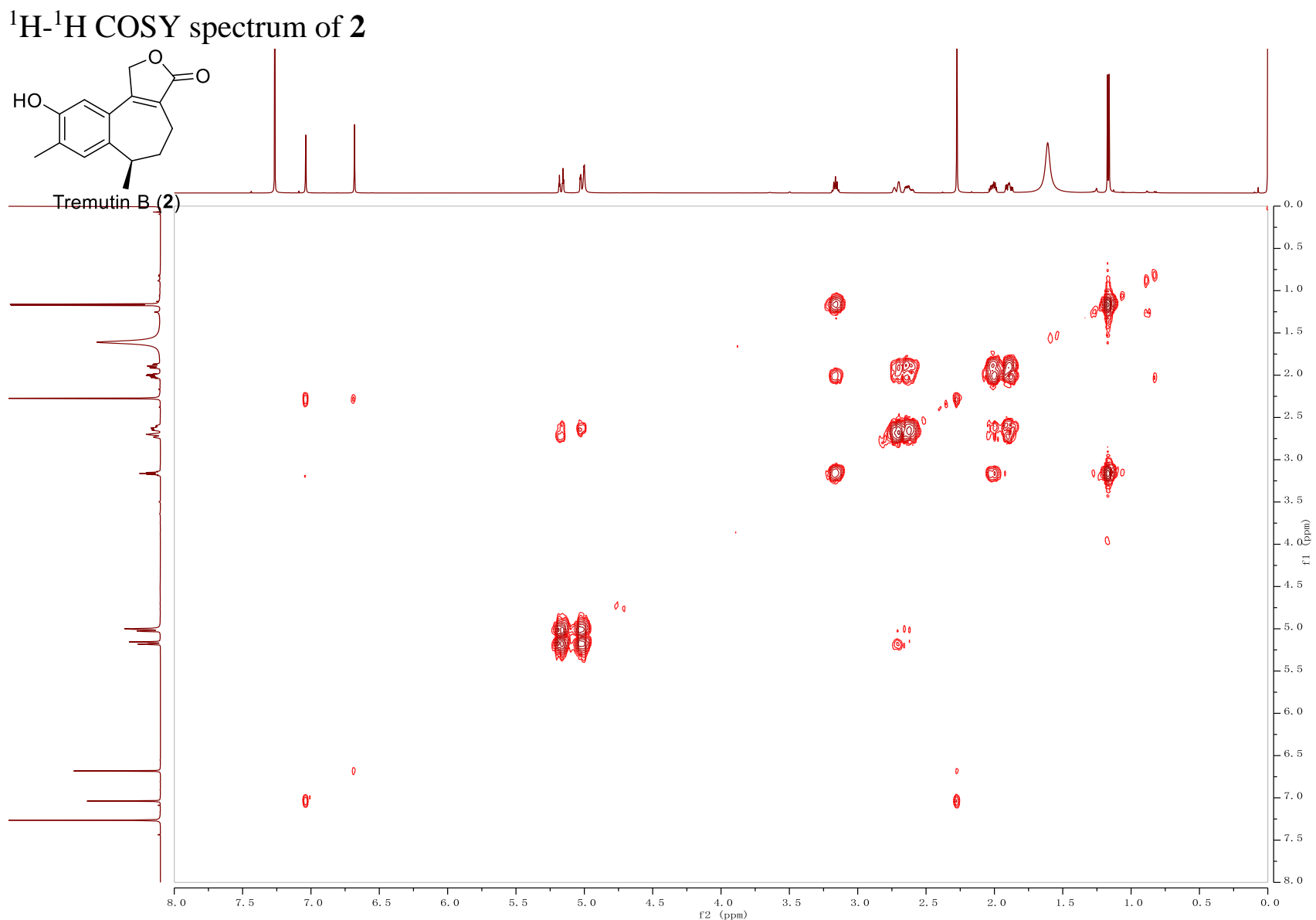

ROESY spectrum of $\mathbf{2}$

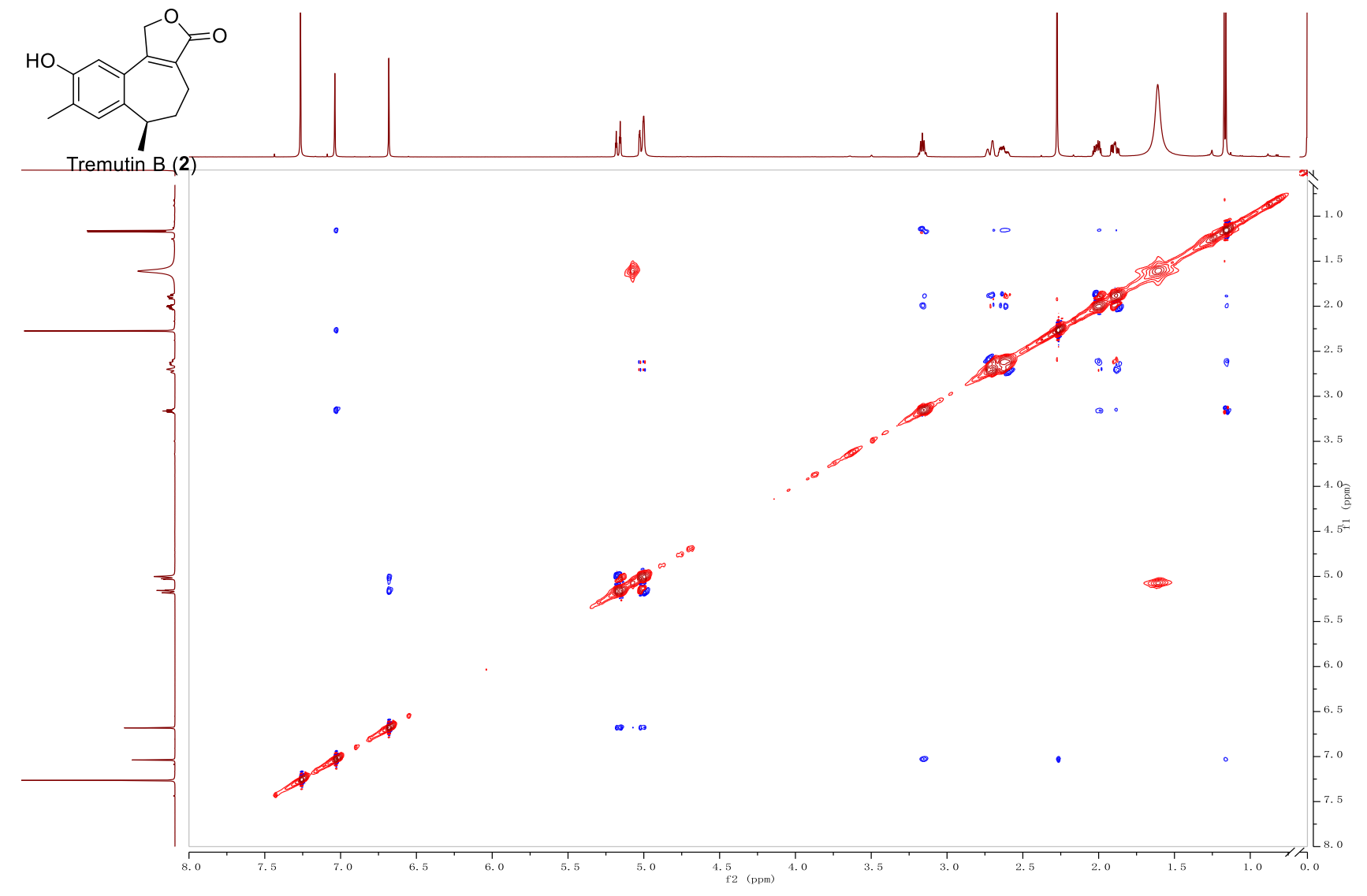




\section{HRESIMS of 2}

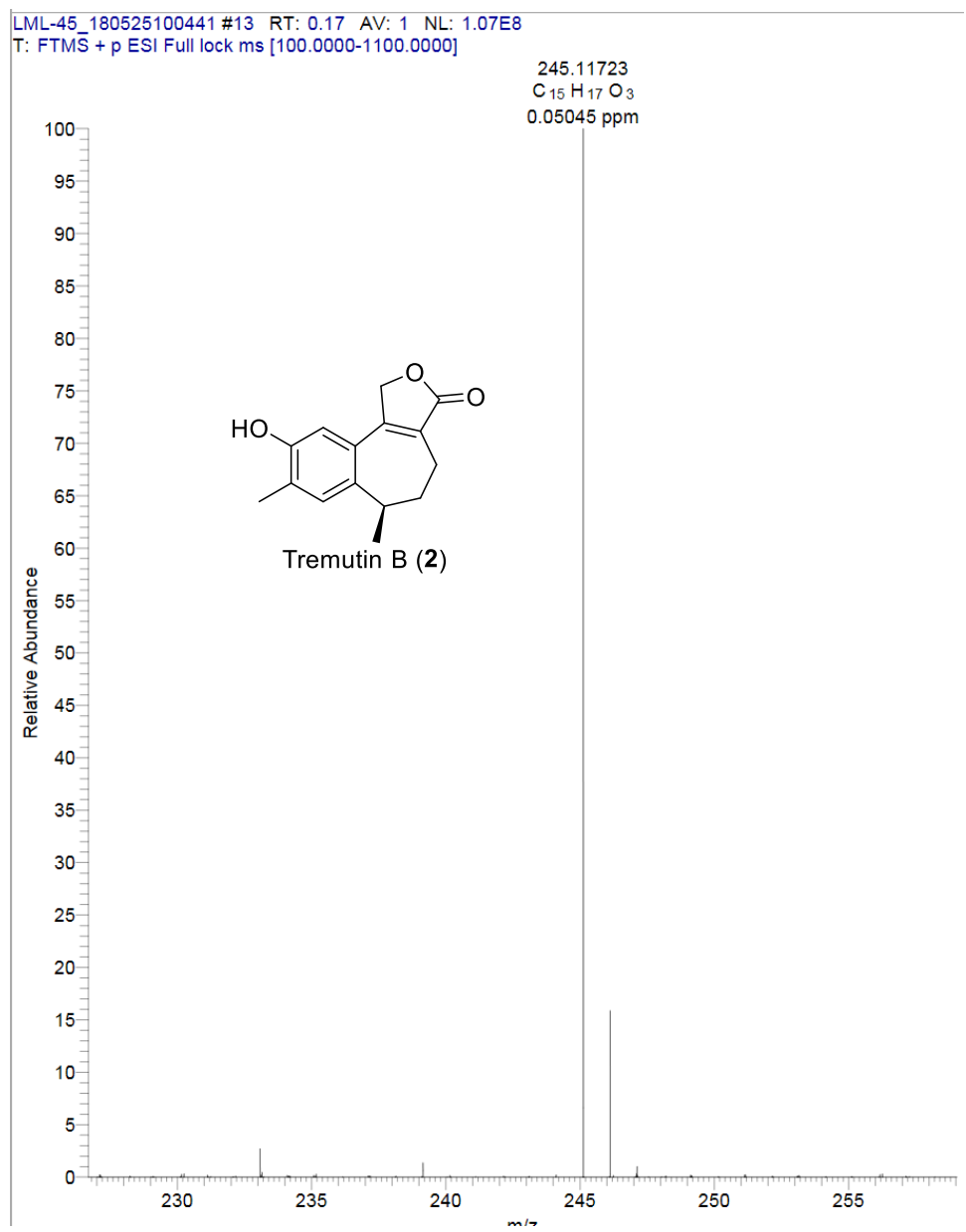

\section{CD spectrum of 2}

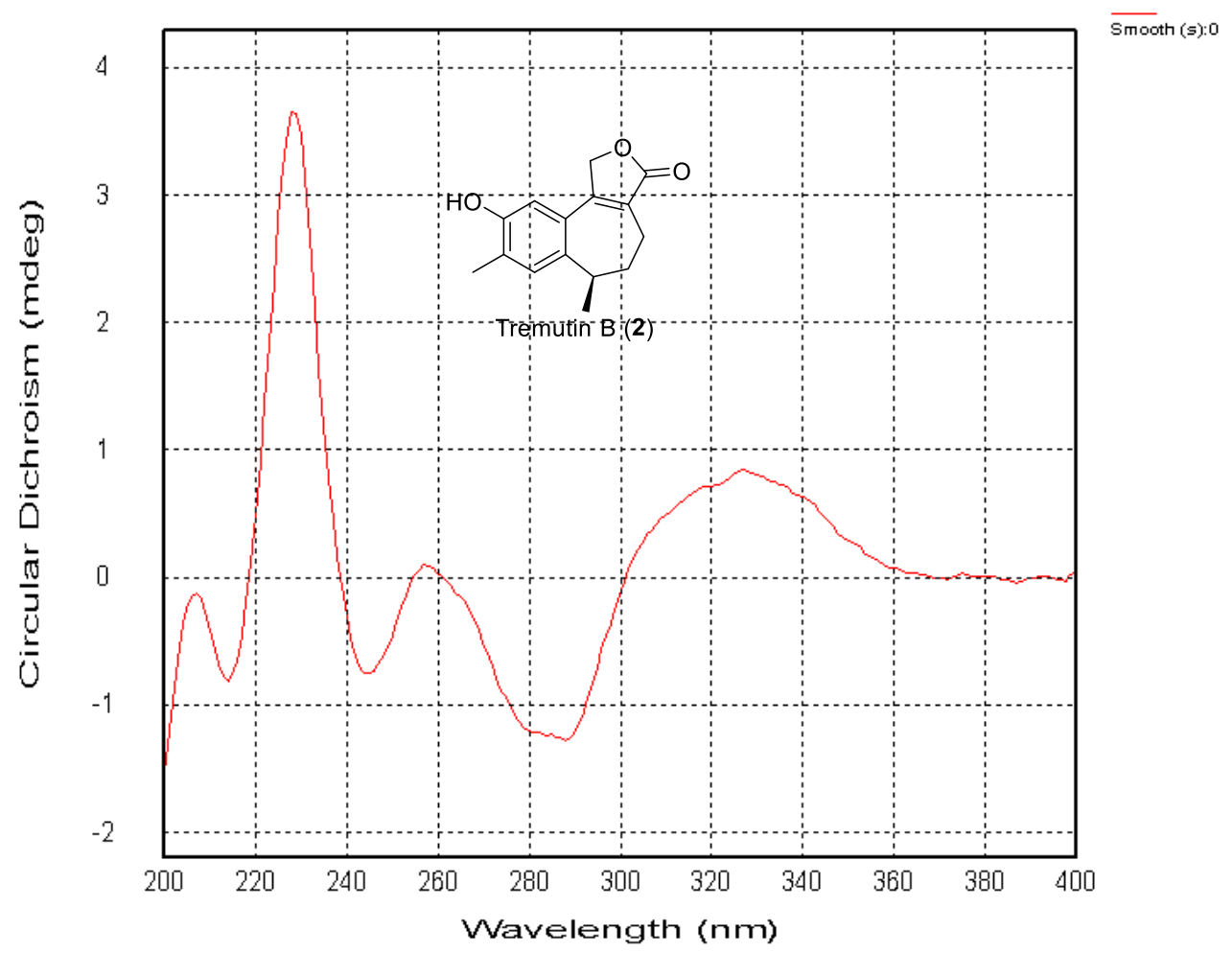


4.3 NMR and HRESIMS for tremutin C (3)

${ }^{1} \mathrm{H}$ NMR spectrum of $\mathbf{3}$

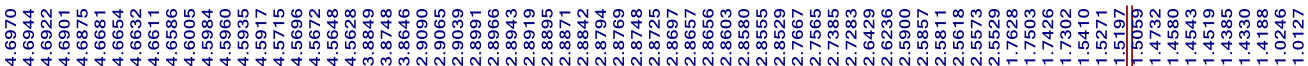

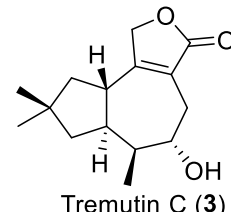

Tremutin C (3)

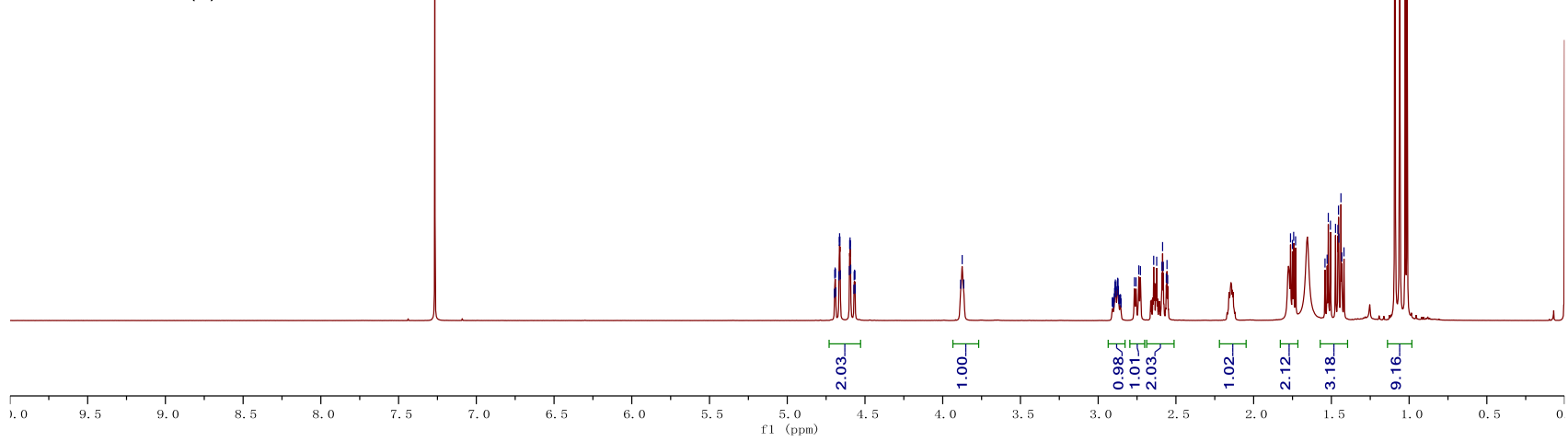

${ }^{13} \mathrm{C}$ NMR and DEPT spectra of 3
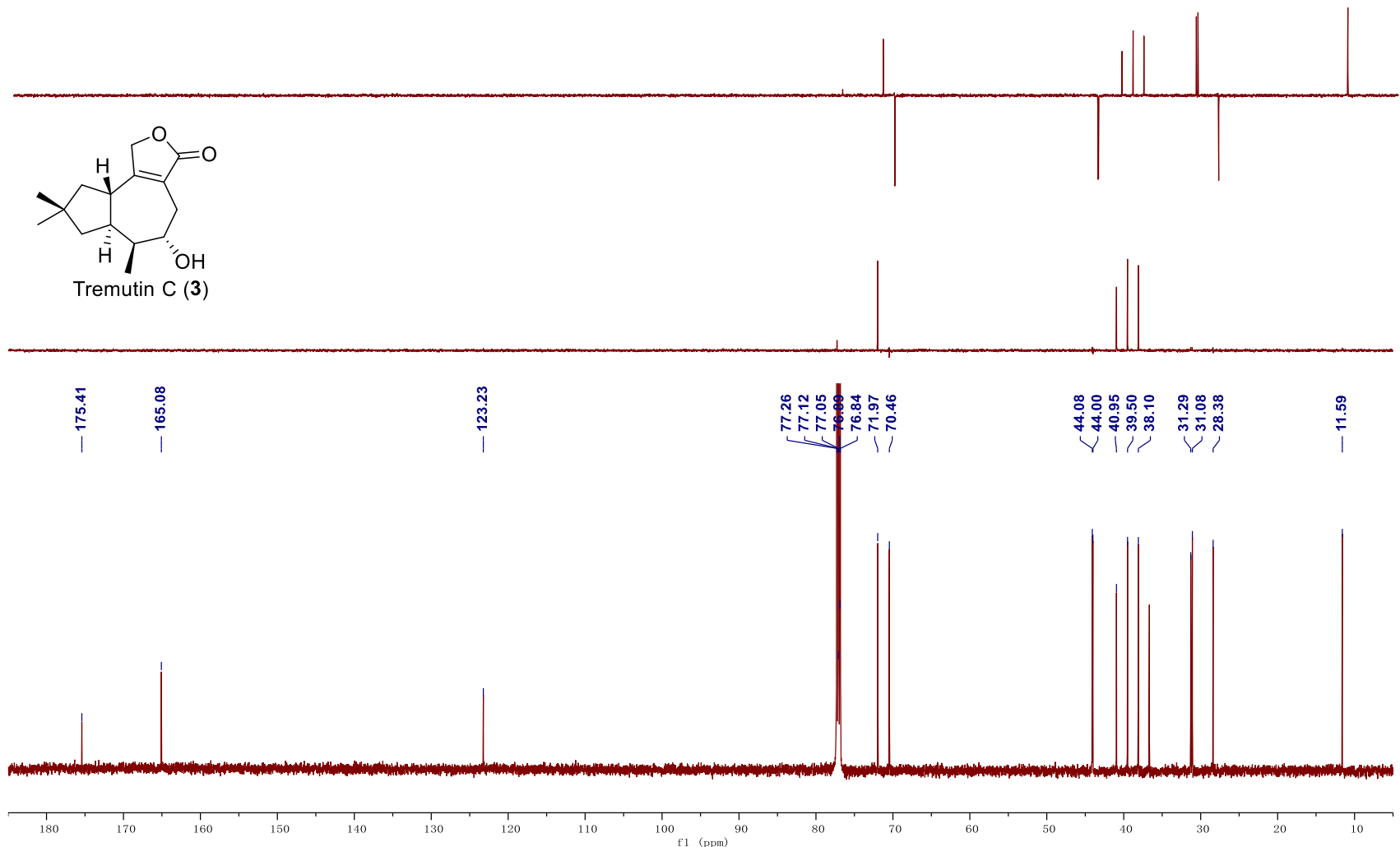
HSQC spectrum of $\mathbf{3}$

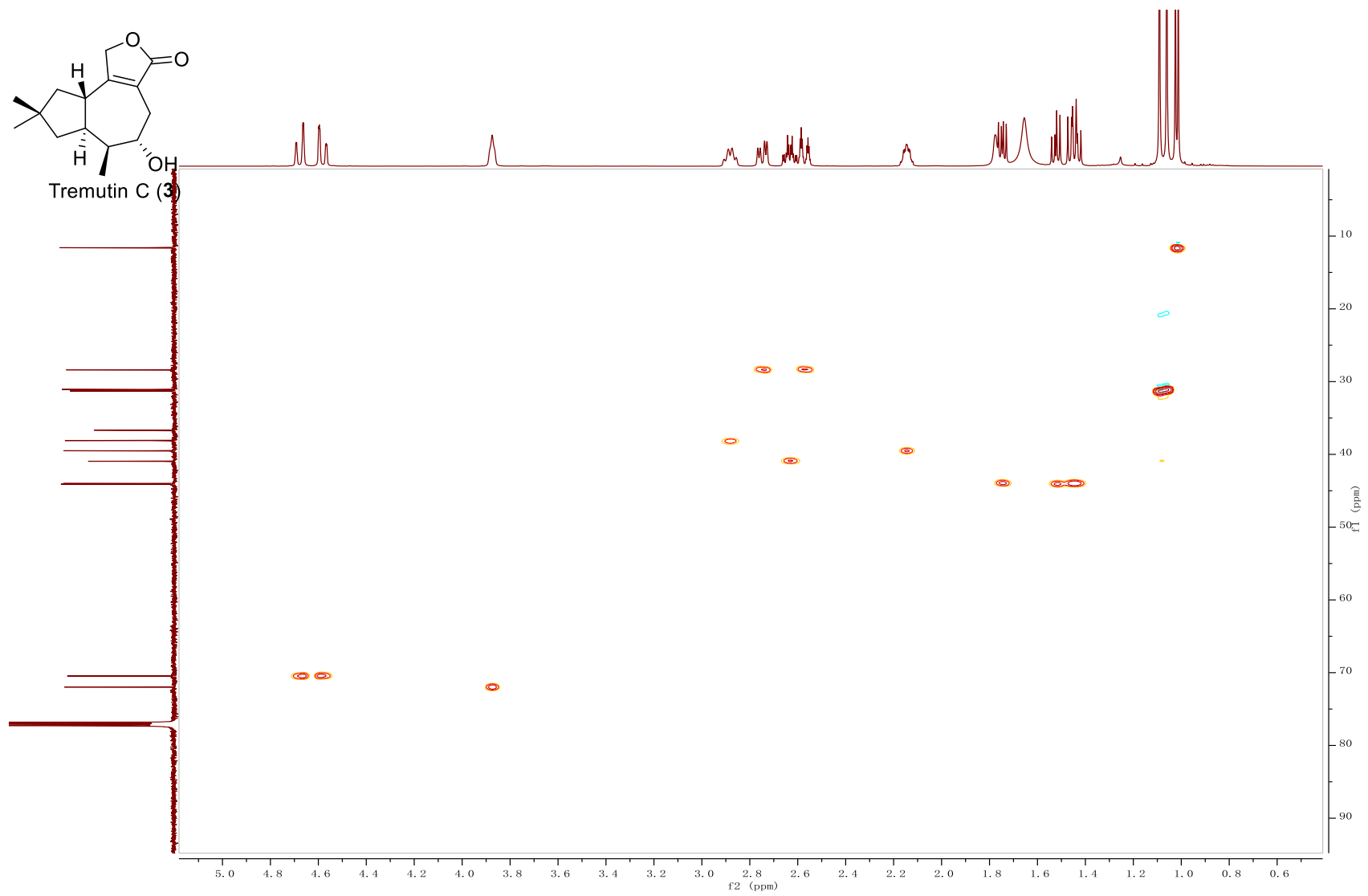

HMBC spectrum of $\mathbf{3}$

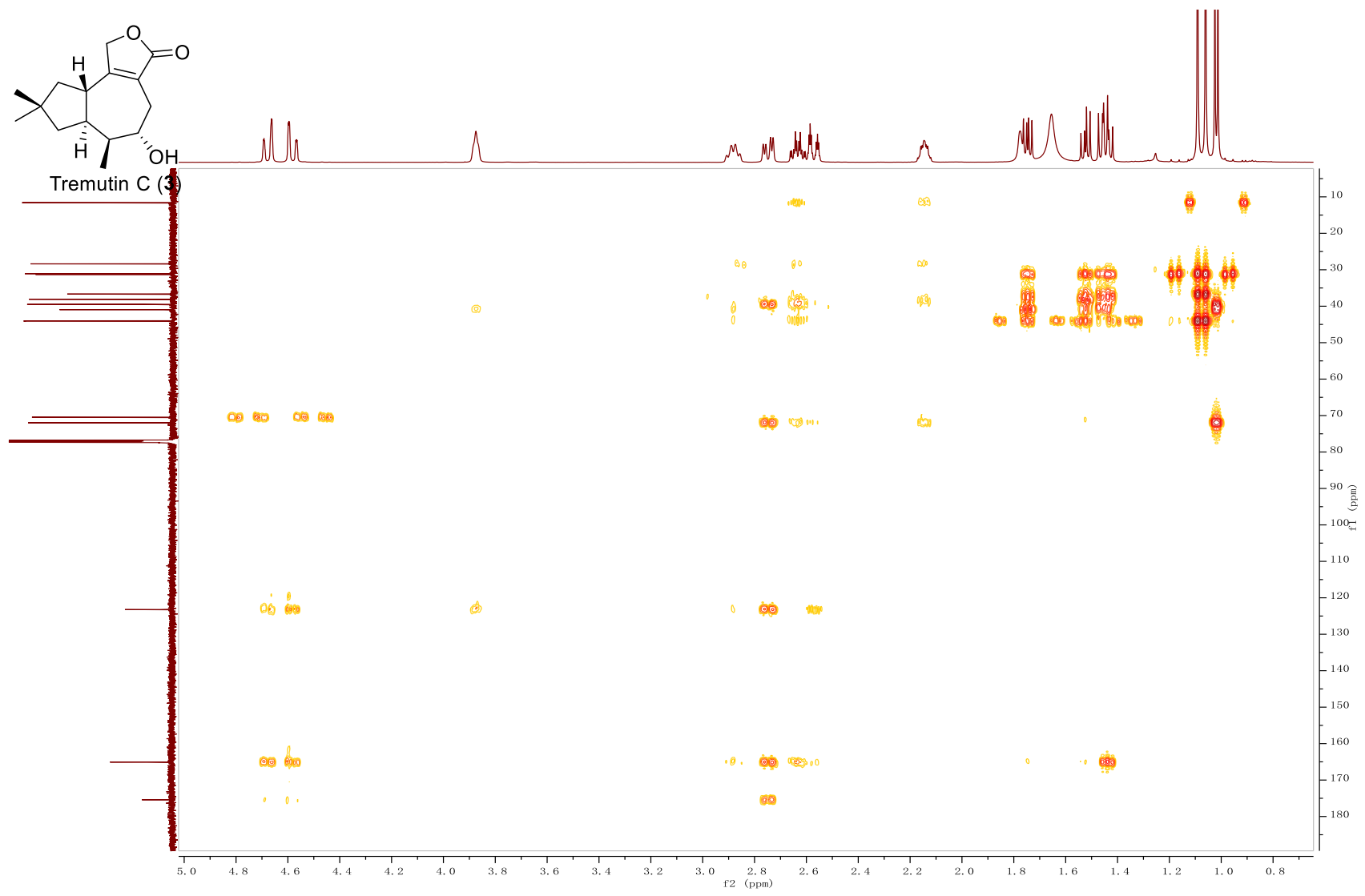


${ }^{1} \mathrm{H}-{ }^{1} \mathrm{H}$ COSY spectrum of $\mathbf{3}$

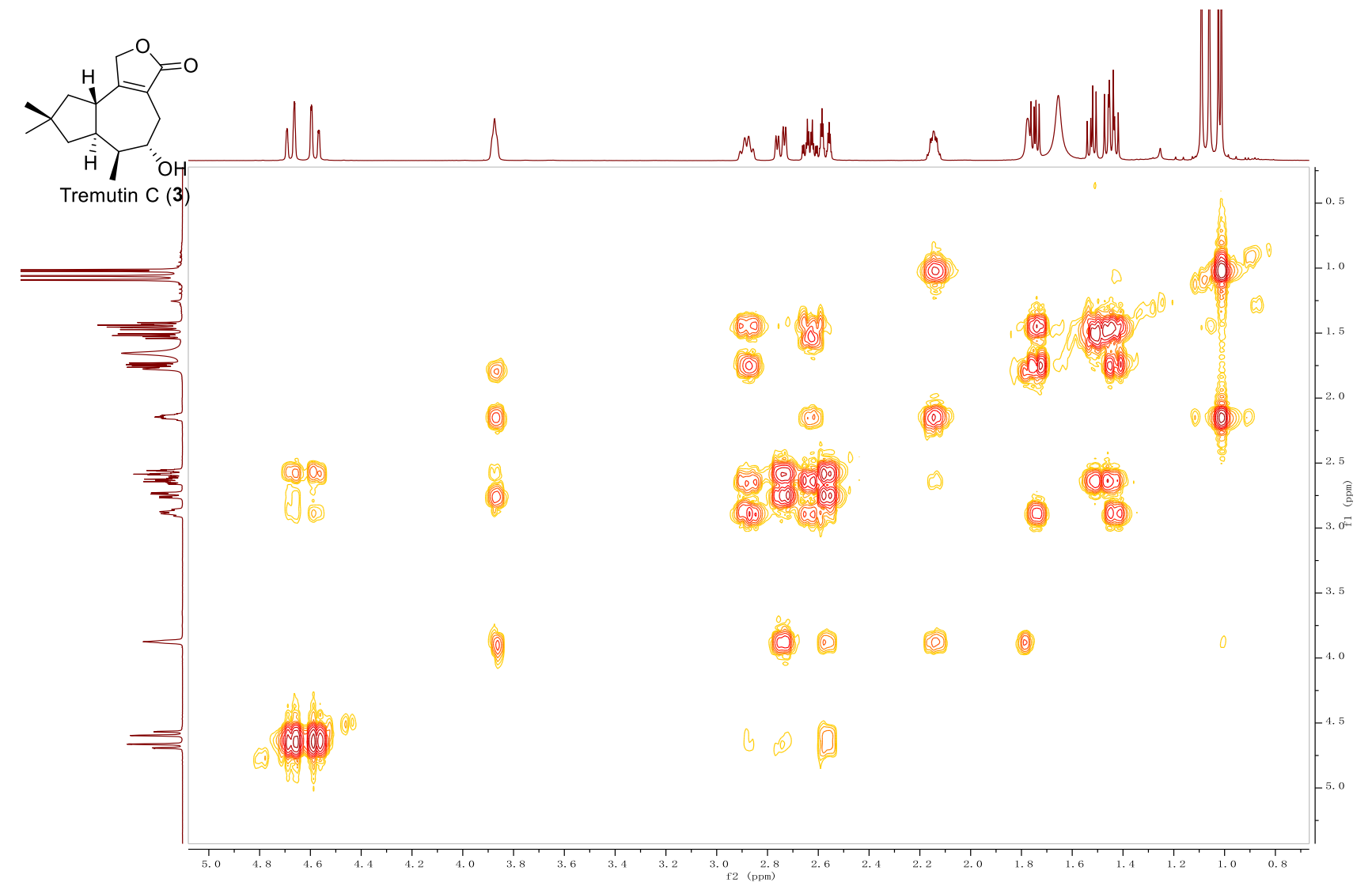

ROESY spectrum of $\mathbf{3}$

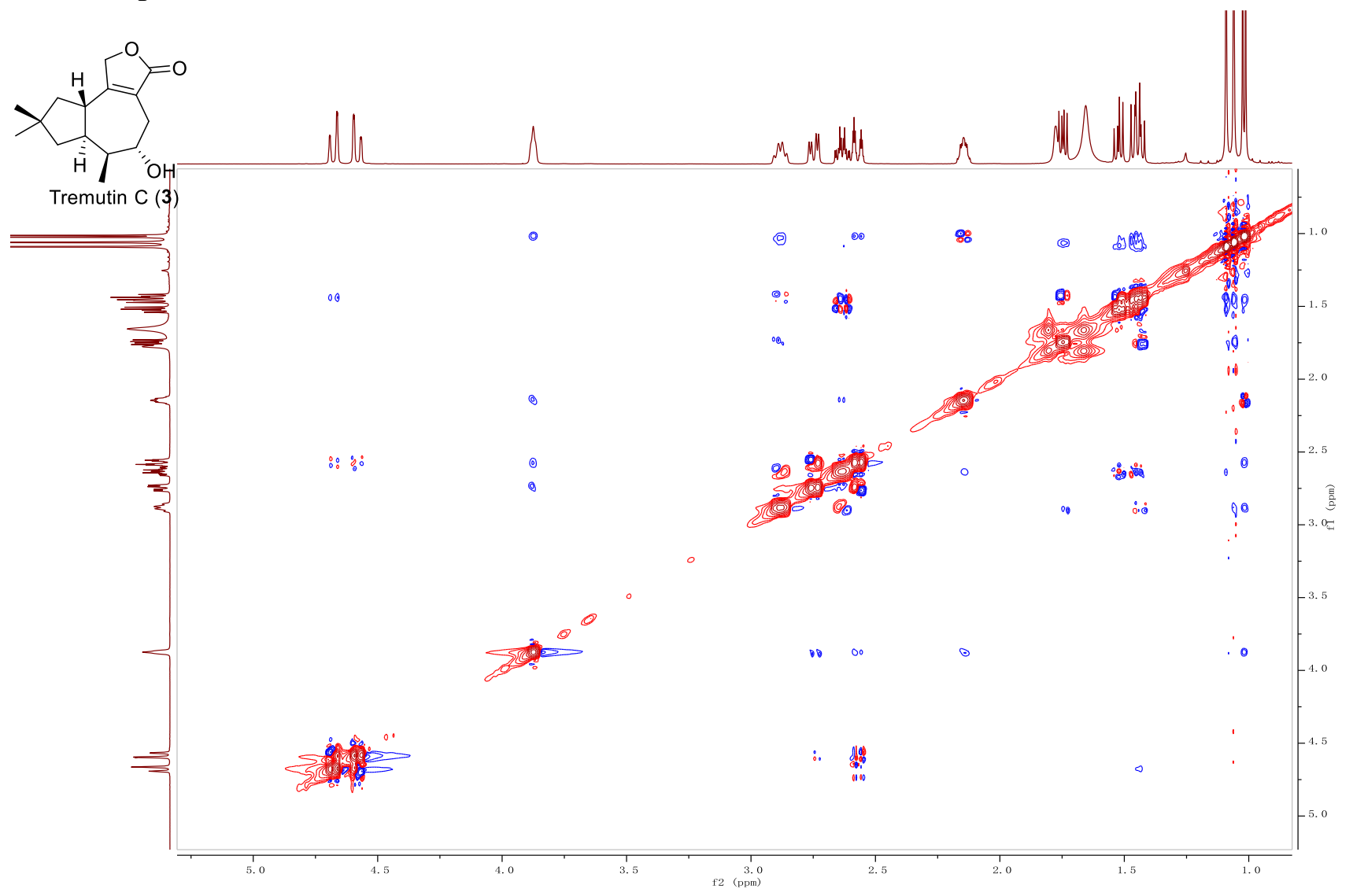




\section{HRESIMS of $\mathbf{3}$}

LML-74 \#13 RT: 0.17 AV: 1 NL: 2.57E8

T: FTMS + p ESI Full ms [100.0000-1100.0000]

251.16411

$\mathrm{C}_{15} \mathrm{H}_{23} \mathrm{O}_{3}$

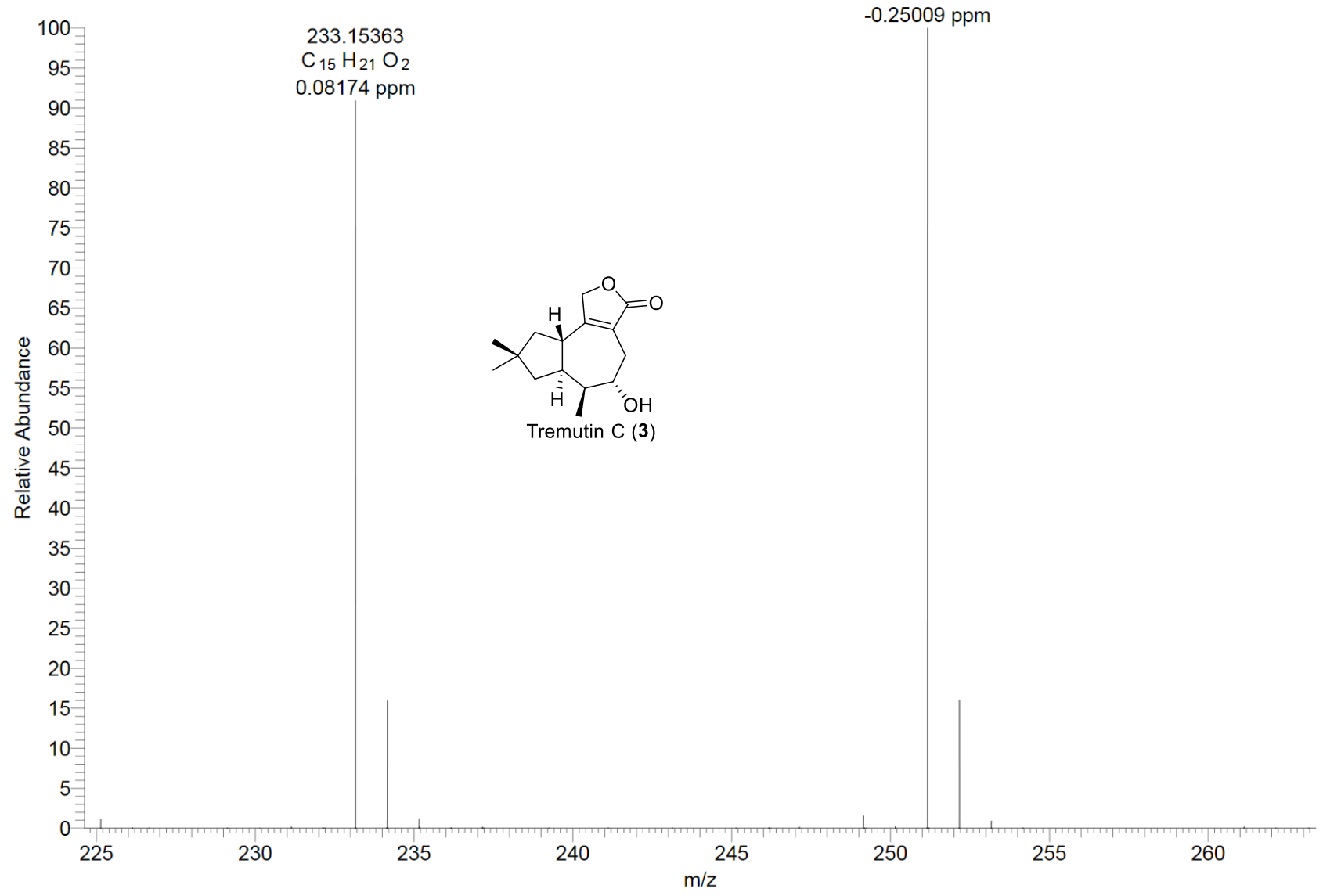


4.4 NMR and HRESIMS for tremutin D (4)

${ }^{1} \mathrm{H}$ NMR spectrum of 4

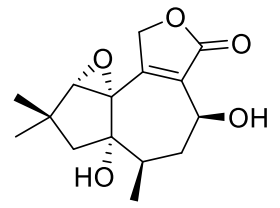

Tremutin D (4)

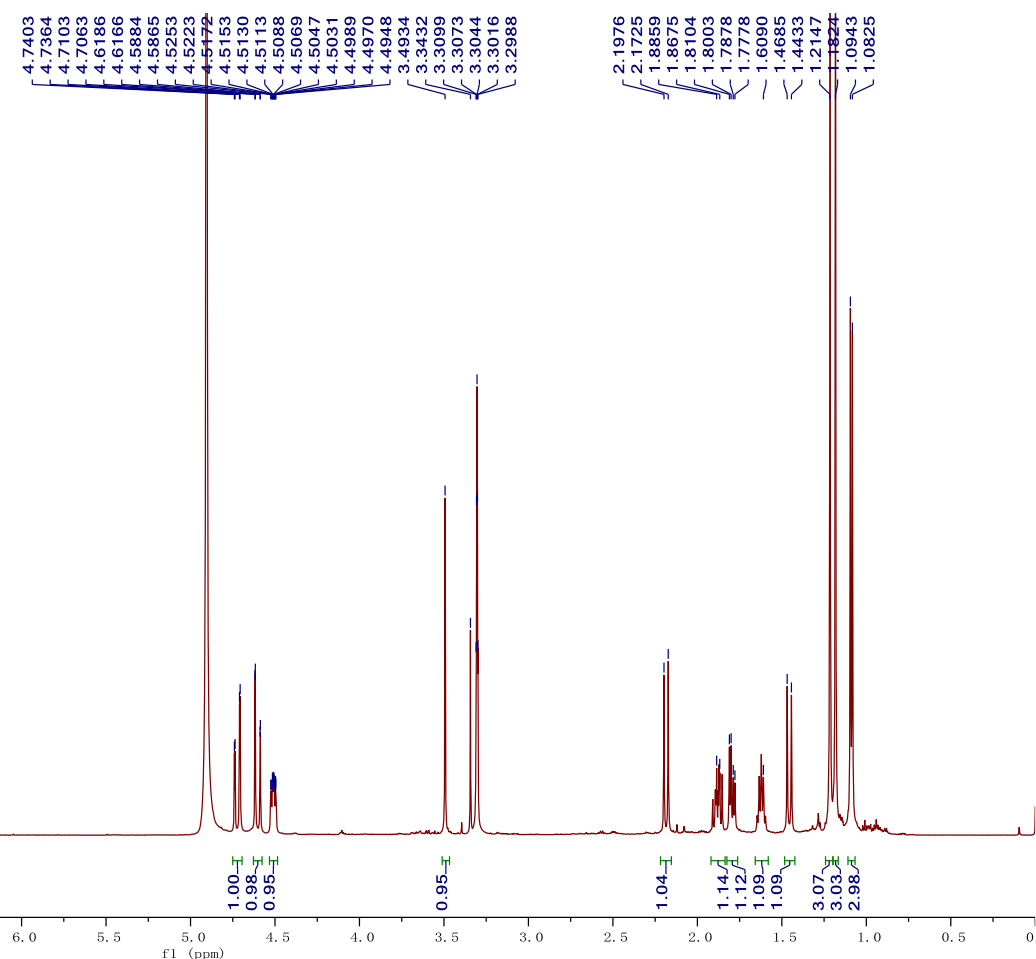

${ }^{13} \mathrm{C}$ NMR and DEPT spectra of 4
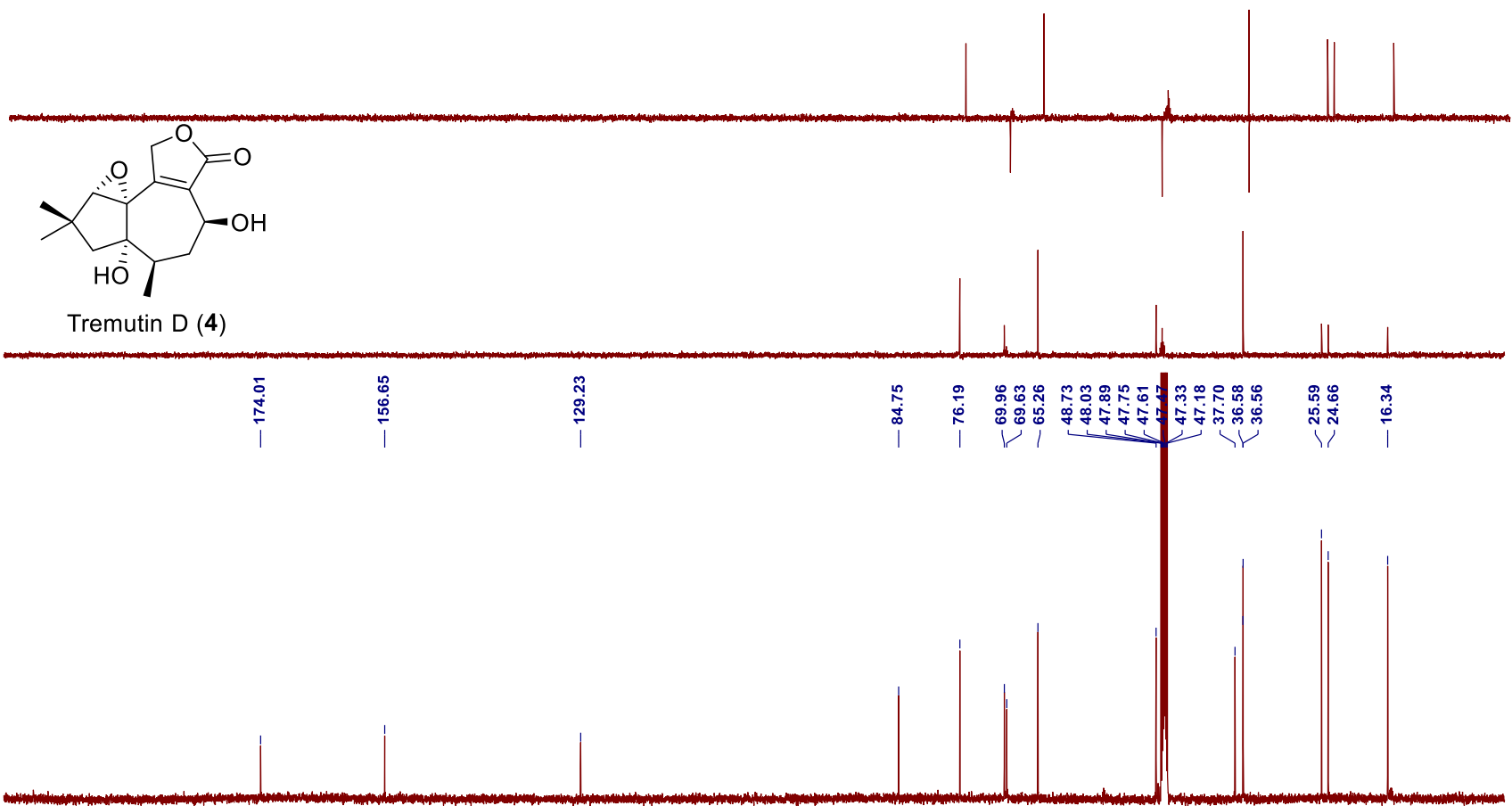

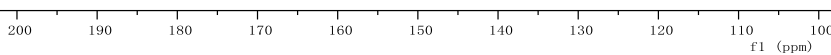




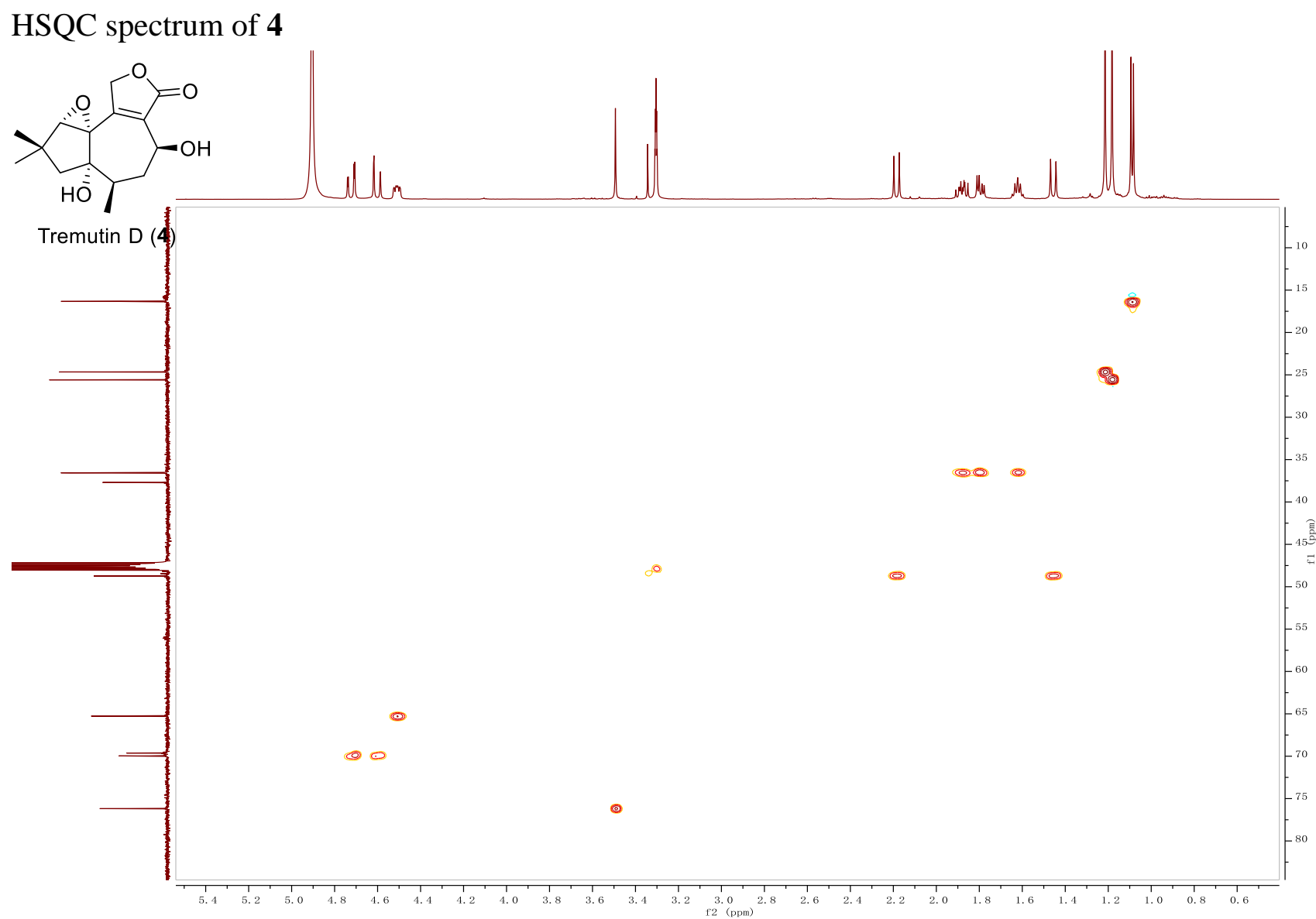

HMBC spectrum of $\mathbf{4}$

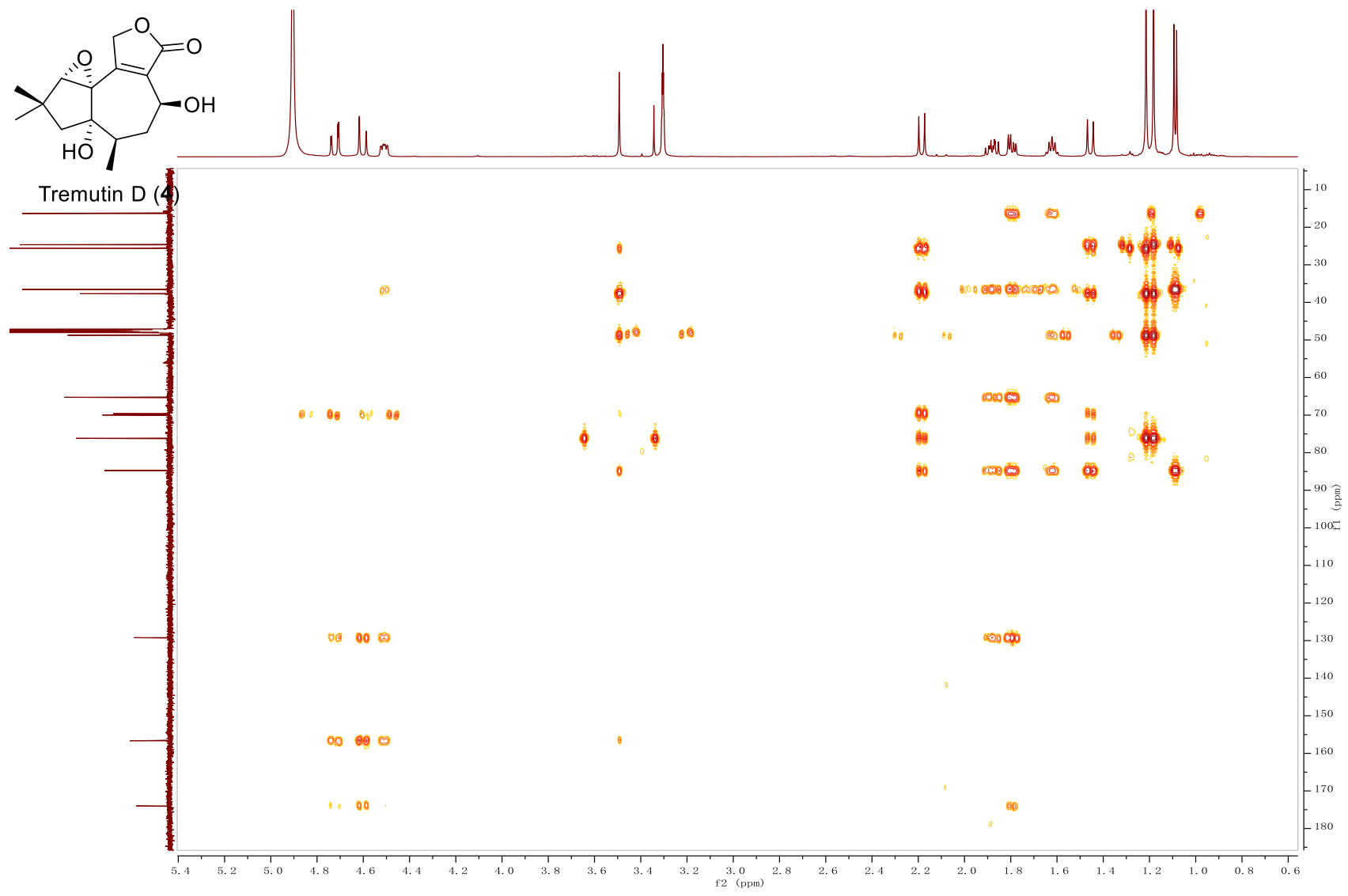




\section{${ }^{1} \mathrm{H}-{ }^{1} \mathrm{H}$ COSY spectrum of 4}

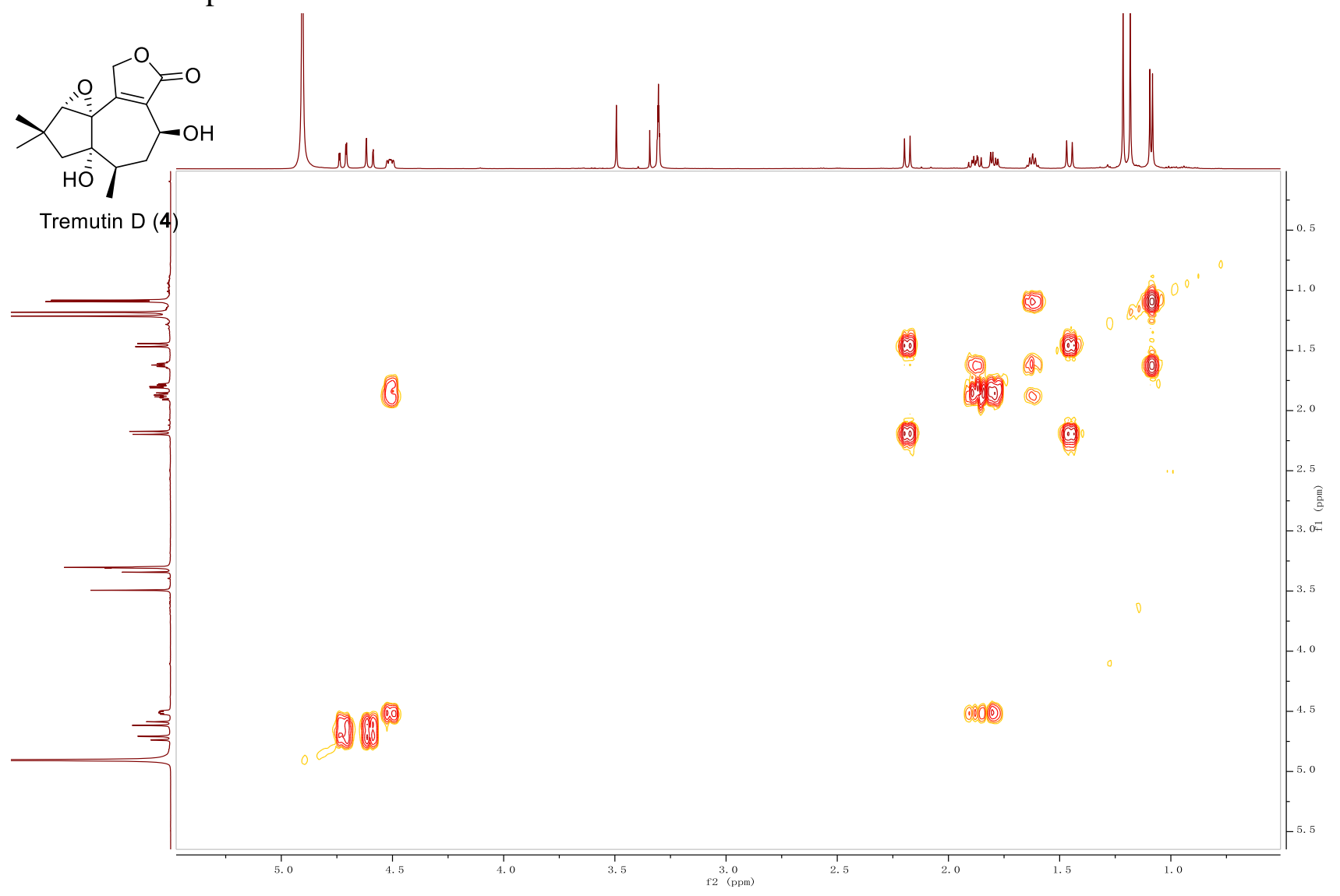

ROESY spectrum of $\mathbf{4}$

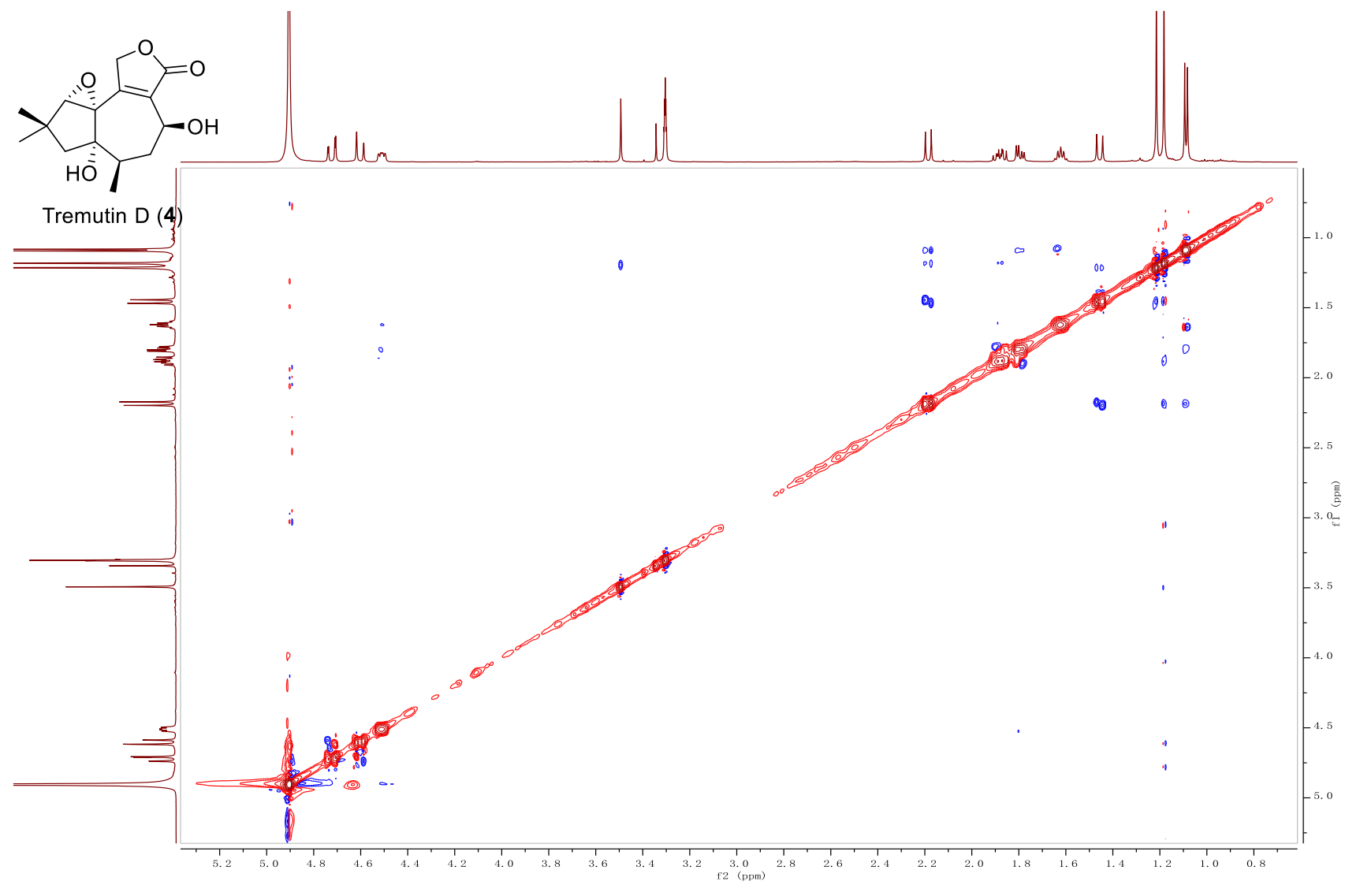


HRESIMS of 4

LML-182\#13 RT: 0.17 AV: 1 NL: 5.99E6

T: FTMS + p ESI Full lock ms [150.0000-1100.0000]

$$
\begin{gathered}
303.12024 \\
\mathrm{C}_{15} \mathrm{H}_{20} \mathrm{O}_{5} \mathrm{Na}
\end{gathered}
$$

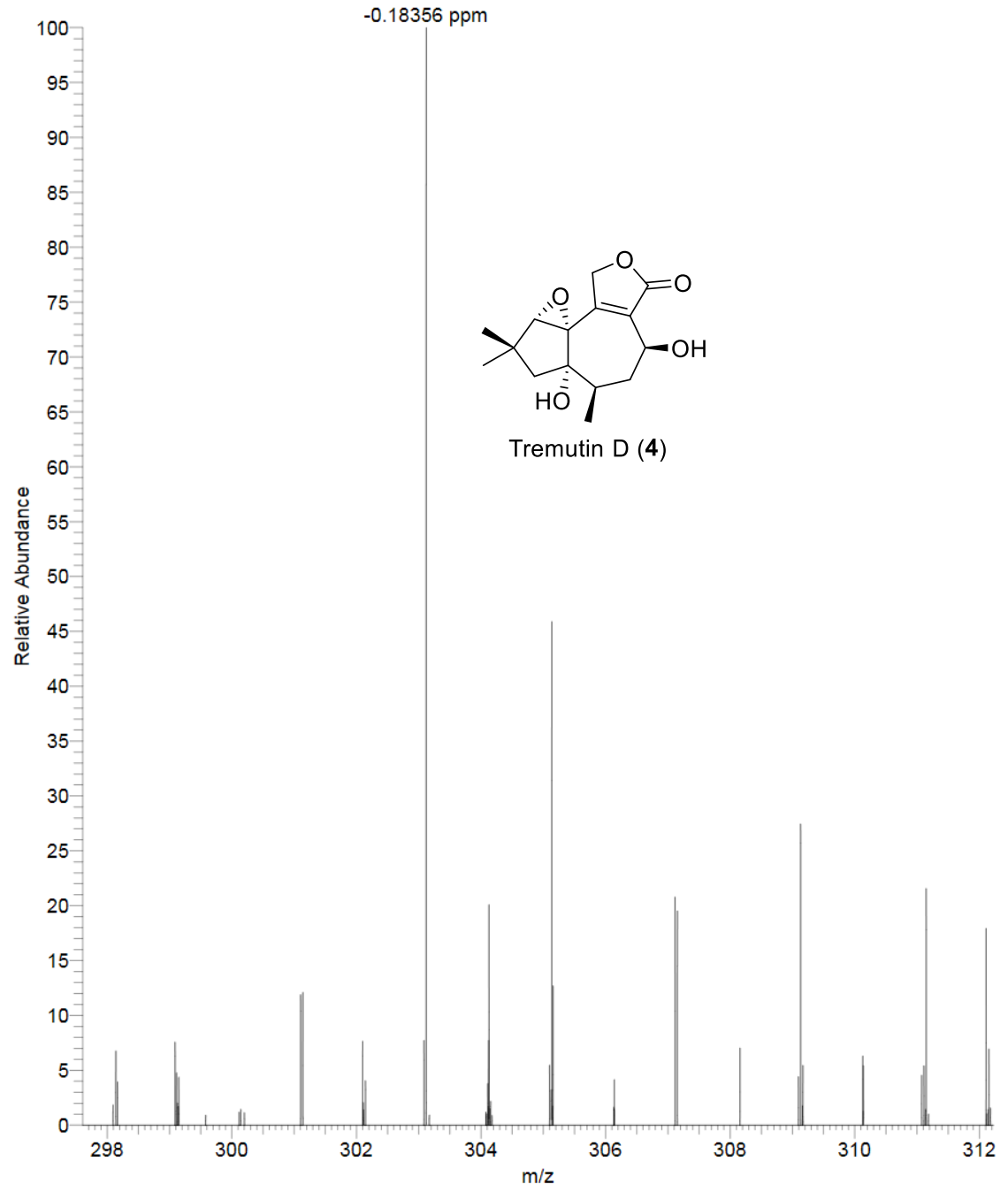


4.5 NMR and HRESIMS for tremutin E (5)

${ }^{1} \mathrm{H}$ NMR spectrum of $\mathbf{5}$

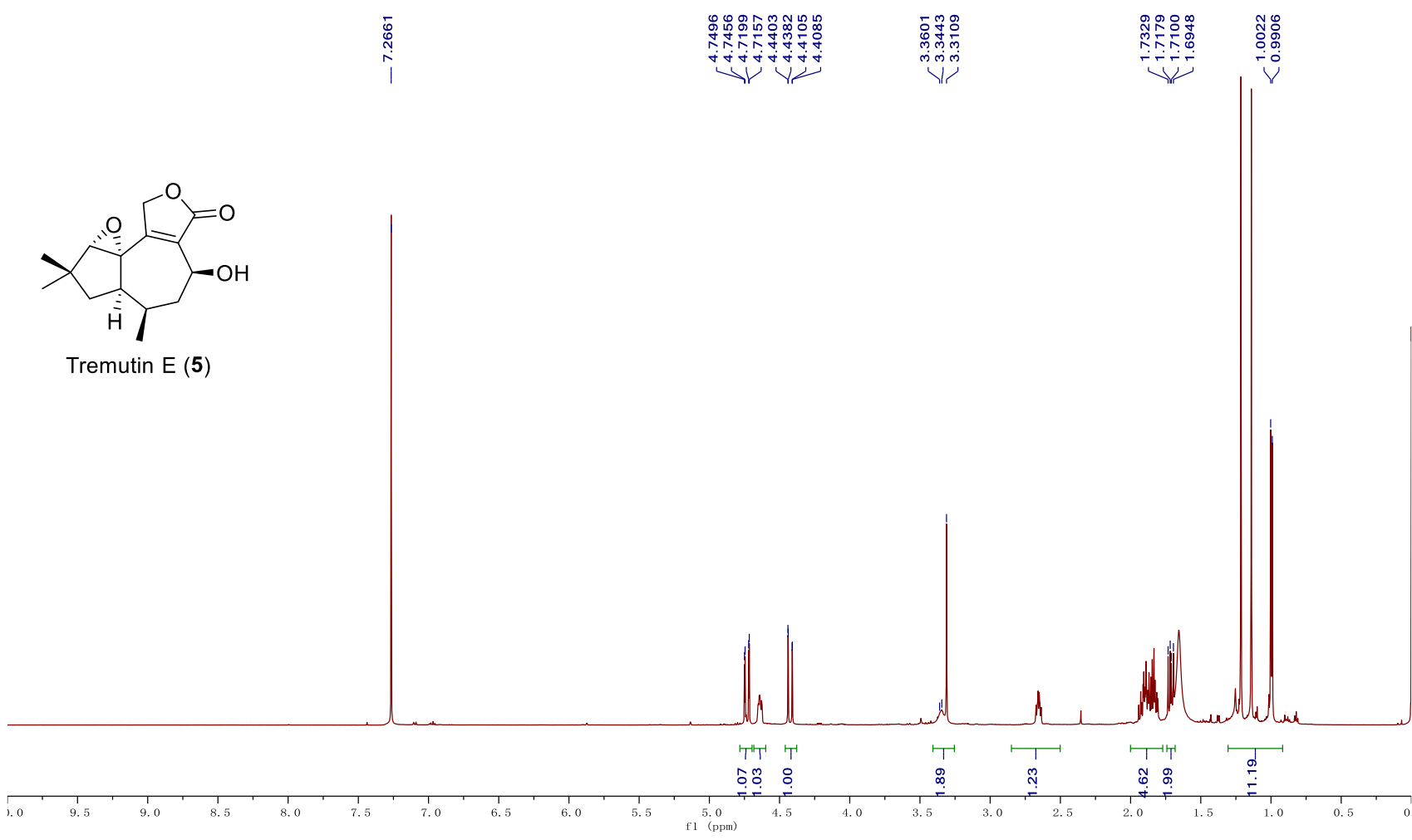

${ }^{13} \mathrm{C}$ NMR and DEPT spectra of 5
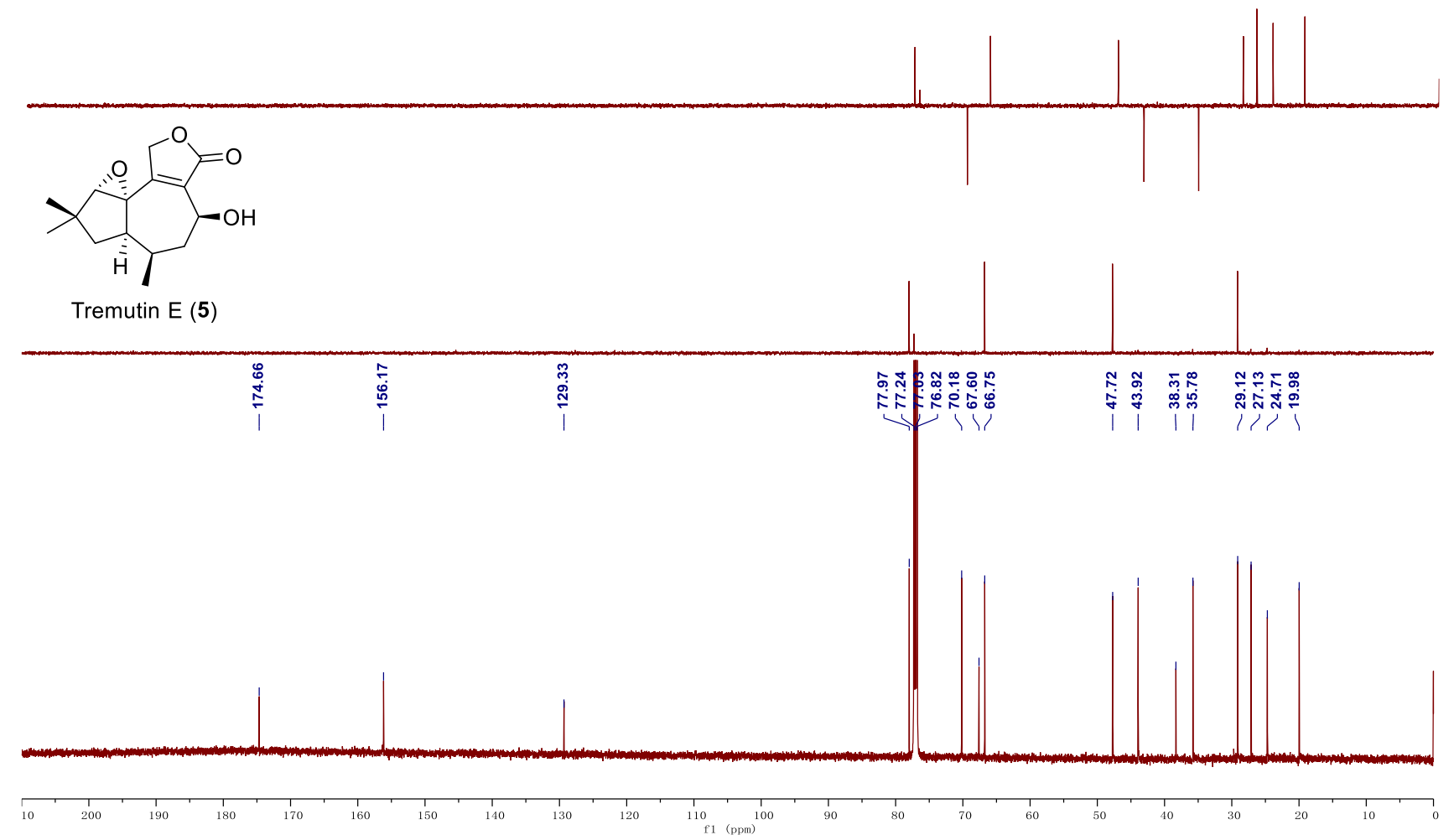


\section{HSQC spectrum of $\mathbf{5}$}
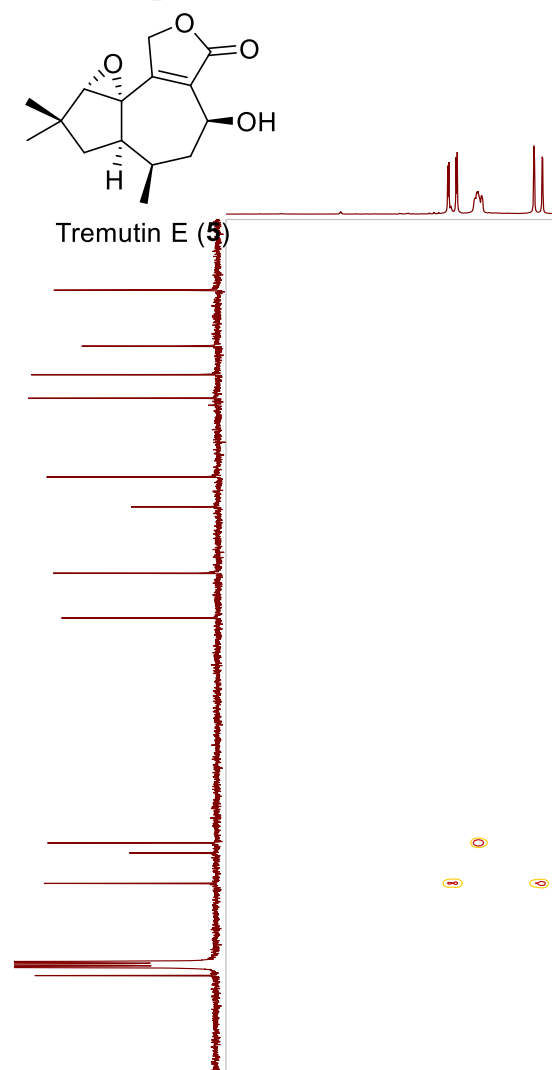

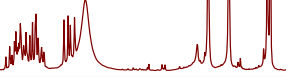

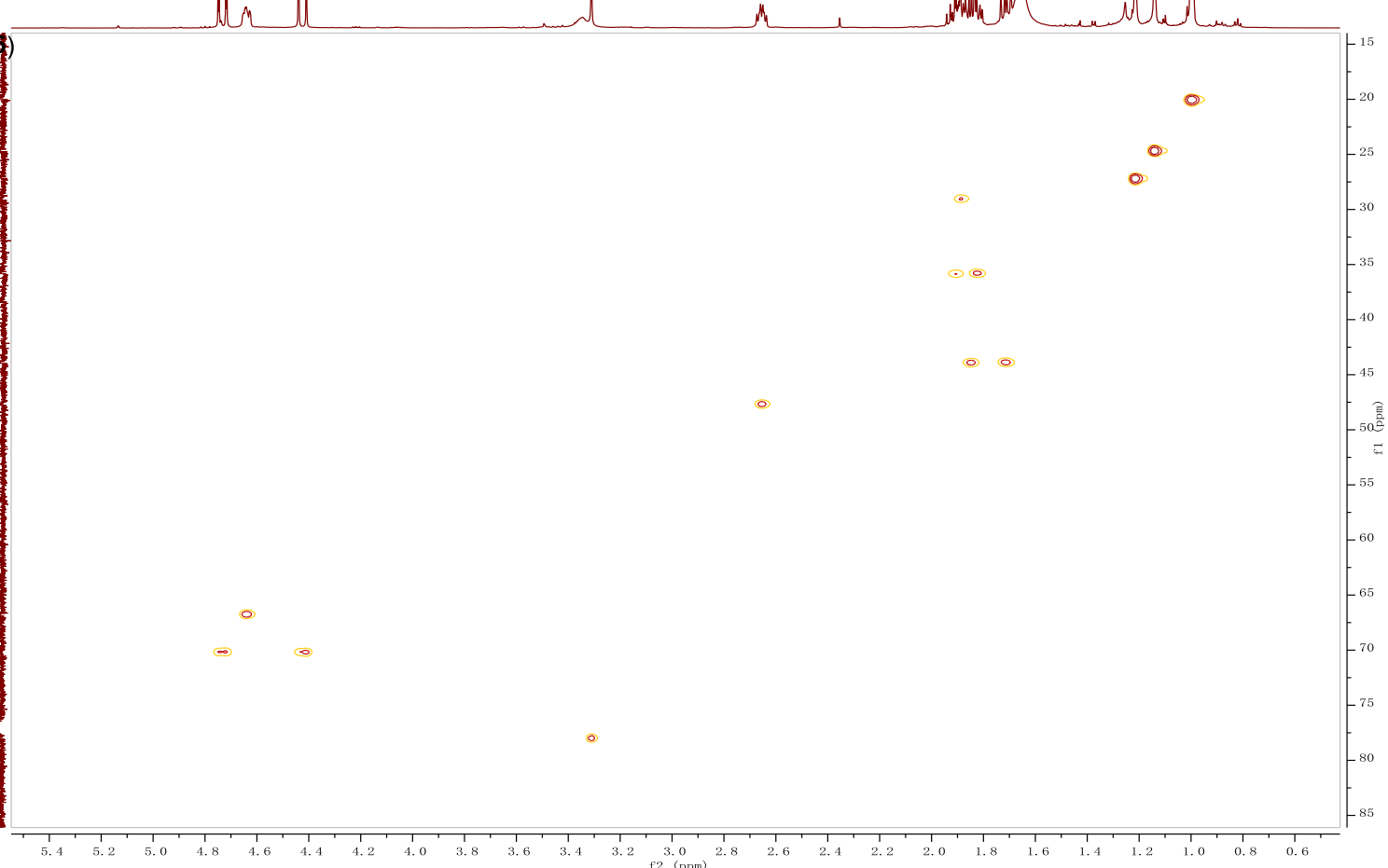

HMBC spectrum of 5
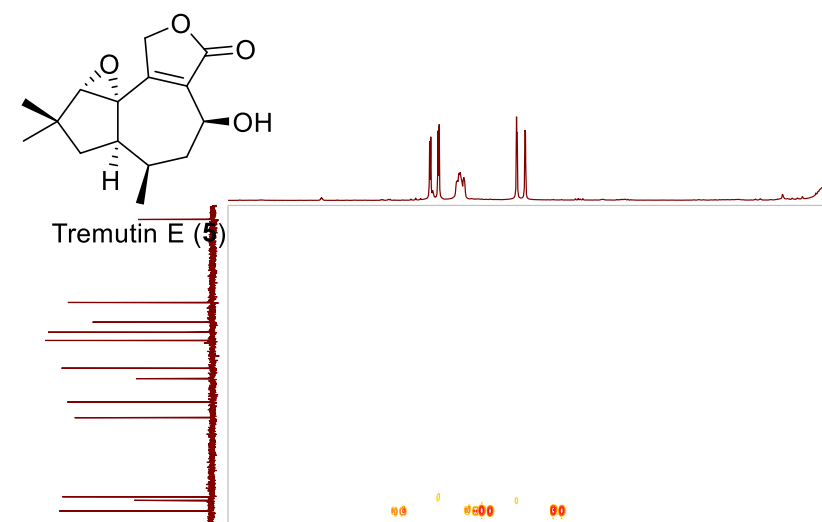
$\mu$
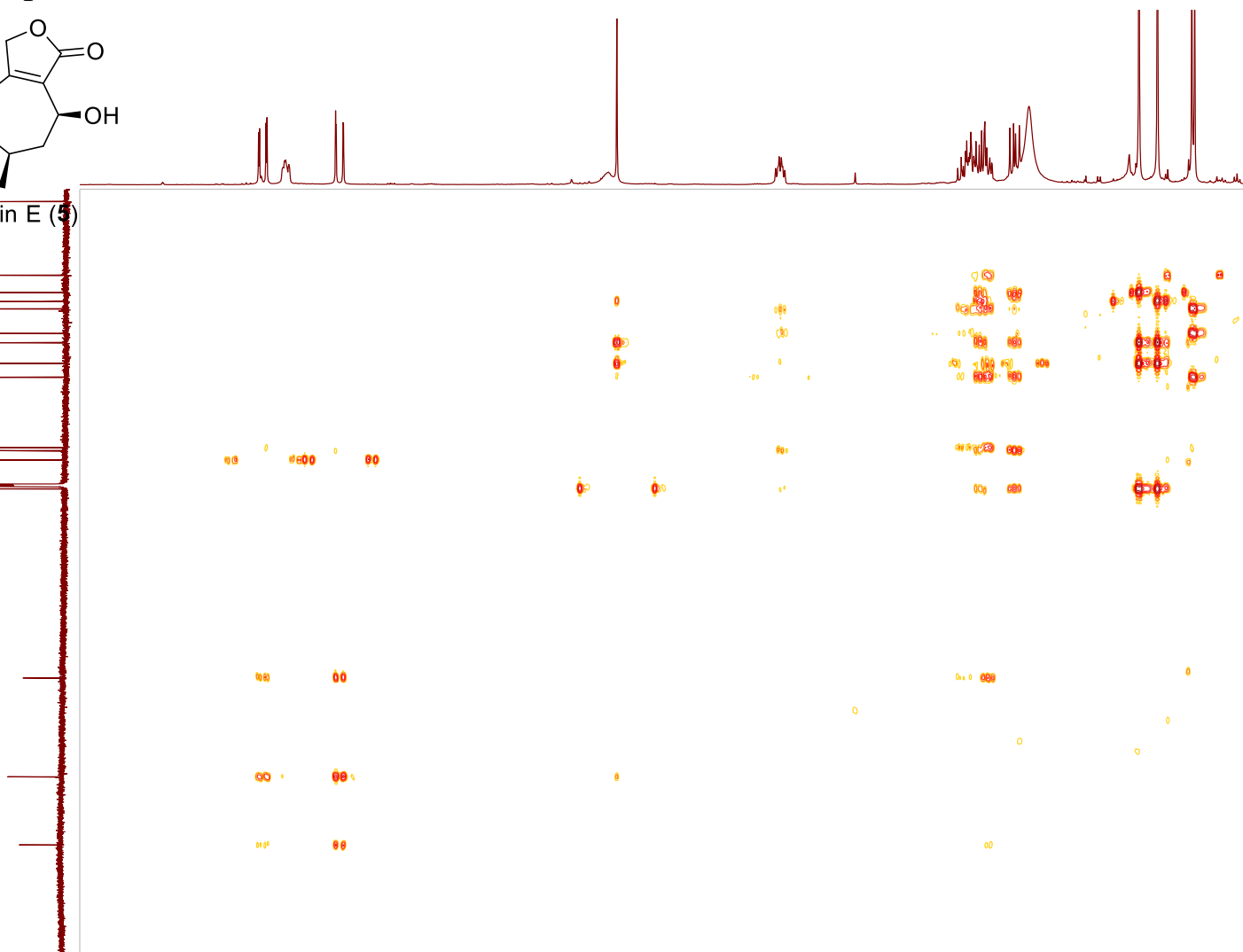
${ }^{1} \mathrm{H}-{ }^{1} \mathrm{H}$ COSY spectrum of 5

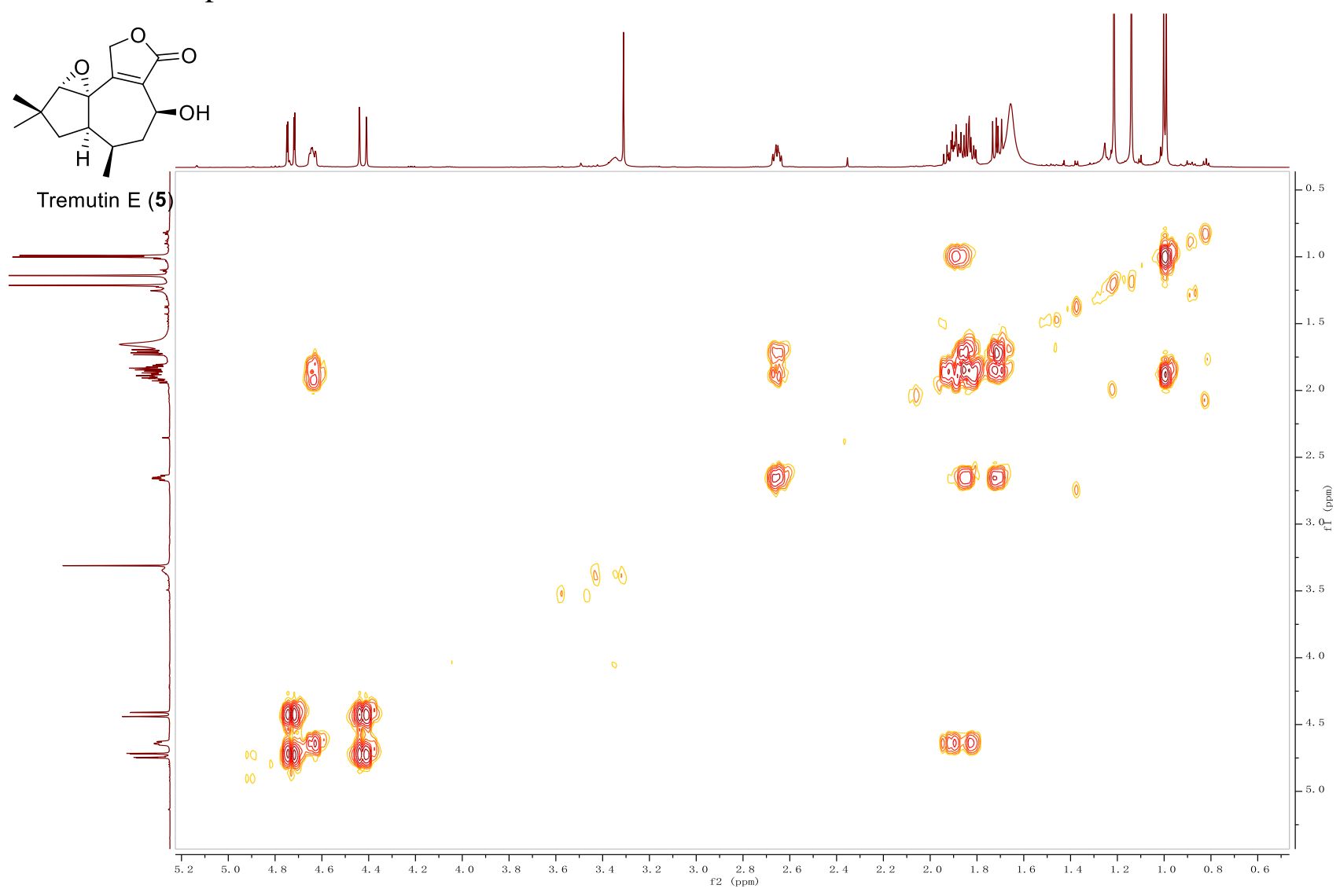

ROESY spectrum of 5

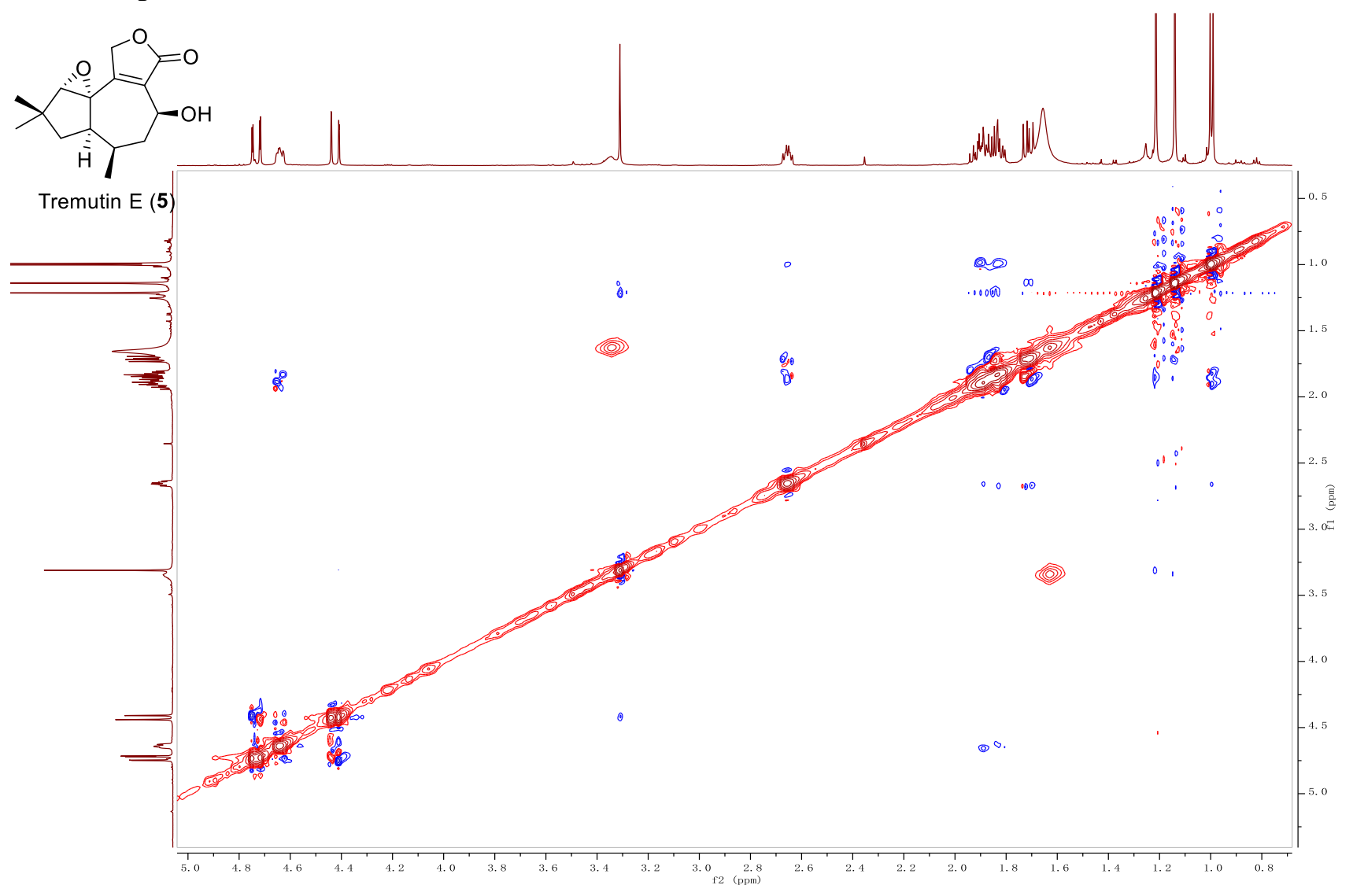




\section{HRESIMS of 5}

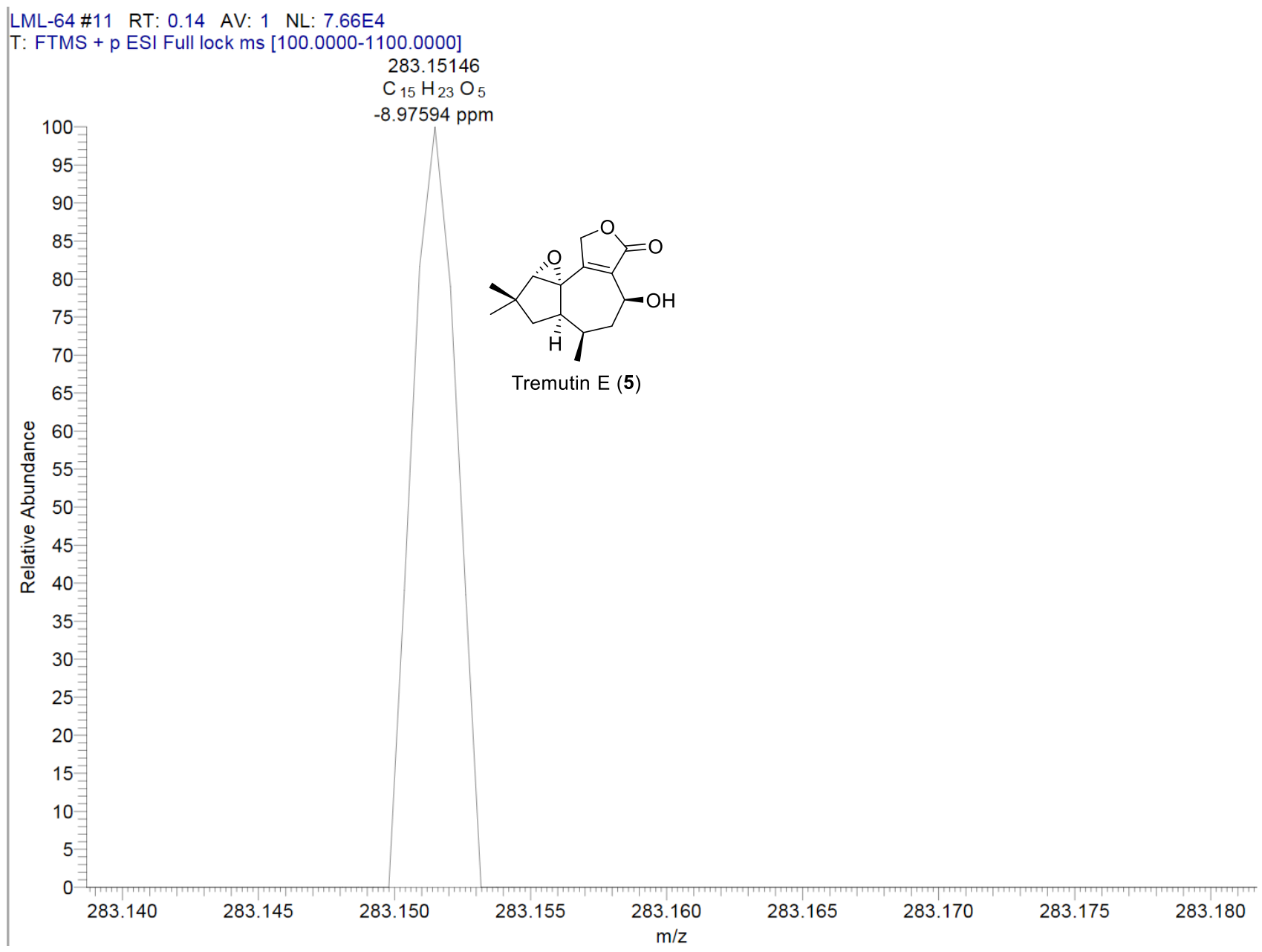


4.6 NMR, HRESIMS, and CD spectra for tremutin F (6)

${ }^{1} \mathrm{H}$ NMR spectrum of 6

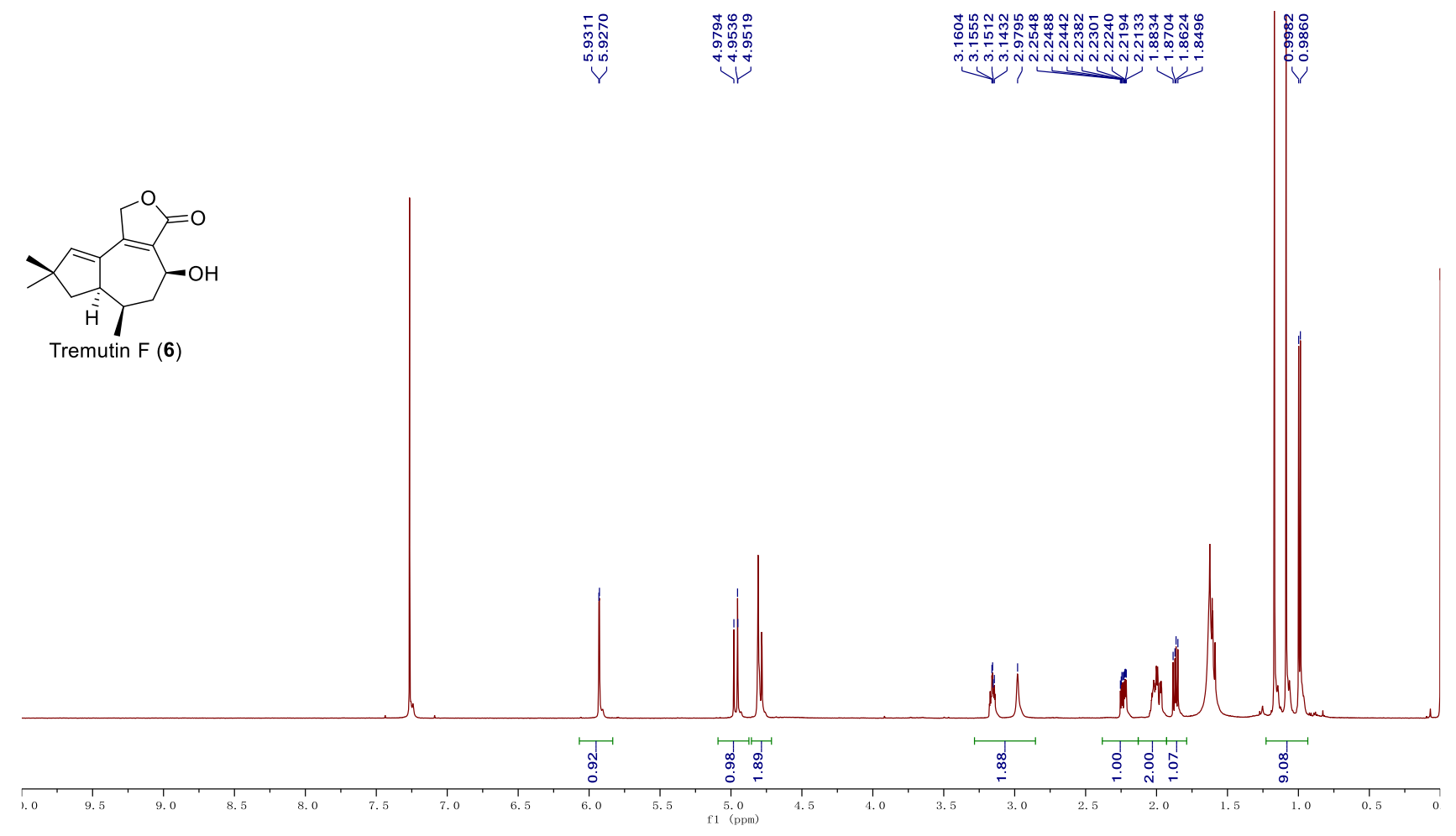

${ }^{13} \mathrm{C}$ NMR and DEPT spectra of 6
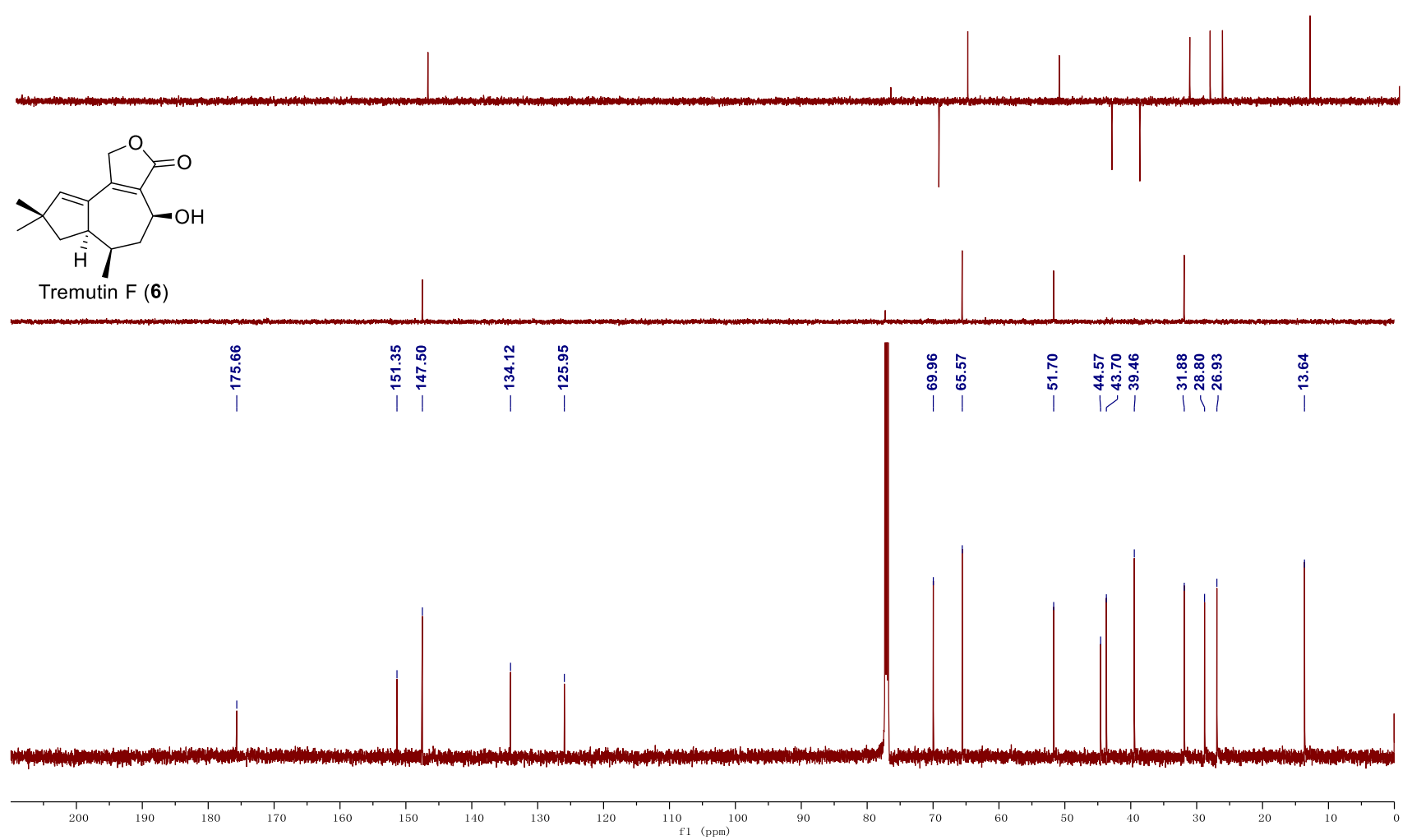


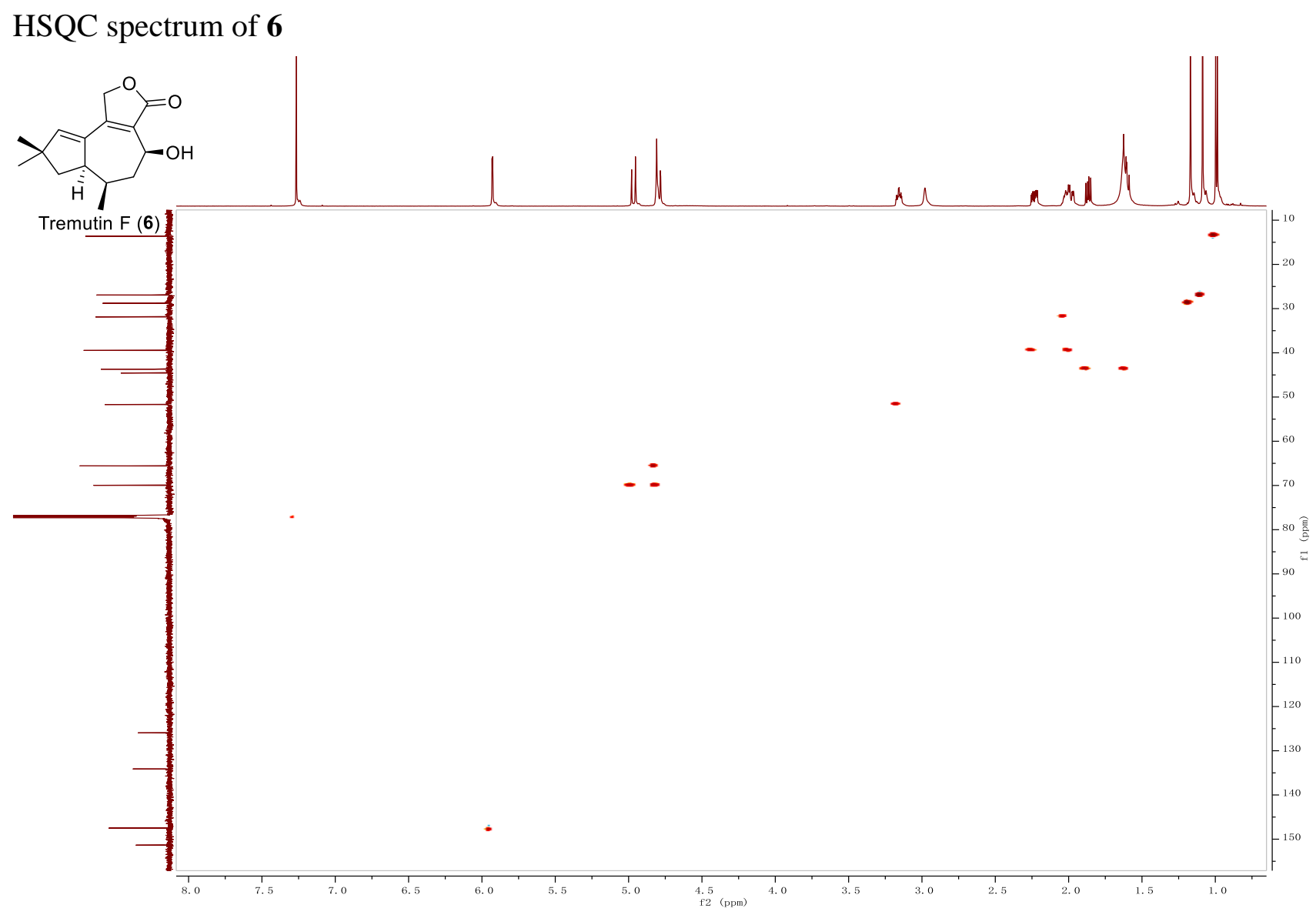

HMBC spectrum of 6

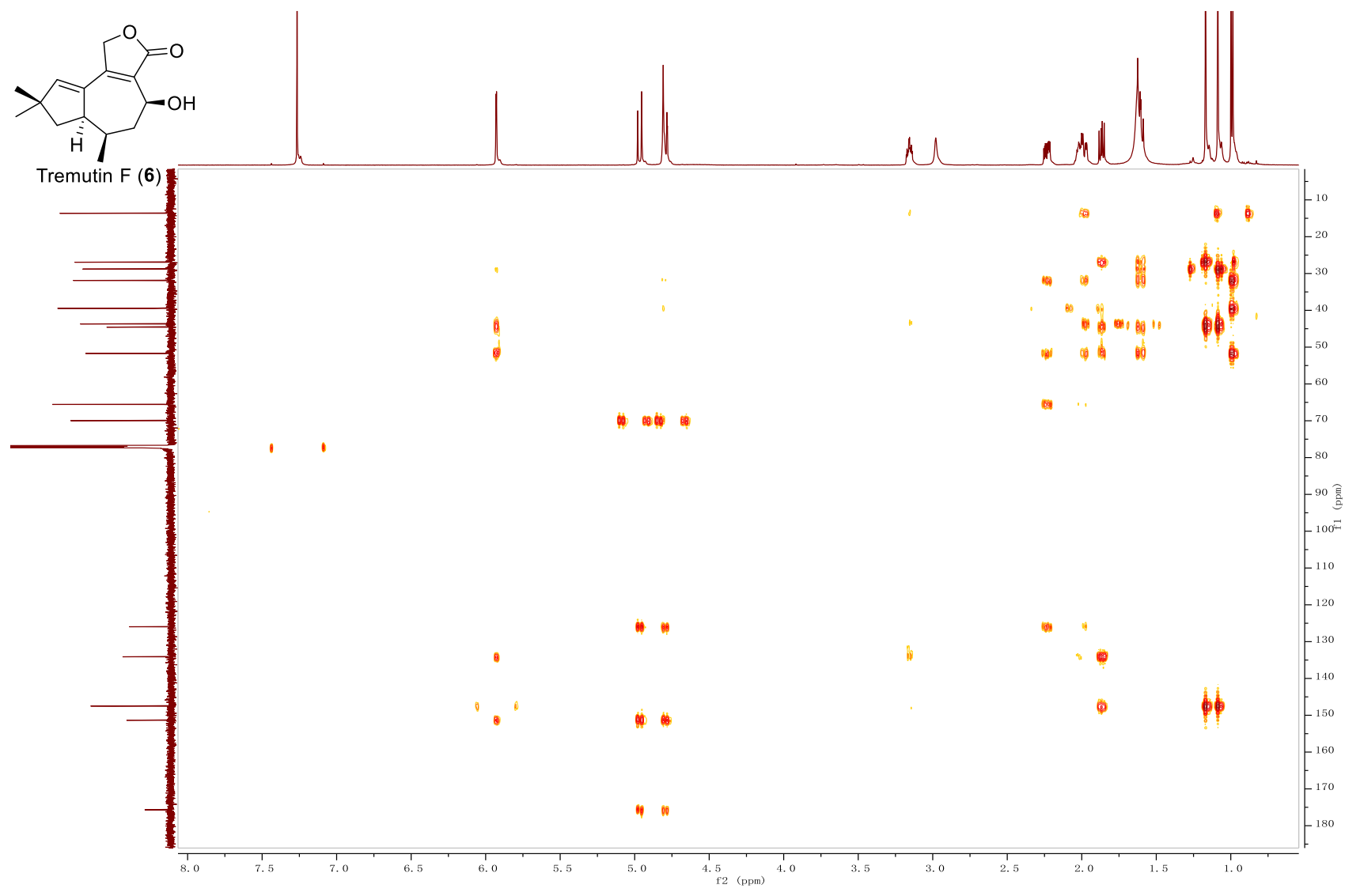


${ }^{1} \mathrm{H}-{ }^{1} \mathrm{H}$ COSY spectrum of 6

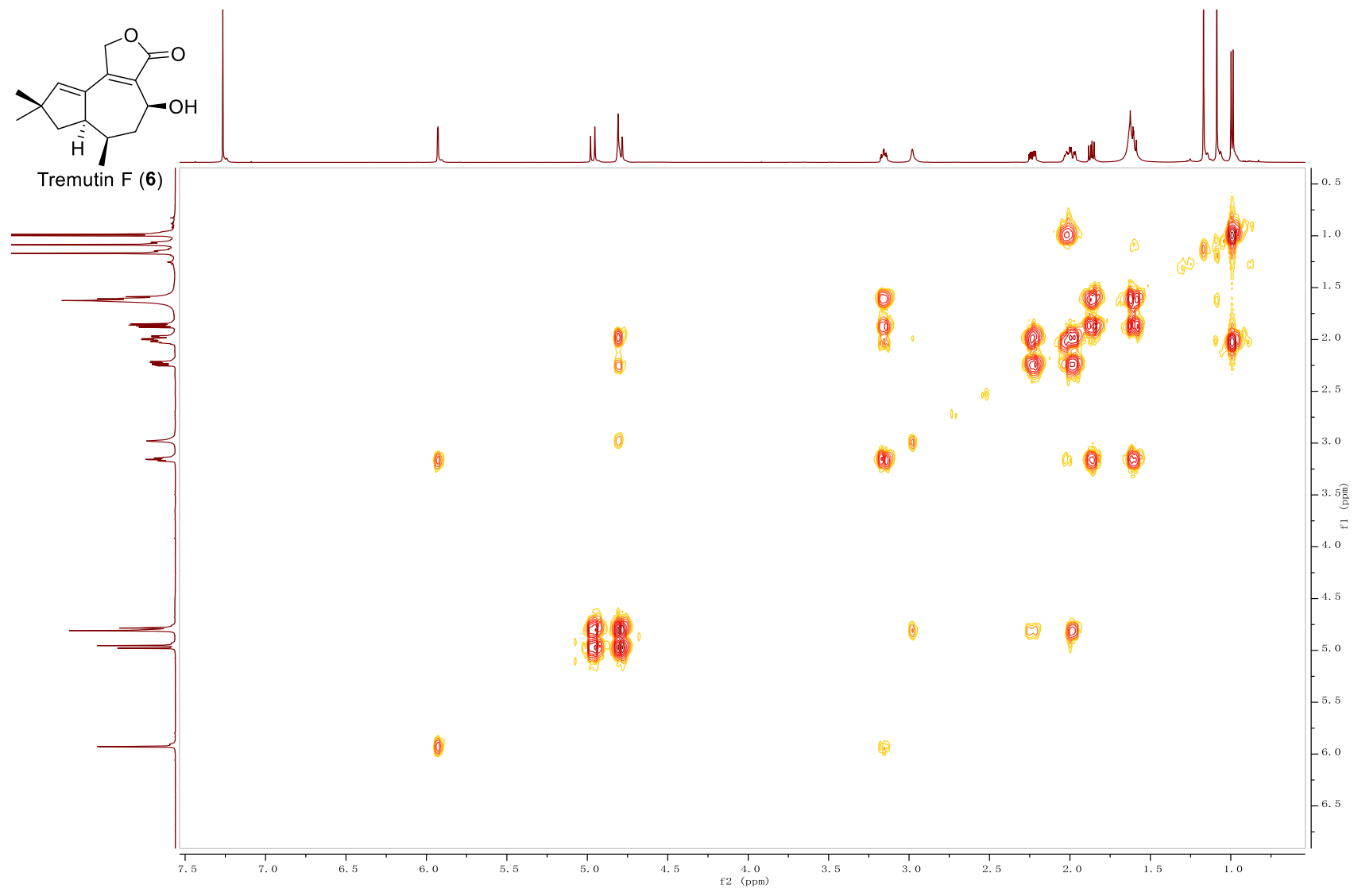

ROESY spectrum of $\mathbf{6}$

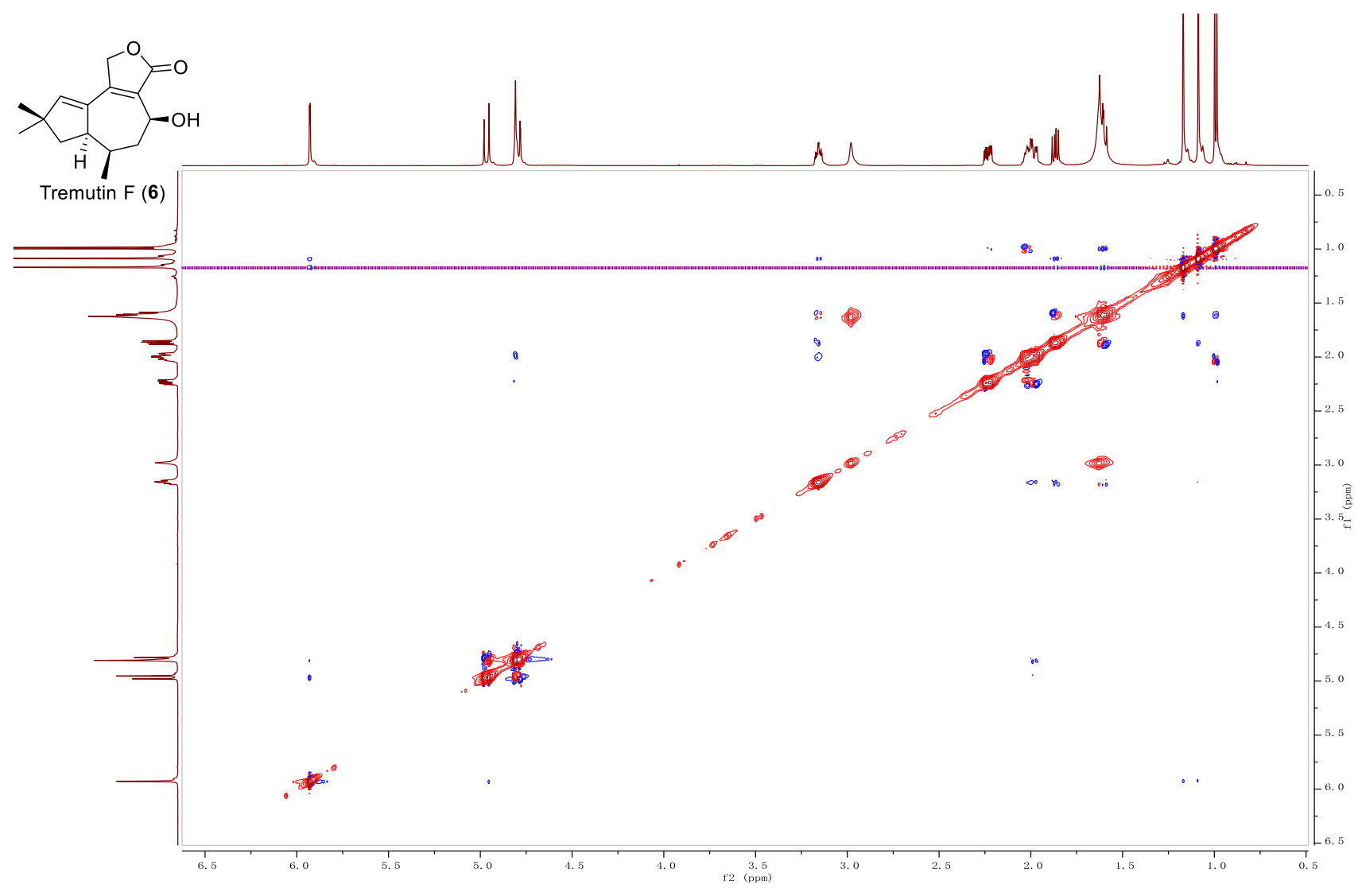




\section{HRESIMS of 6}

LML-77 \#13 RT: $0.17 \quad$ AV: 1 NL: $1.86 E 6$

T: FTMS + p ESI Full lock ms [100.0000-1100.0000]

249.14848

$100_{-}^{-0.15153 \mathrm{ppm}}$

95

90

85

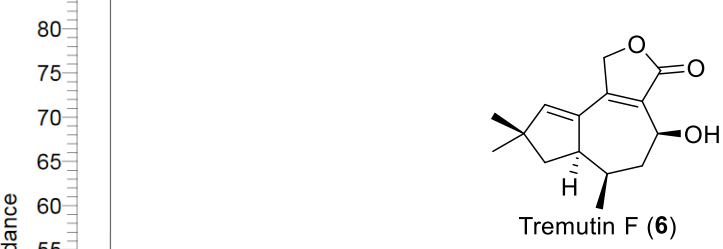

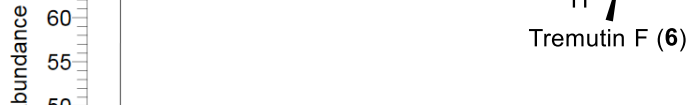

选 50

$\sum_{\frac{1}{D}}^{\infty} 45$

$\stackrel{\frac{\pi}{\mathbb{N}}}{\mathbb{1}} 40$

35

25

20

15

10

$0^{-3}$

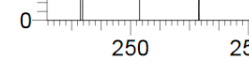

\section{CD spectrum of $\mathbf{6}$}

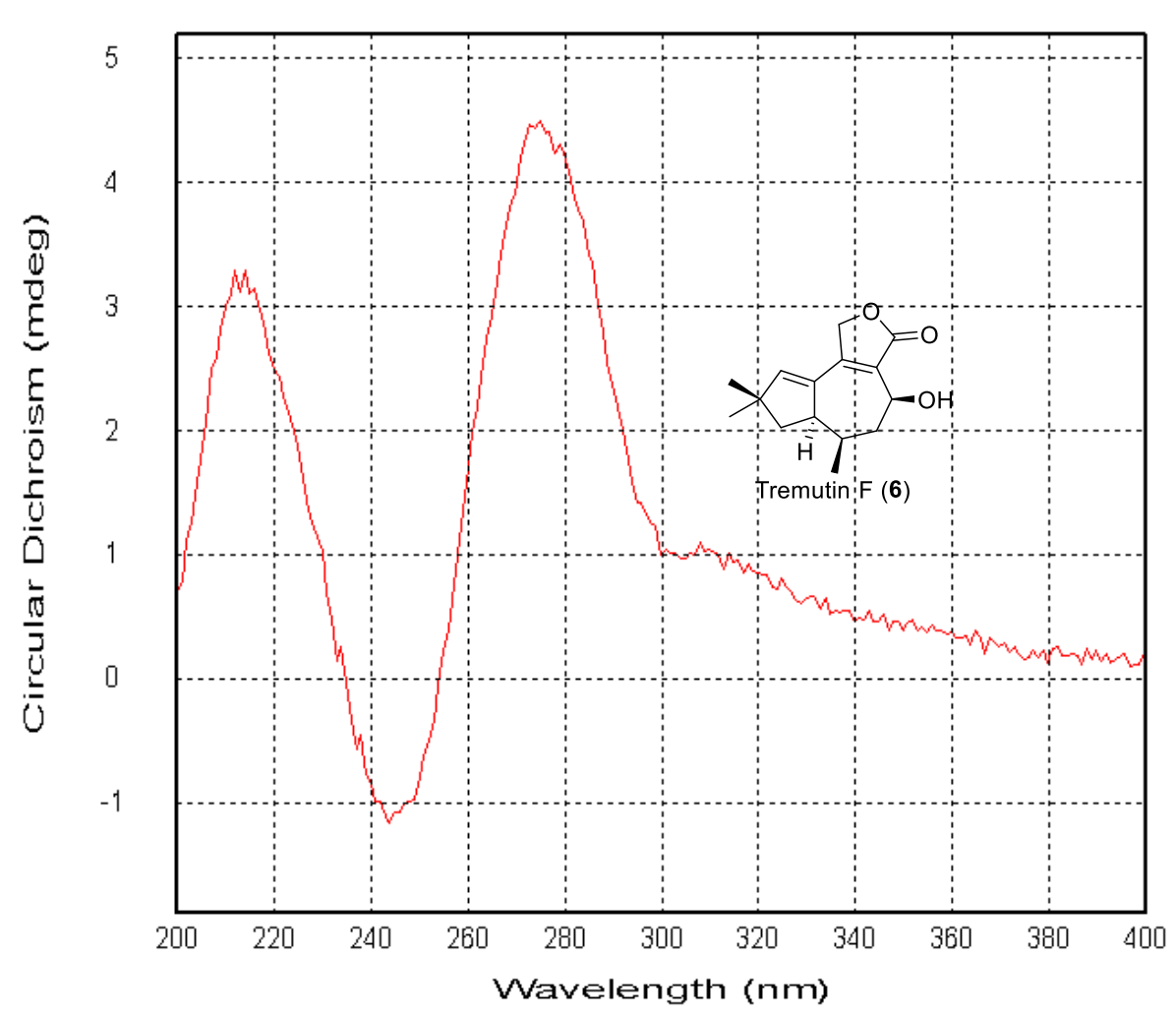

Subtracted:0 
4.7 NMR, HRESIMS, and CD spectra for tremutin G (7)

${ }^{1} \mathrm{H}$ NMR spectrum of 7
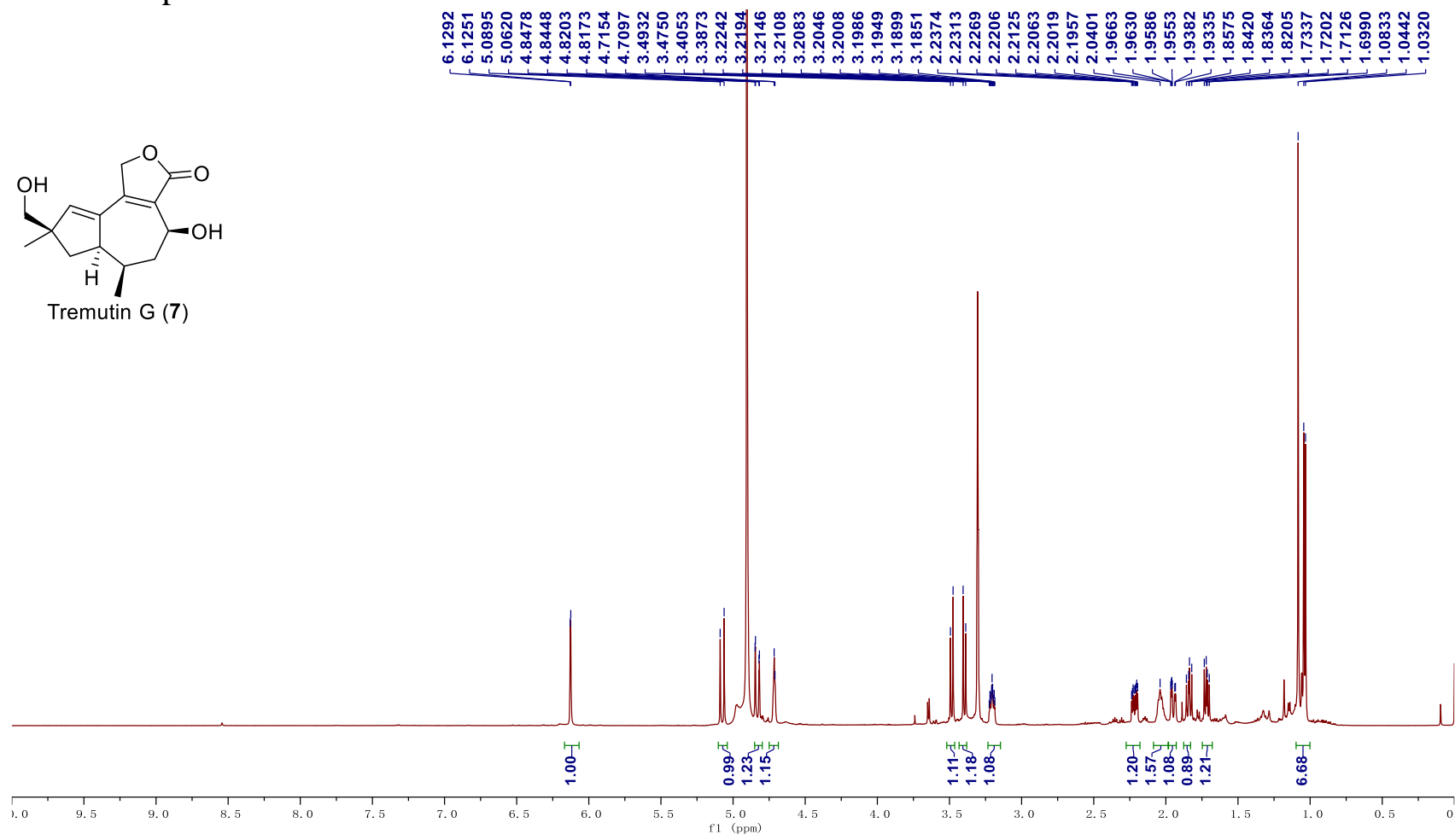

${ }^{13} \mathrm{C}$ NMR and DEPT spectra of 7

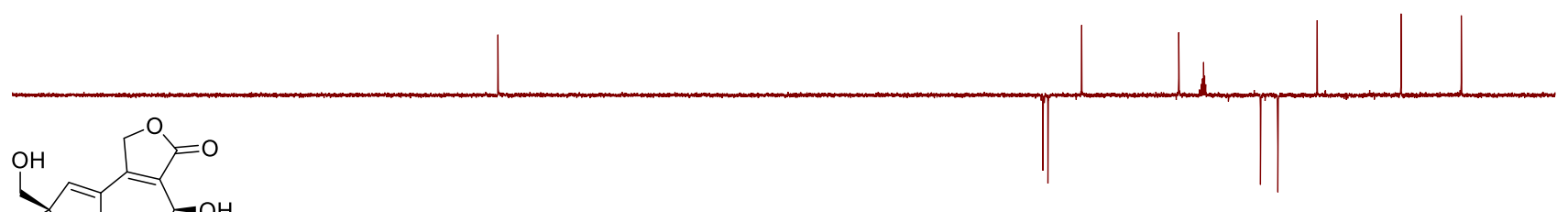

ì

Tremutin G (7)

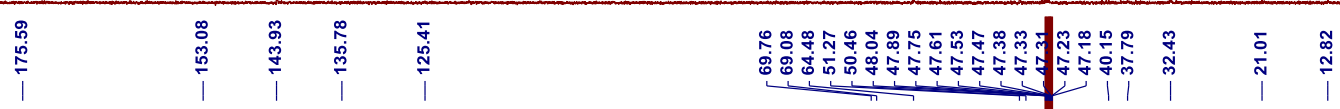

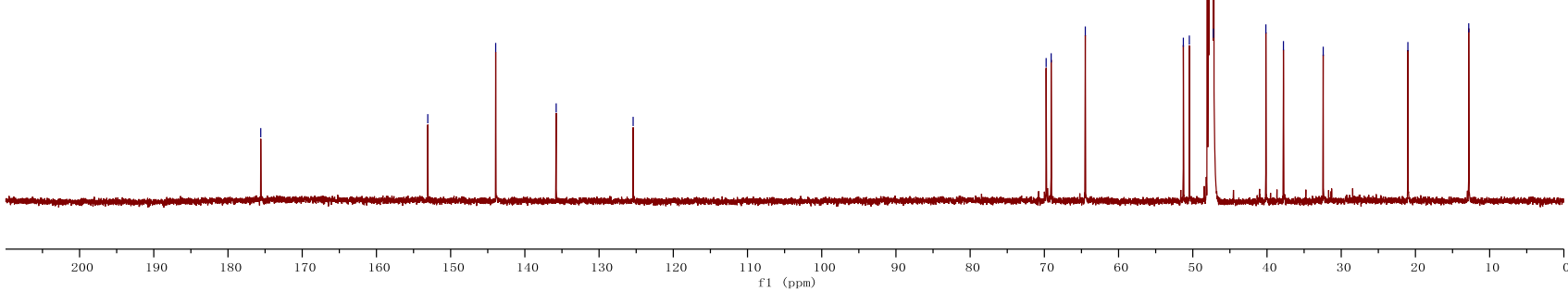


HSQC spectrum of 7

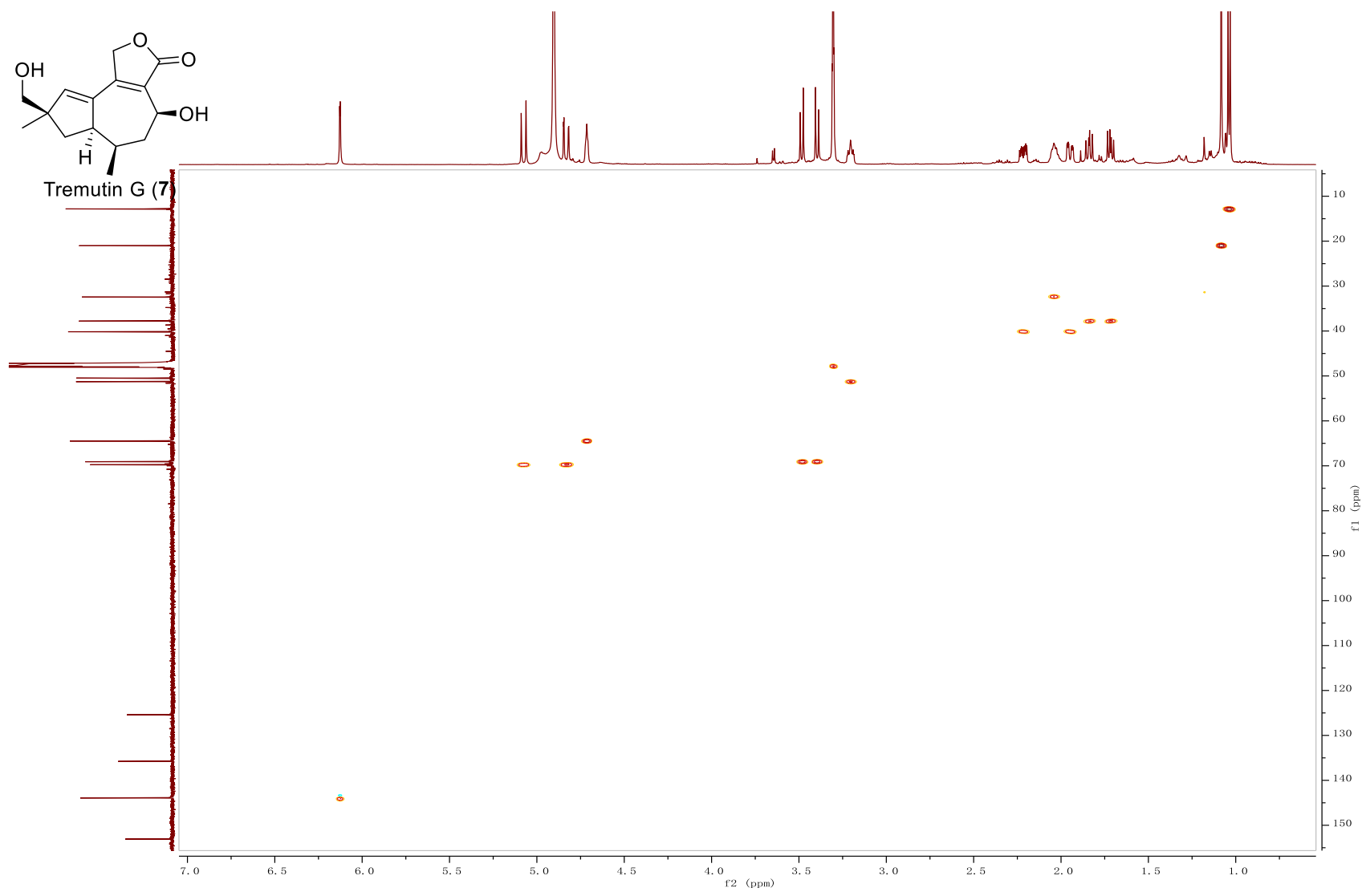

HMBC spectrum of 7

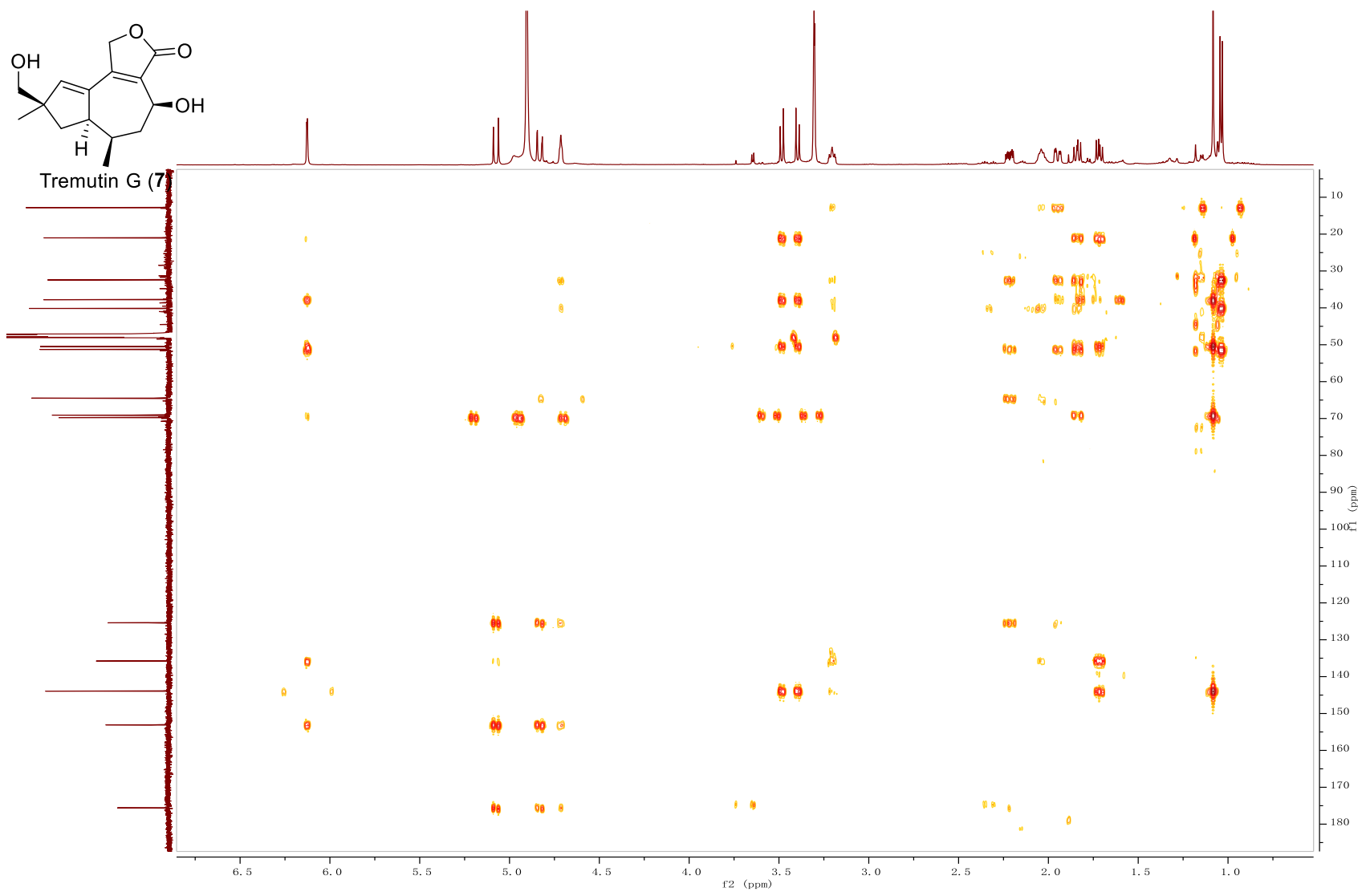


${ }^{1} \mathrm{H}-{ }^{1} \mathrm{H}$ COSY spectrum of 7

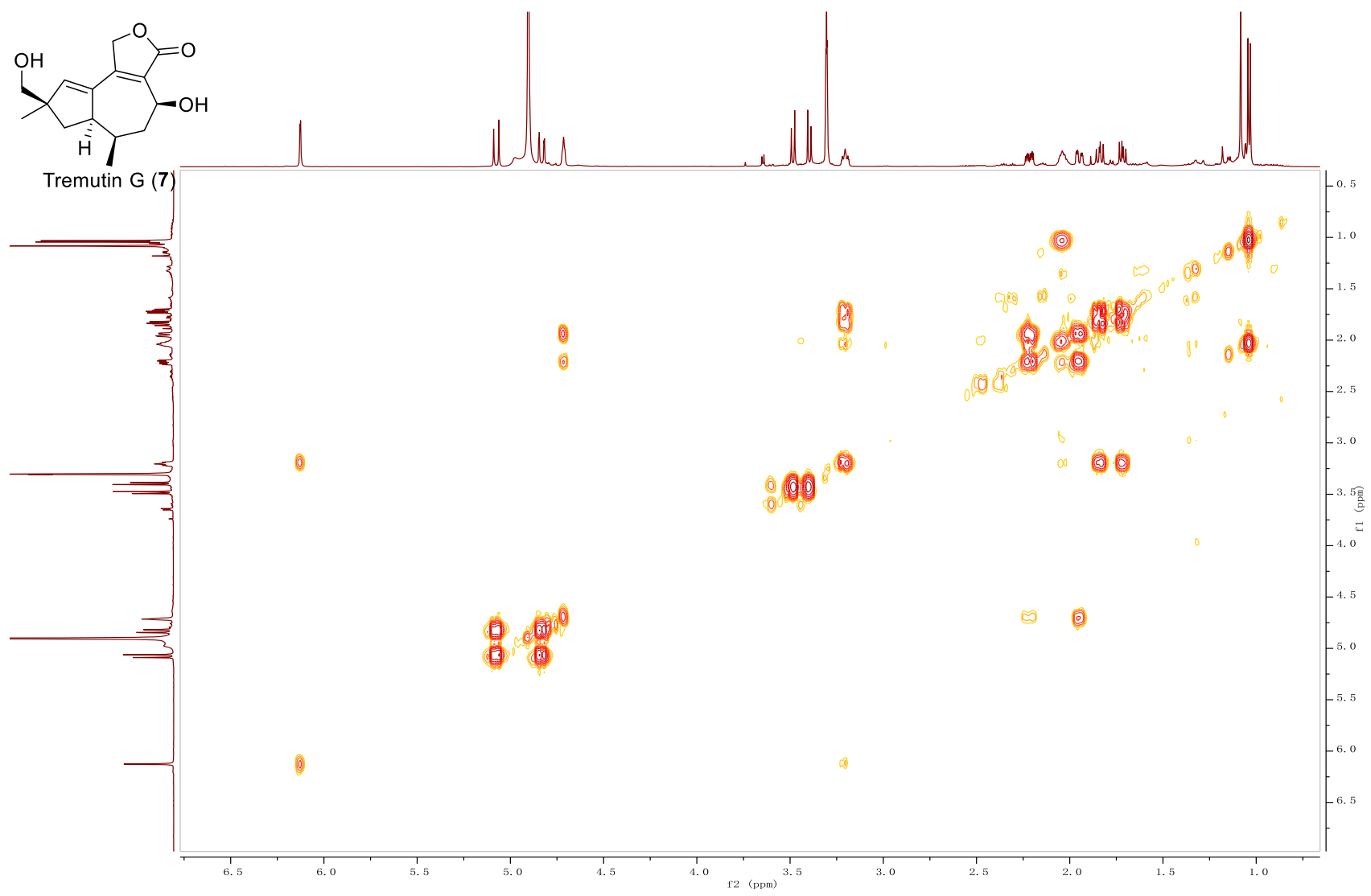

ROESY spectrum of 7

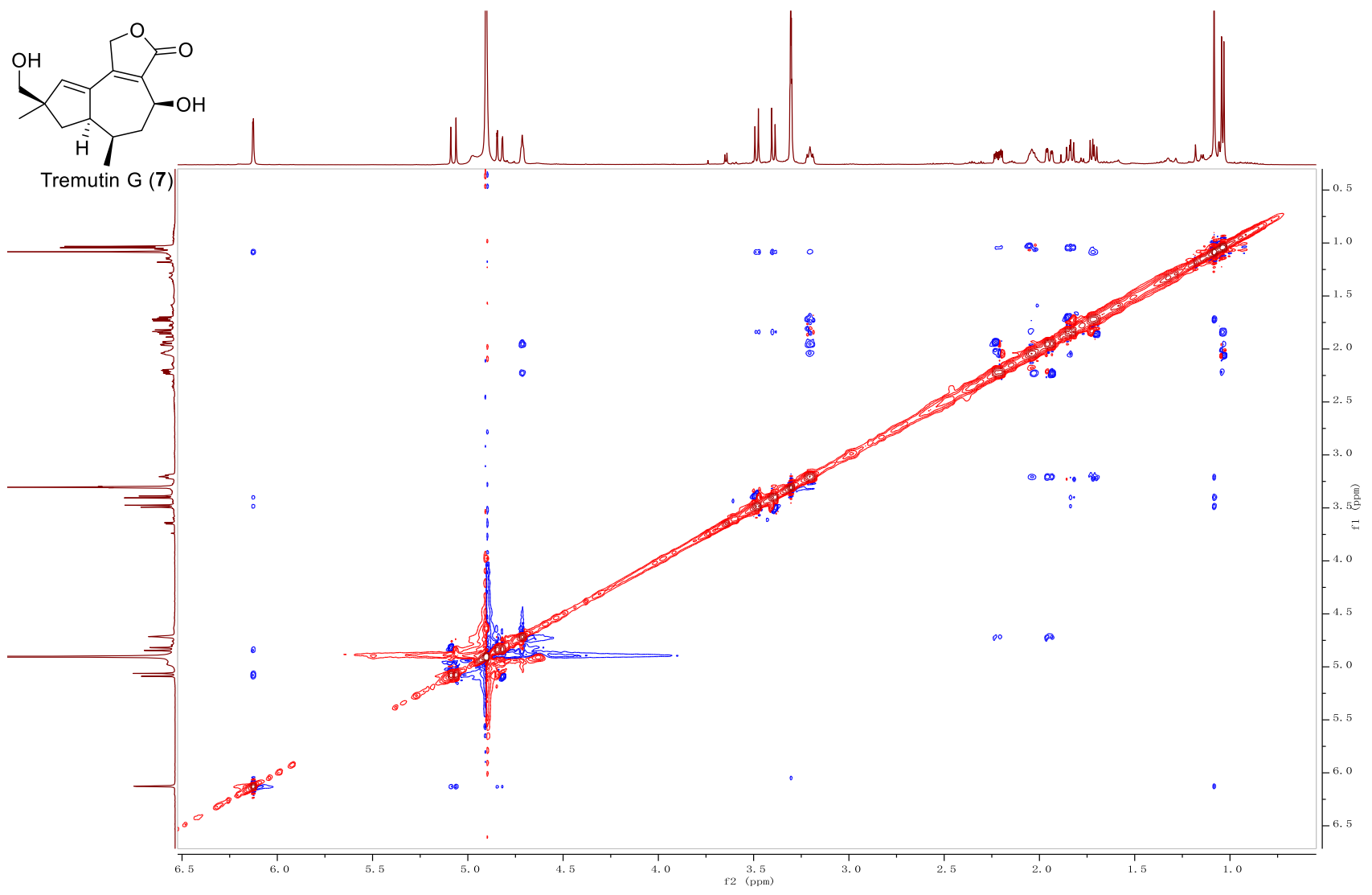




\section{HRESIMS of 7}

LML-176 \#13 RT: 0.17 AV: 1 NL: $1.37 E 8$

T: FTMS + p ESI Full lock ms [150.0000-1100.0000]

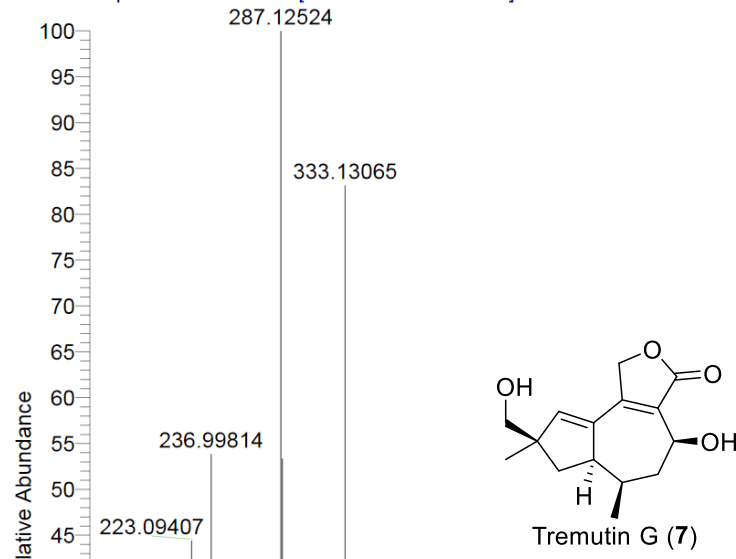

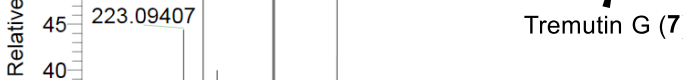

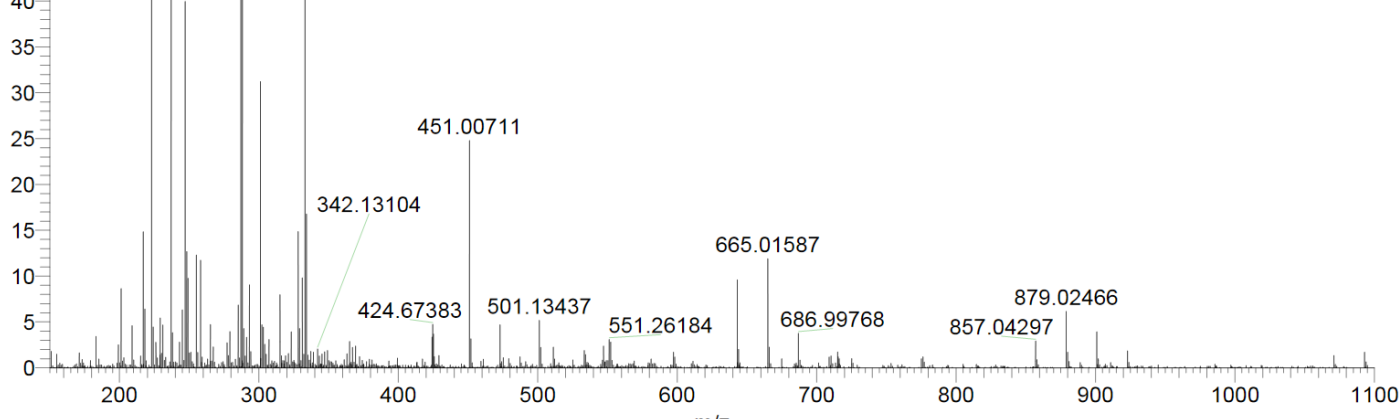

CD spectrum of 7

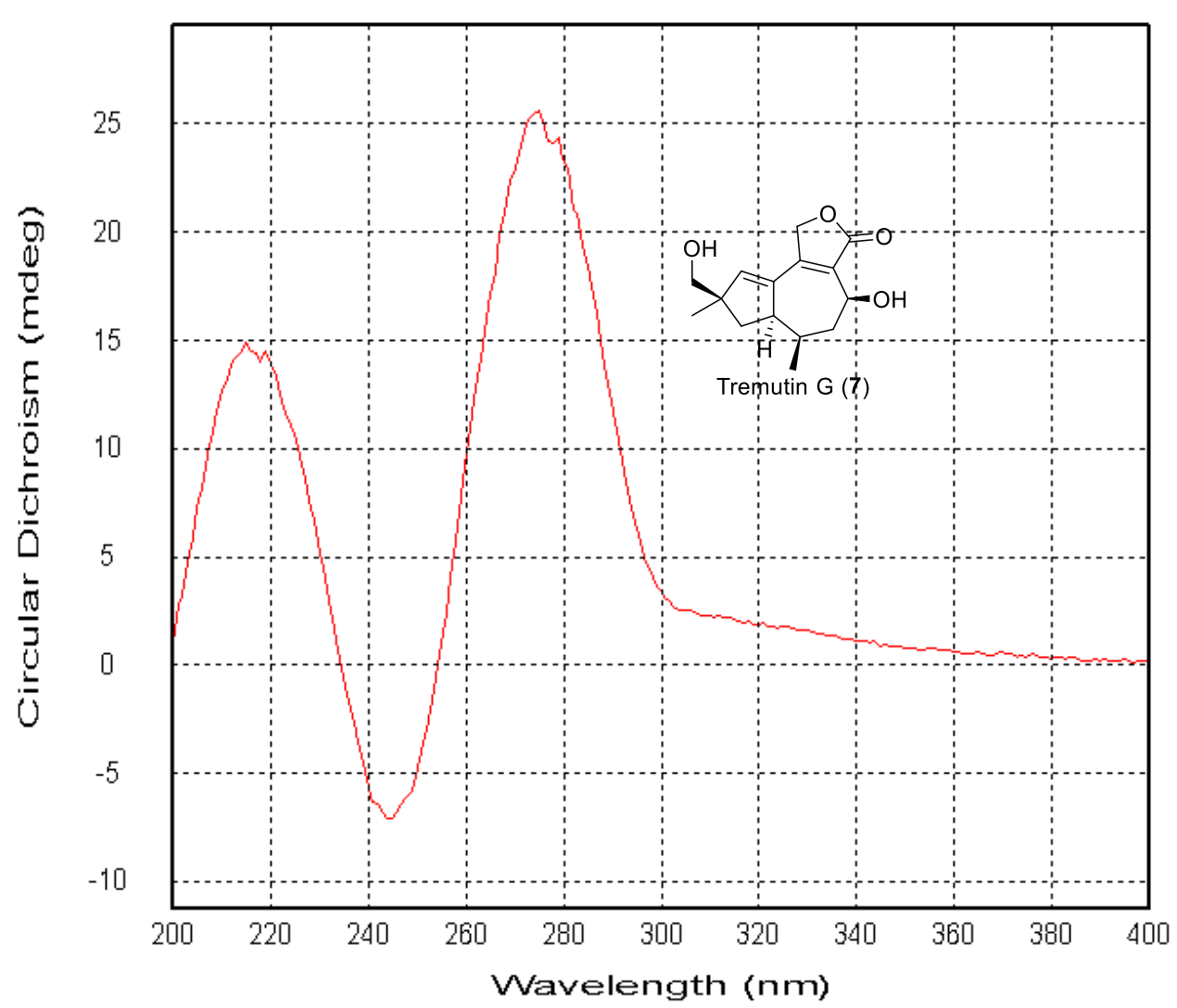

Subtracted:0 
4.8 NMR and HRESIMS for tremutin H (8)

${ }^{1} \mathrm{H}$ NMR spectrum of $\mathbf{8}$

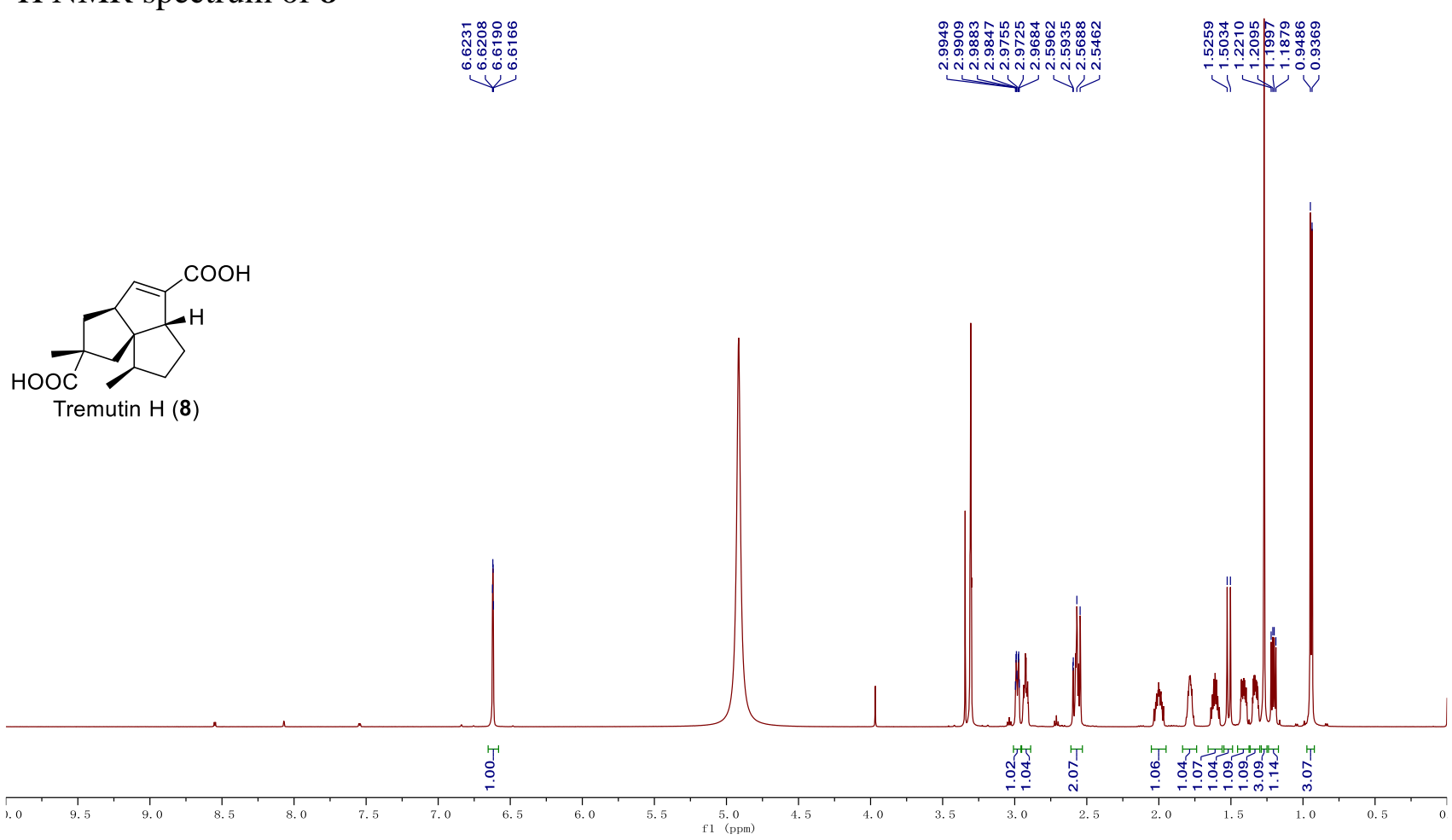

${ }^{13} \mathrm{C}$ NMR and DEPT spectra of $\mathbf{8}$
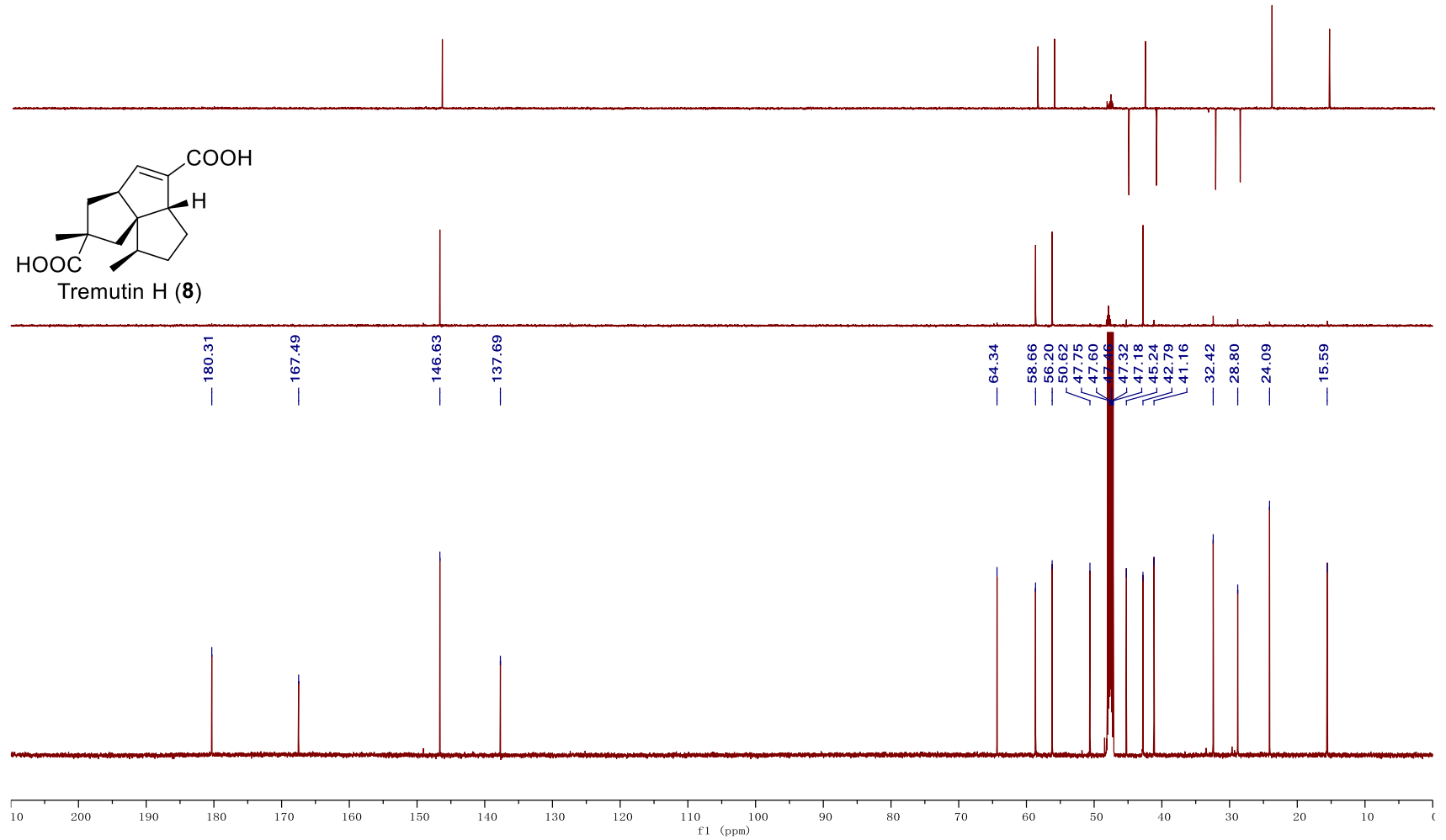
HSQC spectrum of $\mathbf{8}$

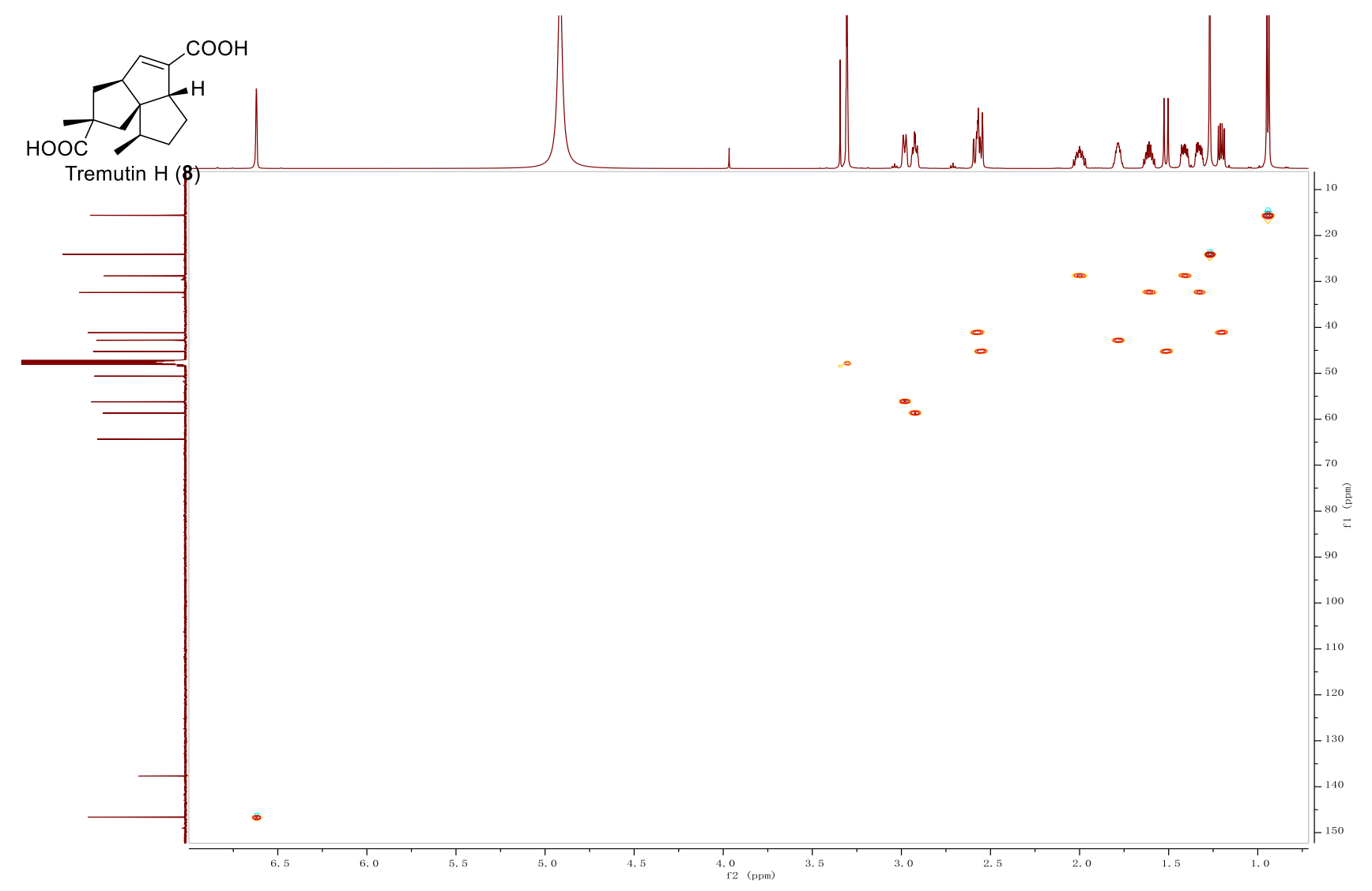

HMBC spectrum of 8

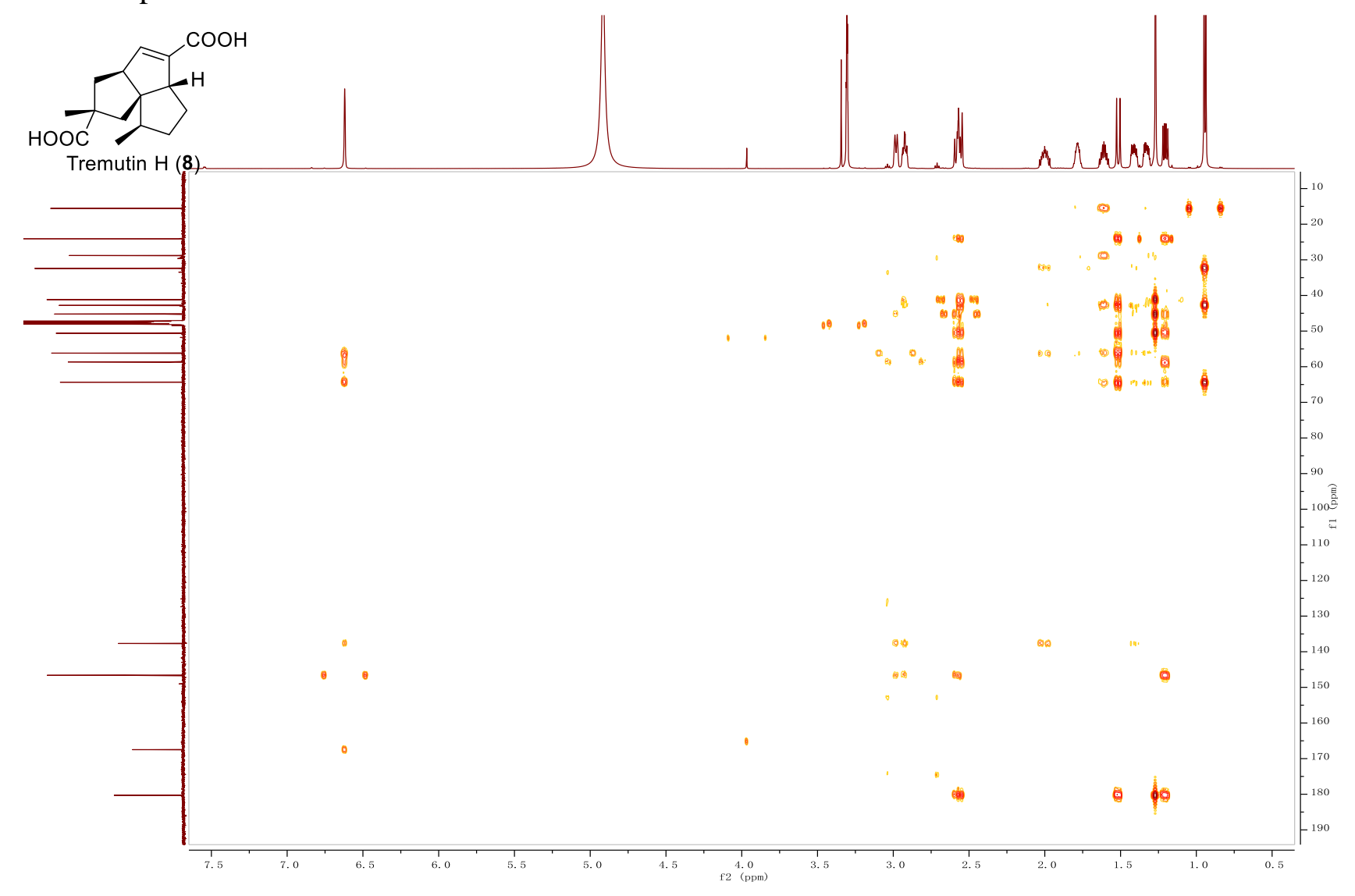




\section{${ }^{1} \mathrm{H}-{ }^{1} \mathrm{H}$ COSY spectrum of 8}

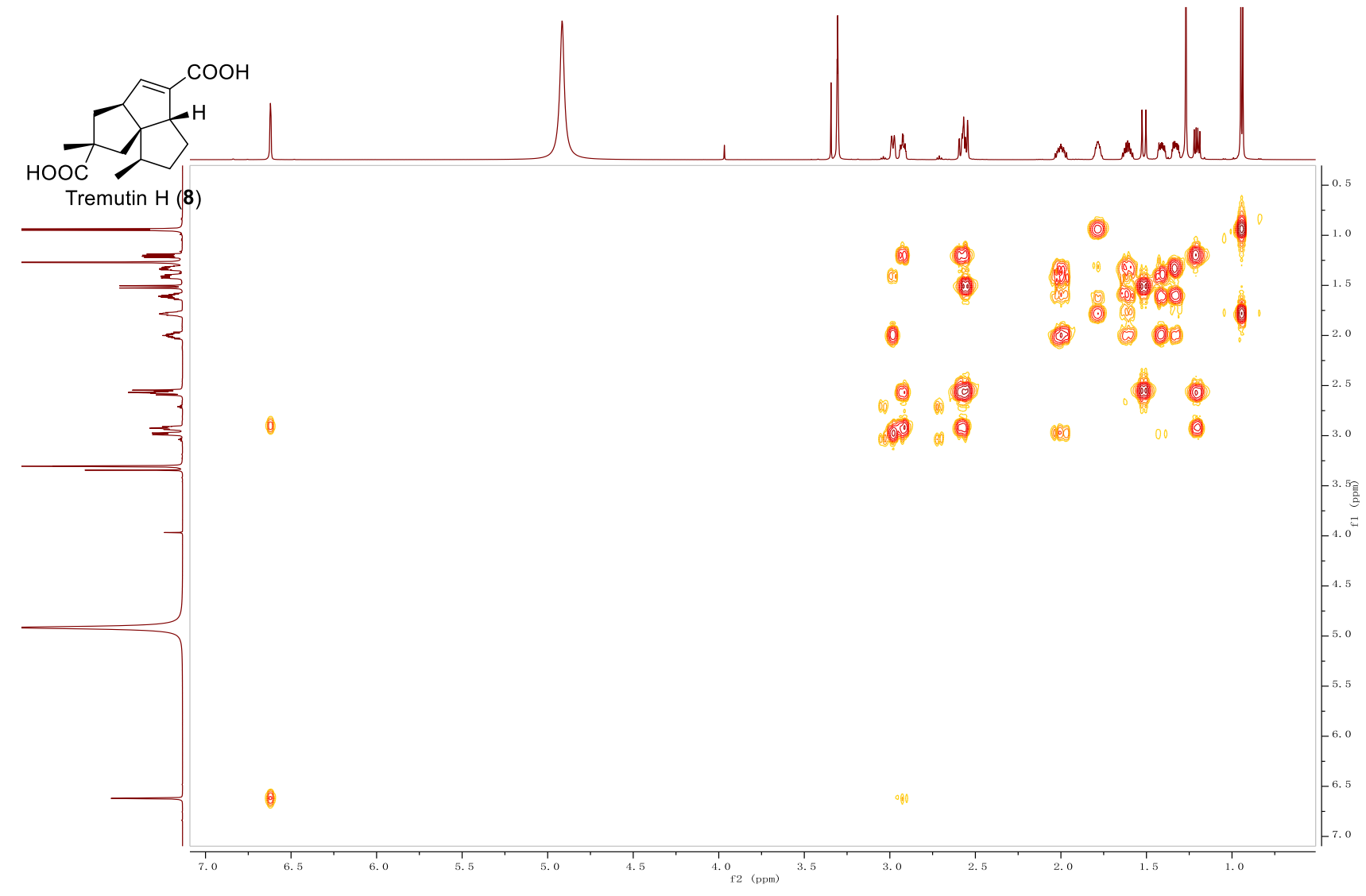

\section{HRESIMS of 8}

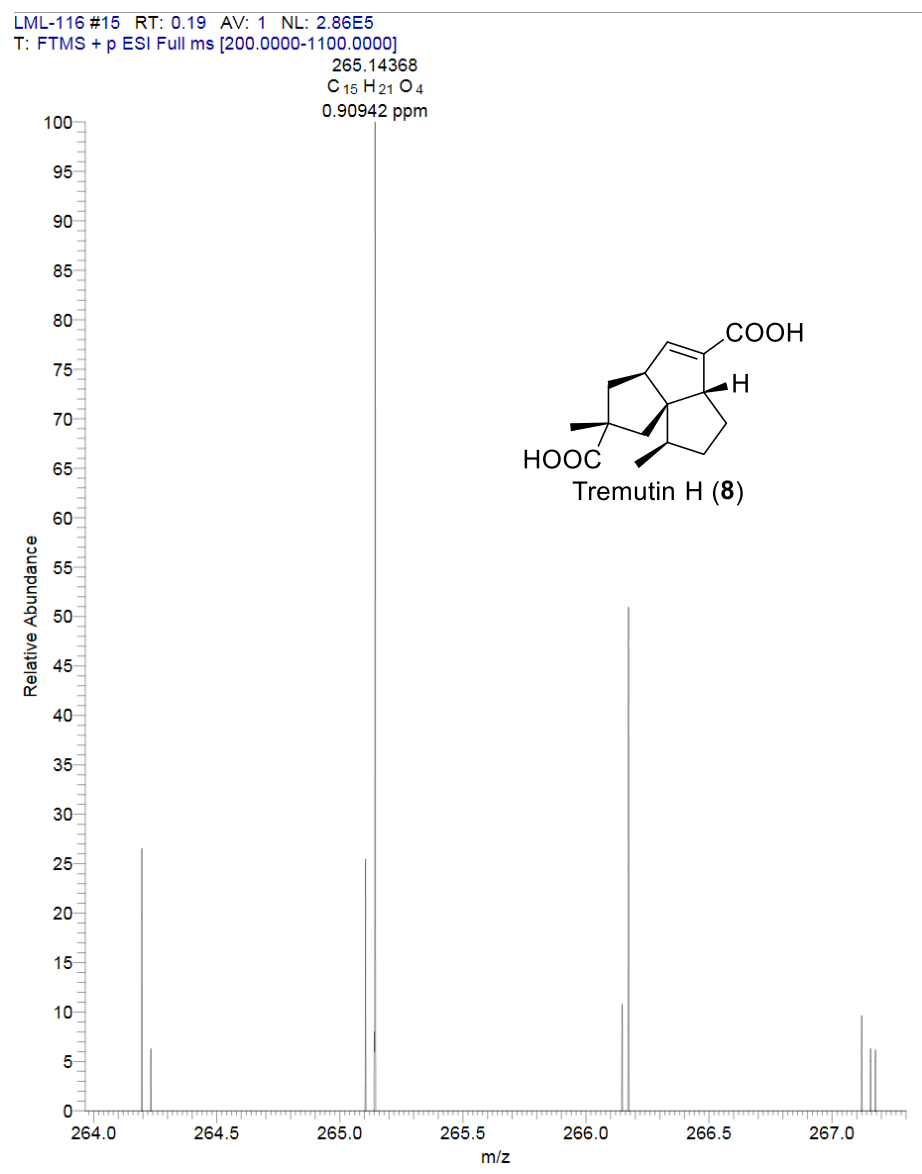

